\title{
On the Structure of Unitary Conformal Field Theory II: Representation Theoretic Approach
}

\author{
G. Felder, J. Fröhlich, and G. Keller \\ Theoretische Physik, ETH-Hönggerberg, CH-8093 Zürich, Switzerland
}

\begin{abstract}
Two-dimensional, unitary rational conformal field theory is studied from the point of view of the representation theory of chiral algebras. Chiral algebras are equipped with a family of co-multiplications which serve to define tensor product representations. Chiral vertices arise as Clebsch-Gordan operators from tensor product representations to irreducible subrepresentations of a chiral algebra. The algebra of chiral vertices is studied and shown to give rise to representations of the braid groups determined by Yang-Baxter (braid) matrices. Chiral fusion is analyzed. It is shown that the braid- and fusion matrices determine invariants of knots and links. Connections between the representation theories of chiral algebras and of quantum groups are sketched. Finally, it is shown how the local fields of a conformal field theory can be reconstructed from the chiral vertices of two chiral algebras.
\end{abstract}

\section{Introduction}

In their seminal paper [19], Belavin, Polyakov and Zamolodchikov pointed out that the existence of infinite-dimensional symmetries in two dimensional conformal field theory has far-reaching consequences. In subsequent developments Friedan, Qiu and Shenker, and Goddard, Kent and Olive, [20] proved crucial results on the representation theory of Virasoro algebras. These can be applied to yield a partial classification of all unitary conformal field theories on $S^{2}$ with central charge $c<1$. More precisely, Ref. [20] determines the permissible set of conformal dimensions and the values of $c$, but not the operator algebras. In an attempt at a classification of the $c>1$ theories, various groups [21] have proposed some discrete series related to chiral algebras which are (supersymmetric) extensions of the Virasoro algebra. In this paper, we suggest a classification of all unitary, rational, local conformal field theories on the plane in terms of the representation theory of chiral algebras, and associated linear representations of the braid groups on $S^{2}$.

The present paper is the continuation of [1], where it was shown, that under suitable assumptions (which are typical for rational CFT) a given local, Möbius- 
covariant quantum field theory on the plane can be continued analytically to $\mathbb{C}^{2}$, that the field operators $\phi(z, \bar{z})$, decompose into sums of products of holomorphic chiral vertices, $\varphi(z)$, and of their antiholomorphic counterparts. Moreover, the chiral vertices obey a braid algebra when being continued analytically; i.e., the analytic continuation of the product $\varphi_{a}(z) \varphi_{b}(w)$ along a path, $\gamma$, exchanging $z$ and $w$ in positive orientation is given by $\mathscr{A}_{\gamma}\left(\varphi_{a}(z) \varphi_{b}(w)\right)=\sum_{c, d} R_{a b}^{c d} \varphi_{c}(z) \varphi_{d}(w)$. The matrices $R$ determine linear representations of the braid groups on $n$ strings, $B_{n}$, where $n=2,3, \ldots$.

Here, we start by formulating elements of the representation theory of chiral algebras, which allow for a purely representation theoretic construction of the holomorphic and antiholomorphic chiral sectors of rational conformal field theories on $S^{2}$. Postulating the existence of the $R$-matrix implies algebraic relations for its matrix elements; in particular, the conformal dimensions (mod. $\mathbb{Z})$ are determined by $R$. In this representation theoretic approach, the existence of a chiral operator-product algebra is almost evident, and the corresponding structure constants are determined by $R$. Upon combining left and right sectors into a local CFT on $S^{2}$, the locality (or, the crossing symmetry) requirement entails an algebraic equation for the structure constants of the operator-product algebra, an equation which involves the structure constants and $R$ and $\bar{R}$, (the braid matrices of antiholomorphic vertices). In this way, the $R$ and $\bar{R}$ matrices determine completely the structure of local, unitary, rational conformal field theories on $S^{2}$. Similar considerations apply to non-unitary theories.

Apart from partially encoding rational conformal theories, the $R$ matrices associated with a chiral algebra can be used to construct invariants of oriented links $[12,13]$. Furthermore, we conjecture that there is a correspondence between chiral algebras and quantum groups $[22,13]$, such that the $R$-matrices of a chiral algebra generate the commutant of tensor product representations of a corresponding quantum group, where the deformation parameter, $q$, of the quantum group is a root of unity.

Ideas similar to the ones presented in this paper have been developed, in ref. [5].

This paper is organized as follows. (Holomorphic) chiral algebras, equipped with an unusual tensor product on representation spaces, are discussed in the next section. The integers $N_{i j k}$, familiar from the theory of rational CFT, receive a representation theoretic interpretation, since they become the multiplicities in the tensor product decomposition. This decomposition into irreducible representations of the chiral algebra gives rise to Clebsch-Gordan coefficients, which in turn are used, in Sect. 3, to construct the chiral vertex operators $\varphi(z)$. We assume that the structure of the chiral algebra is compatible with the existence of $R$-matrices describing the analytic continuation of products of chiral vertices. Examples of known chiral algebras together with their $R$-matrices are given. In Chap. 4, all those algebraic relations for the $R$-matrices are derived which can be obtained by considering the analytic continuation of products of chiral vertex operators, assuming the chiral field theory to be defined on $S^{2}$. In Sect. 5, the existence of chiral fusion (i.e. of the chiral OPE) on the vacuum, plus a compatibility assumption, lead to the existence of chiral fusion in general. The fusion constants 
are calculated in terms of $R$-matrix elements. A combination of braid and fusion operations on chiral vertices then leads to new identities which are obeyed by $R$. In Sect. 6, some of them are employed to compute explicitly the spectral decomposition of the monodromy matrices, $R^{2}$, and this result allows for a rigorous proof of Vafa's [4] lemma, which states that in a rational CFT the conformal dimensions, $h$, are rational numbers. Chapter 7 contains a brief description of how the $R$-matrices can be used to construct link-invariants. The second part of Chap. 7 is devoted to describing the Vertex-SOS transformation of the $R$-matrices and thus to suggest a general connection between the representation theory of chiral algebras and the one of quantum groups. In Chap. 8 we describe how to combine a holomorphic and an antiholomorphic chiral field theory into a rational, local CFT on $S^{2}$. The locality equation, which determines the operator-product expansion coefficients, $C$, in terms of $R$ and $\bar{R}$, is derived, and the symmetries of $C$ are computed. It is shown how, in simple circumstances, one can easily determine $C$, once one knows $R$ and $\bar{R}$. Some concluding remarks are collected in Sect. 9. In particular, we suggest a way of reconstructing conformal blocks from the $R$-matrices of a quantum group by solving a generalized Riemann-Hilbert problem.

While we were working on the problems discussed in this paper we received very interesting preprints by Moore and Seiberg [5], Rehren and Schroer [24] and others where similar problems are addressed. We thank these colleagues for keeping us informed about their work by sending us their preprints. We have also been informed by I.G. Frenkel about his forthcoming book with Lepowsky and Meurman which is likely to contain ideas and results that overlap with ours. Gervais has pointed out to us that braid matrices also appear in his work with Neveu [25].

We hope the presentation of our results in this paper will still be useful.

\section{Chiral Sectors I: Representations of the Chiral Algebra}

We begin by summarizing some elements of the representation theoretic approach to conformal field theory as sketched in Sect. 5 of Ref. [1]. The presentation in this and the following chapters is restricted to the holomorphic chiral sector, the analysis in the case of the antiholomorphic sector being analogous.

In the Euclidean formulation, an abstract local ${ }^{1}$ chiral algebra $\mathscr{A}$ is generated by Möbius covariant, holomorphic (unbounded) operator-valued fields $\psi_{j}(z)$, where $z \in \mathbb{C}, j \in I$. By Möbius covariance we mean that for each $j \in I$ there is a real number $h_{j}$, and for each $w \in \operatorname{PSL}(2, \mathbb{C})$ (which maps $z \mapsto w(z)$ ) there exists an automorphism $\tau_{w}$ on $\mathscr{A}$, such that

$$
\begin{gathered}
\tau_{w}(A \cdot B)=\tau_{w}(A) \cdot \tau_{w}(B), \\
\tau_{w}\left(\psi_{j}(z)\right)=\left(\frac{d w}{d z}\right)^{h_{j}} \psi_{j}(w(z)),
\end{gathered}
$$

for all $A, B \in \stackrel{\mathscr{A}}{,}, j \in I$.

\footnotetext{
${ }^{1}$ Throughout this paper, we restrict our attention to local chiral algebras. In the case of nonlocal algebras (cf. [1]), the analysis would be somewhat more cumbersome, but essentially parallels the one which we follow here
} 
For the sake of clarity and simplicity we will, later on, consider representations of $\mathscr{A}$ on Hilbert spaces only, thus neglecting the possibility of representing the chiral algebra on spaces of indefinite metric. Thus we require that $\mathscr{A}$ is a $*$-algebra, i.e., by assumption, there is an involution $*$ defined on the index set $I$ such that

$$
\left(\psi_{j}(z)\right)^{*}=\left(\frac{1}{z^{*}}\right)^{2 h_{j}} \psi_{j *}\left(\frac{1}{z^{*}}\right)
$$

Furthermore, $\tau_{w}$ is supposed to act as a $*$ automorphism, i.e. for all $A \in \mathscr{A}$,

$$
\left(\tau_{w}(A)\right)^{*}=\tau_{w_{*}}\left(A^{*}\right),
$$

where $w_{*}\left(\frac{1}{z^{*}}\right):=\frac{1}{w(z)^{*}}\left(\right.$ notice that if $w$ is given by $\left(\begin{array}{ll}a & b \\ c & d\end{array}\right) \in \operatorname{PSL}(2, \mathbb{C})$, then $w_{*}$ is represented by $\left.\left(\begin{array}{ll}d^{*} & c^{*} \\ b^{*} & a^{*}\end{array}\right)\right)$. It is easy to see rhat (2.3) and (2.4) imply that $h_{j}=h_{j^{*}}$ The automorphisms $\tau_{w}$ are assumed to be generated by a field $T(z), T^{*}=T$, having conformal dimension $h_{T}=2$, such that the Fourier coefficients $L_{n}$ of $T(z)$ obey the following commutation relations for $n=-1,0,+1$ :

$$
\left[L_{n}, \psi_{j}(z)\right]=\left(z^{n+1} \frac{\partial}{\partial z}+(n+1) h_{j} z^{n}\right) \psi_{j}(z),
$$

where, for $n \in \mathbb{Z}$,

$$
L_{n}:=\frac{1}{2 \pi i} \oint_{|z|=1} d z T(z) z^{n+1} .
$$

We assume that the identity operator $\mathbb{1}=\mathbb{1}^{*}$, which is independent of $z$ and consequently has $h_{\mathbb{1}}=0$, is contained in $\mathscr{A}$.

Requiring that $\mathscr{A}$ is local means that, for arbitrary $\left(z, z^{\prime}\right) \in \mathbb{C}^{2} \backslash D_{2}, D_{2}$ denoting the diagonal $\left\{(z, z) \in \mathbb{C}^{2}\right\}$, and for all $(j, k) \in I \times I$,

$$
\psi_{j}(z) \psi_{k}\left(z^{\prime}\right)=\psi_{k}\left(z^{\prime}\right) \psi_{j}(z)
$$

We wish to point out that the conformal dimensions, $h_{j}$, must be natural numbers, as implied by the Möbius covariance of the fields $\psi_{j},(2.5)$, and their statistics, (2.7), under the condition that some representation theoretic requirements (cf. (2.31)) are obeyed.

Thus, the chiral algebra $\mathscr{A}$ is generated by local currents of $\operatorname{spin} h=0,1,2, \ldots$. Since $\mathscr{A}$ is an algebra of local currents it makes sense to define the Fourier coefficients of $\psi_{j}(z)$ by

$$
\psi_{j, n}:=\frac{1}{2 \pi i} \oint_{|z|=1} d z z^{h_{j}+n-1} \psi_{j}(z),
$$

where $n \in \mathbb{Z}$, and for all $j \in I$. Taking into account that $\mathscr{A}$ is a $*$-algebra, (2.3) yields

$$
\left(\psi_{j, n}\right)^{*}=\psi_{j^{*},-n} .
$$

Definition. The complex algebra with unit, which is generated by $\left\{\psi_{j, n} \mid j \in I, n \in \mathbb{Z}\right\}$, and which is spanned by all (formal) polynomials in the generators, is denoted by $\mathscr{A}$. 
Remark. We note that, in general, there will be polynomial relations on $\mathscr{A}$; such as commutation relations which characterize infinite-dimensional Lie algebras (Virasoro, affine Lie algebras,...), or even non-Lie-algebra type relations (e.g. Zamolodchikov's spin-3 algebra [2]).

We need to discuss the properties of $\mathscr{A}$ in some detail. Because $T^{*}$ equals $T$, we get $L_{n}^{*}=L_{-n}$. From the commutation relations (2.5) we deduce that $L_{0}=L_{0}^{*}$ generates rotations $z \mapsto e^{i \sigma} z, \sigma \in \mathbb{R}$, and scale transformations $z \mapsto \lambda z, \lambda \in \mathbb{R}$. Using (2.8) and (2.5) it is easily verified that

$$
\left[L_{0}, \psi_{j, n}\right]=-n \psi_{j, n} .
$$

Thus, the set of generators $G=\left\{\psi_{j, n} \mid j \in I, n \in \mathbb{Z}\right\}$ is $\mathbb{Z}$-graded, $G=G_{<} \cup G_{0} \cup G_{>}$, where $G_{<}=\bigcup_{n \in \mathbb{N}^{+}} G_{n}, G_{>}=\bigcup_{-n \in \mathbb{N}^{+}} G_{n}$, and by definition $G_{m}=\bigcup_{j}\left\{\psi_{j, m}\right\}$. From its generators the algebra $\mathscr{A}$ inherits the equivalent $\mathbb{Z}$-grading, namely

$$
\mathscr{A}=\bigoplus_{n \in \mathbb{Z}} \mathscr{A}_{n},
$$

where $\mathscr{A}_{n}$ is the $\mathbb{C}$-span of all elements $A \in \mathscr{A}$ which obey

$$
\left[L_{0}, A\right]=-n \cdot A \text {. }
$$

For $n=-1,0,+1,(2.5)$ and (2.8) yield

$$
\left[L_{n}, \psi_{r, s}\right]=\left(-s+n\left(h_{r}-1\right)\right) \psi_{r, s+n},
$$

and thus if, for some $s>\left|h_{r}-1\right|, \psi_{r, s}=0$, then $\psi_{r, s^{\prime}}=0, \forall s^{\prime} \geqq-\left|h_{r}-1\right|$; and similarly for $s<-\left|h_{r}-1\right|$ (assuming $h_{r} \geqq 1$ ).

On the linear hull of $G$ we define a family of linear deformation maps $\left\{\delta_{z} \mid z \in \mathbb{C}^{*}\right\}$ as follows:

$$
\begin{gathered}
\delta_{z}: \operatorname{span}_{\mathbb{C}}(G) \rightarrow \operatorname{span}_{\mathbb{C}}(G) \\
\delta_{z}\left(\psi_{r, s}\right):=\sum_{k=1-h_{r}}^{\infty} z^{s-k}\left(\begin{array}{l}
h_{r}+s-1 \\
h_{r}+k-1
\end{array}\right) \psi_{r, k} .
\end{gathered}
$$

Of course, $\delta_{z}\left(\psi_{r, s}\right):=0$, if $\psi_{r, s}=0$. For example, we have

$$
\begin{aligned}
\delta_{z}(\mathbb{1}) & =0, \\
\delta_{z}\left(L_{-1}\right) & =L_{-1}, \\
\left.\delta_{z}\left(L_{n}\right)\right|_{n \geqq-1} & =z^{n+1} L_{-1}+(n+1) z^{n} L_{0}+\ldots+L_{n}, \\
\left.\lim _{z \rightarrow 0} \delta_{z}\left(\psi_{r, s}\right)\right|_{s \geqq 1-h_{r}} & =\psi_{r, s} .
\end{aligned}
$$

It is important to note that $\delta_{z}$ is not $a *$-endomorphism, since in general $\left(\delta_{z}\left(\psi_{r, s}\right)\right)^{*}$ $\neq \delta_{z}\left(\left(\psi_{r, s}\right)^{*}\right)$.

\section{Lemma 1.}

a)

$$
\begin{gathered}
{\left[L_{-1}, \delta_{z}\left(\psi_{r, s}\right)\right]=-\frac{d}{d z} \delta_{z}\left(\psi_{r, s}\right)} \\
e^{-L_{-1}(z-w)} \delta_{w}(\cdot) e^{+L_{-1}(z-w)}=\delta_{z}(\cdot), \\
\delta_{z} \circ \delta_{w}=\delta_{z+w} .
\end{gathered}
$$$$
\text { c) }
$$ 


\section{Proof.}

a) This follows directly from (2.13) and (2.14).

b) Equation (2.17) is the integrated form of (2.16).

c) An easy computation.

In the statement as well as during the proof of Lemma 1 we have been careless about the allowed range of the points $z$ and $w$. This is easily repaired, since $\delta_{z}$ is defined for $0<|z|<\infty$.

These deformation maps are now employed in order to endow $\mathscr{A}$ with the structure resembling that of a Hopf algebra. We introduce a family of comultiplications $\left\{\Delta_{z}: \mathscr{A} \rightarrow \mathscr{A} \otimes \mathscr{A} \mid z \in \mathbb{C}^{*}\right\}$, defined by

$$
\Delta_{z}\left(\psi_{r, s}\right)=\delta_{z}\left(\psi_{r, s}\right) \otimes 1+1 \otimes \psi_{r, s},
$$

when acting on generators. It is a basic assumption of this approach to conformal field theory that $\Delta_{z}$ can be extended consistently to a morphism of algebras (although not a $*$-morphism). I.e. we assume that

$$
\begin{gathered}
\Delta_{z}\left(\sum_{i=1}^{N} A_{i}\right):=\sum_{i=1}^{N} \Delta_{z}\left(A_{i}\right), \\
\Delta_{z}\left(\prod_{i=1}^{N} \psi_{r_{i}, s_{i}}\right):=\prod_{i=1}^{N} \Delta_{z}\left(\psi_{r_{i}, s_{i}}\right),
\end{gathered}
$$

is a definition which is consistent with the relations obeyed by elements of $\mathscr{A}$. Here, $A_{i}$ is some monomial in the generators. Clearly, $\Delta_{z}(\alpha \cdot A)=\alpha \cdot \Delta_{z}(A)$, for $\alpha \in \mathbb{C}$, which follows from the linearity of $\delta_{z}$ and from (2.20). It is a fact that (2.20) is consistent for all known chiral local algebras of conformal field theory, e.g. Virasoro algebras, affine Lie algebras, ... . It is important to point out that if there is a relation

$$
\sum_{i=1}^{n} \lambda_{i} A_{i}=c \cdot \mathbb{1}, \quad \lambda_{i} \in \mathbb{C}, \quad c \in \mathbb{C},
$$

obeyed in $\mathscr{A}$, then, since $\Delta_{z}(\mathbb{1})=\mathbb{1} \otimes \mathbb{1}$,

$$
\sum_{i=1}^{n} \lambda_{i} \Delta_{z}\left(A_{i}\right)=c \cdot \mathbb{1} \otimes \mathbb{1}
$$

Therefore, the morphisms $\Delta_{z}$ map $\mathscr{A}$ into some subalgebra, $\Delta_{z}(A)$, of $\mathscr{A} \otimes \mathscr{A}$, where the values of the central extensions of $\Delta_{z}(A)$ equal the values of the central extensions in the second factor of $\mathscr{A} \otimes \mathscr{A}$.

In general, we have not checked and thus assume that

$$
\operatorname{ker}\left(\Delta_{z}\right)=0, \quad \forall z \in \mathbb{C}^{*} .
$$

It is easy to see that we get the commutative diagram,

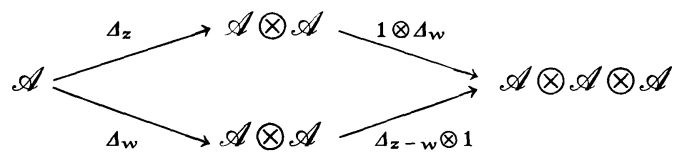

which states that $\left(\mathscr{A},\left\{\Delta_{z}\right\}\right)$ is some modification of a Hopf algebra. 
We remark that $\left(\tilde{\mathscr{A}}, \Delta_{0}\right), \tilde{\mathscr{A}}$ being the algebra generated by $\left\{\psi_{r, s} \mid s \geqq 1-h_{r}\right\}$, is indeed a Hopf algebra, which is a triviality in view of (2.15) and (2.20) (extended to $z=0$ ).

From the point of view of two-dimensional unitary conformal field theory we are interested in irreducible, unitary, positive-energy representations, $\varrho_{k}$, of $\mathscr{A}$ on separable Hilbert spaces $\mathscr{H}_{k}$. More precisely, $\varrho_{k}$ is supposed to obey the following requirements:

(a) $\varrho_{k}$ is unitary, i.e. $\varrho_{k}\left(A^{*}\right)=\left(\varrho_{k}(A)\right)^{*}$, for all $A \in \mathscr{A}$.

(b) $\varrho_{k}$ is covariant, i.e. $\varrho_{k}\left(\tau_{w}(A)\right)=U_{k}(w) \varrho_{k}(A) U_{k}(w)^{-1}$, where the representation, $U_{k}(w)$, of the Möbius transformation is supposed to be defined on some $\varrho_{k}(\mathscr{A})$ invariant domain which is dense in $\mathscr{H}_{k}$, if $w$ is sufficiently close to the identity in $\operatorname{PSL}(2, \mathbb{C})$.

(c) positive-energy: Rotations through an angle $\sigma$ are represented by a unitary operator $U_{k}(\sigma)=\exp \left(i L_{0}^{k} \sigma\right)$, where $L_{0}^{k} \equiv \varrho_{k}\left(L_{0}\right)$ is an (unbounded) selfadjoint, positive-semidefinite operator on $\mathscr{H}_{k}$.

(d) The spectrum of $L_{0}$ on $\mathscr{H}_{k}$, $\operatorname{spec}_{k}\left(L_{0}\right)$, is pure point.

Remark. It would have been sufficient to demand (d) and to replace (c) with a milder version, in which one postulates that $L_{0}^{k}$ be bounded from below [1].

As a rule, we will refrain from worrying much about domain problems. Such problems are likely to occur, since the representation $\varrho_{k}$ will in general be a *-morphism from $\mathscr{A}$ into unbounded operators on $\mathscr{H}_{k}$. We thus assume that all the operators considered, as well as products of them, make sense and have a dense domain of definition. iff

A vector $v \in \mathscr{H}_{k}, v \neq 0$, is said to be $\mathscr{A}$-invariant (or, for simplicity, just invariant),

$$
\varrho_{k}\left(\mathscr{A}_{n}\right) v=0, \quad \forall n>0 .
$$

The postulates (c) and (d) then imply that a vector in $\mathscr{H}_{k}$ is invariant if and only if it is a lowest weight vector for $L_{0}^{k}$. It is easy to verify this statement, having in mind that each nonzero vector in $\mathscr{H}_{k}$ is a cyclic vector (because $\varrho_{k}$ is an irreducible representation on a Hilbert space). We define

$$
H_{k}:=\inf \operatorname{spec}_{k}\left(L_{0}\right) \text {. }
$$

Part (d) and the locality of $\dot{\mathscr{A}}$ tell us that

$$
\operatorname{spec}_{k}\left(L_{0}\right) \subset\left\{H_{k}+\mathbb{N}\right\} \text {. }
$$

Let $L$ be a finite index set, where $k \in L$ means that $\varrho_{k}$ is an irreducible, unitary, positive-energy representation of $\mathscr{A}$ on some separable Hilbert space $\mathscr{H}_{k}$. We assume that $\left\{\mathscr{H}_{k} \mid k \in L\right\}$ is closed under $\otimes$, in the sense that for all $j \in L, k \in L$,

$$
\mathscr{H}_{j} \otimes \underset{z}{ } \mathscr{H}_{k} \sim \underset{i \in L}{\bigoplus_{\alpha=1}^{N_{i j k}}} \mathscr{H}_{i}^{(\alpha)}
$$

This requires some explanation. First of all, the tensor product $\mathscr{H}_{j} \otimes \mathscr{H}_{k}$ is defined to be the standard tensor product of Hilbert spaces, but with a non-standard action of the chiral algebra on it. Namely, the label $z$ indicates that the tensor 
product space is subjected to $\Delta_{z}(\mathscr{A})$; and, correspondingly, the right-hand side of (2.27) stands for the decomposition of $\mathscr{H}_{j} \otimes \mathscr{H}_{k}$ into irreducible components for $\Delta_{z}(\mathscr{A})$. The integers $N_{i j k}=0,1,2, \ldots$ count the finite multiplicity of $\mathscr{H}_{i}$ in $\mathscr{H}_{j} \otimes \mathscr{H}_{k}$; and, a priori, $N_{i j k}$ might depend on $z$. However, we will show that the integers $N_{i j k}$ do not depend on $z$, but it is convenient to postpone the proof a bit. Finally, " " in (2.27) indicates that $\mathscr{H}_{j} \otimes \mathscr{H}_{k}$ may contain subspaces carrying non-decomposable representations which are not included on the right-hand side, [12].

If follows from a remark made earlier (cf. (2.22)) that if (2.27) holds, then, if $\mathscr{Z} \in \mathscr{A}$ stands for any central element $\mathscr{Z}$ in $\mathscr{A}$ and if $\varrho_{m}(\mathscr{Z})=\mathbb{1} \cdot \mathscr{Z}_{m},(2.27)$ implies $\mathscr{Z}_{k}=\mathscr{Z}_{i}^{(\alpha)}, \forall i, \alpha$.

Examples. Important examples for a chiral algebra and index sets $L$, which satisfy (2.27), are: $\mathscr{A}=$ Virasoro algebra, and $L$ labels the unitary representations with central charge $c=1-\frac{6}{m(m+1)}$, i.e. $L \equiv L(m), m=3,4, \ldots \mathscr{A}=s \hat{u}_{k}(2)$, and $L(k)$ contains precisely those unitary representations of $\mathscr{A}$ whose spin, $j$, obeys $0 \leqq 2 j \leqq k$.

In analogy to (2.27), a $n$-fold tensor product $\mathscr{H}_{i_{1}} \otimes \mathscr{H}_{i_{2}} \otimes \ldots \otimes \mathscr{H}_{i_{n}}$ will be considered as a representation space for $\mathscr{A} \subset \mathscr{A} \otimes \ldots \otimes \mathscr{A}$, by embedding $\mathscr{A}$ canonically by

$$
(\underbrace{1 \otimes \ldots \otimes 1}_{n-2} \otimes \Delta_{z_{n-1}})(\underbrace{1 \otimes \ldots \otimes 1}_{n-3} \otimes \Delta_{z_{n-2}}) \cdot \ldots \cdot\left(1 \otimes \Delta_{z_{2}}\right) \Delta_{z_{1}}
$$

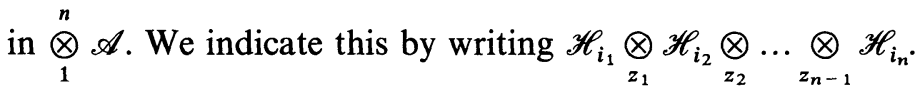

Let $\mathscr{L}$ be an index set $L$ which fulfills one more postulate: There is precisely one index, say 1 , in $\mathscr{L}$, such that $\mathscr{H}_{1}$ contains a Möbius invariant vector; and

$$
\text { this vector is unique (up to normalization, of course). }
$$

We normalize this vector such that it has norm 1 , and call it $\Omega$. Because of the commutation relations of the Möbius generators

$$
\left[L_{n}^{1}, L_{m}^{1}\right]=(n-m) L_{n+m}^{1},
$$

for $n, m=-1,0,+1$, and because $L_{-1}^{1}=\left(L_{1}^{1}\right)^{*}$, we conclude that $L_{0}^{1} \Omega=0$. Since $L_{0}^{1}$ is supposed to be positive semidefinite, and since the eigenspace corresponding to inf $\operatorname{spec}_{1}\left(L_{0}\right) \equiv 0$ is 1 -dimensional, the remarks which follow (2.24) show that $\Omega$ is the unique invariant vector in $\mathscr{H}_{1}$.

Assume that $\Omega$ is separating for $\mathscr{A}$, i.e., for all $z \neq w$,

$$
\left\langle\Omega, \psi_{r *}(w) \psi_{r}(z) \Omega\right\rangle=0 \Leftrightarrow \psi_{r}=0 .
$$

\section{Corollary.}

$$
h_{r} \in \mathbb{N}, \text { for all } r \in I \text {. }
$$

Proof. By the assumption above, the function $\left\langle\Omega, \psi_{r^{*}}(w) \psi_{r}(z) \Omega\right\rangle$ exists, and it is determined fully by scale and translation covariance. It is proportional to 
$(w-z)^{-2 h_{r}}$, and the locality condition (2.7) implies that $h_{r} \in \mathbb{Z}$. Finally, the vector $\psi_{r}(0) \Omega \in \mathscr{H}_{1}$ has conformal weight $h=h_{r}$ (by (2.5)), and the non-negativity of $L_{0}^{1}$ leads to (2.31).

For the purpose of developing a general theory of rational conformal field theories, it is advantageous to impose some further "regularity" conditions on the vacuum $\Omega$ and on the chiral algebra. We summarize the whole structure in the

Definition. A pair $(\mathscr{A}, \mathscr{L})$, consisting of a local, Möbius covariant *-algebra and an index set $\mathscr{L}$ is called rational, if

(a) $\mathscr{A}_{0}$ is generated by $G_{0}$; and $\psi_{\mathrm{r}, 0} \Omega=0$, if $h_{\mathrm{r}} \neq 0$.

(b) $\mathscr{L}$ is a finite index set, such that (2.27), (2.28), and (2.30) hold true.

(c) Adjointness: For each $k \in \mathscr{L} \exists$ ! $j, j \in \mathscr{L}$, such that $N_{1 k j} \neq 0$.

We write $j=k^{*}$, and impose $N_{1 k k^{*}}=1$.

(d) Selection rules: For all $j \in \mathscr{L}, j \neq 1: N_{j j 1} \geqq 1$.

Furthermore, if, for $i \neq 1, N_{i 11} \neq 0$, then $H_{i} \notin \mathbb{N}$.

Remarks.

1. The property (2.30) of $\Omega$ implies, that $\psi_{r}(z)=$ const $\cdot \mathbb{1}$, iff $h_{r}=0$.

Proof. $L_{0}\left(\psi_{r}(0) \Omega\right)=0$ means that we can rescale $\psi_{r}$ such that $\psi_{r}(0) \Omega=\Omega$. The twopoint function $\left\langle\Omega,\left(\psi_{r^{*}}(z)-1\right)\left(\psi_{r}(w)-1\right) \Omega\right\rangle$ is independent of $z$ and $w$, since $h_{r}=0$. But for $w \rightarrow 0$, it simply vanishes, thus it is identically zero, and (2.30) yields the claim.

Therefore, (2.32) takes into account all nontrivial generators $\psi_{r}(z)$.

2. We will show later on that, in fact, the mapping *, which is introduced in (2.34), is an involution on $\mathscr{L}$, and $\mathscr{L} \ni 1=1 *$.

3. The associativity of the tensor product $\mathscr{H}_{j} \otimes \mathscr{H}_{m} \underset{w}{\otimes} \mathscr{H}_{l}$ entails the equation $\sum_{k} N_{i j k} N_{k m l}=\sum_{k^{\prime}} N_{i k^{\prime} l} N_{k^{\prime} j m}$. Towards the end of the next chapter we will require that, among others, $\mathscr{H}_{j} \underset{z}{\otimes} \mathscr{H}_{l} \cong \mathscr{H}_{l} \otimes \mathscr{H}_{j}$, which certainly means that $N_{i j k}=N_{i k j}$. Taken together, we then have $\sum_{k} N_{i j k} N_{k l m}=\sum_{k^{\prime}} N_{i l k^{\prime}} N_{k^{\prime} j m}$, which will have some
important consequences. 4. Notice that if $\mathscr{H}_{i}$ is inequivalent to $\mathscr{H}_{j}$, for $i \neq j$, then the selection rule $N_{j j 1} \geqq 1(j$ $\neq 1$ ) is automatically fulfilled (c.f. also the proof of Lemma 4).

Examples. The most studied and well known prototypes for rational pairs $(\mathscr{A}, \mathscr{L})$ are again: $\mathscr{A}=$ Virasoro algebra, $\mathscr{L}=\mathscr{L}(m) ; \mathscr{A}=s \hat{u}_{k}(2), \mathscr{L}=\mathscr{L}(k)$.

The multiplicities $N_{i j k}$ have become quite popular in the large group of people who work on the classification and analysis of rational conformal field theory (c.f., e.g., [3-5]), although their proper definition remained somewhat murky. Ideas similar to ours have been developed in the last reference of [5]. 


\section{Chiral Sectors II: Construction of Chiral Vertices}

Consider a rational pair $(\mathscr{A}, \mathscr{L})$. The tensor product decomposition (2.27) gives rise to projections $P_{i j k}^{\alpha}(z)$, and their matrix elements, the Clebsch-Gordan coefficients of $\mathscr{A}$, provide a means for the definition of the holomorphic chiral vertex operators.

We define $P_{i j k}^{\alpha}(1)$ to be the unique projection, $\alpha=1, \ldots, N_{i j k}$,

$$
P_{i j k}^{\alpha}(1): \underset{1}{\mathscr{H}_{j} \otimes} \mathscr{H}_{k} \rightarrow \mathscr{H}_{i}^{(\alpha)} \cong \mathscr{H}_{i}
$$

which satisfies

$$
A P_{i j k}^{\alpha}(1)=P_{i j k}^{\alpha}(1) \Delta_{1}(A),
$$

for all $A \in \mathscr{A}$. Here and in the sequel we will often use somewhat sloppy notation in equations such as (3.2), which, in principle, should be written as $\varrho_{i}(A) P_{i j k}^{\alpha}(1)$ $=P_{i j k}^{\alpha}(1)\left(\varrho_{j} \otimes \varrho_{k}\right) \Delta_{1}(A)$.

\section{Proposition 2.}

a)

$$
P_{i j k}^{\alpha}(z)=P_{i j k}^{\alpha}(1)\left(e^{L_{-1}(z-1)} \otimes \mathbb{1}\right),
$$

for $z$ in some neighbourhood, $\mathscr{U}_{\varepsilon}(1)$, of $1 ; \varepsilon<1$.

b)

$$
\frac{d}{d z} N_{i j k}(z)=0, \quad z \in \mathbb{C}^{*}
$$

c) $P_{i j k}^{\alpha}(z)$ is, in general, unbounded.

Proof.

a) Since $\Delta_{z}$ is a morphism, it is enough to verify (3.3) if $\Delta_{z}$ acts on a generator $\psi_{r, s}$. Referring to (2.17), we have, by (3.2),

$$
\begin{aligned}
\varrho_{i}( & \left(\psi_{r, s}\right) P_{i j k}^{\alpha}(1)\left(\varrho_{j} \otimes \varrho_{k}\right)\left(e^{L-1}(z-1) \otimes \mathbb{1}\right) \\
= & P_{i j k}^{\alpha}(1)\left(\varrho_{j} \otimes \varrho_{k}\right)\left(\Delta_{1}\left(\psi_{r, s}\right)\right)\left(e^{L-1(z-1)} \otimes \mathbb{1}\right) \\
= & P_{i j k}^{\alpha}(1)\left(\varrho_{j} \otimes \varrho_{k}\right)\left(\delta_{1}\left(\psi_{r, s}\right) \otimes \mathbb{1}+\mathbb{1} \otimes \psi_{r, s}\right)\left(e^{L-1(z-1)} \otimes \mathbb{1}\right) \\
= & P_{i j k}^{\alpha}(1)\left(\varrho_{j} \otimes \varrho_{k}\right)\left(e^{L_{-1}(z-1)} \otimes \mathbb{1}\right)\left(e^{-L_{-1}(z-1)} \otimes \mathbb{1}\right) \\
& \cdot\left(\delta_{1}\left(\psi_{r, s}\right) \otimes \mathbb{1}+\mathbb{1} \otimes \psi_{r, s}\right)\left(e^{L_{-1}(z-1)} \otimes \mathbb{1}\right) \\
= & P_{i j k}^{\alpha}(1)\left(\varrho_{j} \otimes \varrho_{k}\right) \cdot\left(e^{L_{-1}(z-1)} \otimes 1\right) \Delta_{z}\left(\psi_{r, s}\right) .
\end{aligned}
$$

Thus, $P_{i j k}^{\alpha}(z):=P_{i j k}^{\alpha}(1)\left(e^{L-1}(z-1) \otimes \mathbb{1}\right)$ maps $\mathscr{H}_{j} \otimes \mathscr{H}_{k} \rightarrow \mathscr{H}_{j} \otimes \mathscr{H}_{k} \rightarrow \mathscr{H}_{i}^{(\alpha)}$, because $\mathscr{H}_{j}$ is irreducible; and it is nonzero iff $P_{i j k}^{\alpha}(1)$ is nonzero, and it commutes with the action of $\mathscr{A}$.

b) The relation (3.3) implies that in a neighbourhood of $1 N_{i j k}(z)$ is constant. However, there is nothing special about $z=1$, and a relation analogous to (3.3) holds in the vicinity of every $w \in \mathbb{C}^{*}$. This gives (3.4).

c) For example, since $L_{-1}$ is not bounded, (3.3) shows that in general $P_{i j k}^{\alpha}(z)$ is not bounded either. 
Definition. On some domain dense in $\mathscr{H}_{j} \underset{z}{\otimes} \mathscr{H}_{k}$, assumed to be independent of $z \in \mathscr{U}_{\varepsilon}(1)$, we define the chiral vertex operators, $\varphi_{i j k}^{\alpha}(z, \xi), \quad \xi \in \mathscr{H}_{j}, \quad z \in \mathscr{U}_{\varepsilon}(1)$, $\alpha=1, \ldots, N_{i j k}$,

$$
\varphi_{i j k}^{\alpha}(z, \xi): \mathscr{H}_{k} \rightarrow \mathscr{H}_{i}
$$

to be the linear operators which are given by

$$
\left\langle\xi_{i}, \varphi_{i j k}^{\alpha}(z, \xi) \xi_{k}\right\rangle:=\left\langle\xi_{i}, P_{i j k}^{\alpha}(z) \xi \otimes \xi_{k}\right\rangle,
$$

where $\xi_{i} \in \mathscr{H}_{i}$, and $\xi_{k} \in \mathscr{H}_{k}$.

The results of Sect. 2 lead to

\section{Proposition 3.}

a)

b)

$$
\begin{array}{cc}
\text { a) } & {\left[\psi_{r, s}, \varphi_{i j k}^{\alpha}(z, \xi)\right]=\varphi_{i j k}^{\alpha}\left(z, \delta_{z}\left(\psi_{r, s}\right) \xi\right) .} \\
\text { b) } & {\left[L_{-1}, \varphi_{i j k}^{\alpha}(z, \xi)\right]=\frac{d}{d z} \varphi_{i j k}^{\alpha}(z, \xi) .} \\
\text { c) } & \varrho_{j}\left(L_{0}\right) \xi=h_{\xi} \cdot \xi \Rightarrow\left[L_{0}, \varphi_{i j k}^{\alpha}(z, \xi)\right]=\left(z \frac{d}{d z}+h_{\xi}\right) \varphi_{i j k}^{\alpha}(z, \xi) . \\
\text { d) } & \varrho_{j}\left(L_{n}\right) \xi=\delta_{n, 0} \cdot h_{\xi} \cdot \xi, \text { for } n \geqq 0 \\
& \Rightarrow\left[L_{n}, \varphi_{i j k}^{\alpha}(z, \xi)\right]=\left(z^{n+1} \frac{d}{d z}+z^{n}(n+1) h_{\xi}\right) \varphi_{i j k}^{\alpha}(z, \xi) . \\
\text { e) } & \sum_{\alpha=1}^{N_{i j k}} a_{\alpha} \cdot \varphi_{i j k}^{\alpha}(z, \cdot)=0 \Leftrightarrow a_{\alpha}=0, \forall \alpha .
\end{array}
$$

e)

Proof.

a) By definition, $\psi_{r, s} \varphi_{i j k}^{\alpha}(z, \xi) \xi_{k}=\psi_{r, s} P_{i j k}^{\alpha}(z)\left(\xi \otimes \xi_{k}\right)$, for $z \in \mathscr{U}_{\varepsilon}(1)$, and $\xi \otimes \xi_{k}$ in some dense domain of $\mathscr{H}_{j} \otimes \mathscr{H}_{k}$. Therefore, this equals $P_{i j k}^{\alpha}(z)\left(\delta_{z}\left(\psi_{r, s}\right) \xi \otimes \xi_{k}+\xi \otimes \psi_{r, s} \xi_{k}\right)$, which in turn gives $\varphi_{i j k}^{\alpha}\left(z, \delta_{z}\left(\psi_{r, s}\right) \xi\right) \xi_{k}+\varphi_{i j k}^{\alpha}(z, \xi) \psi_{r, s} \xi_{k}$.

b) Consider: $\quad \frac{d}{d z}\left\langle\xi_{i}, \varphi_{i j k}^{\alpha}(z, \xi) \xi_{k}\right\rangle \equiv \frac{d}{d z}\left\langle\xi_{i}^{(\alpha)}, P_{i j k}^{\alpha}(1) \quad\left(e^{L_{-1}(z-1)} \otimes 1\right) \quad\left(\xi \otimes \xi_{k}\right)\right\rangle$ $=\left\langle\xi_{i}^{(\alpha)}, P_{i j k}^{\alpha}(z)\left(L_{-1} \xi \otimes \xi_{k}\right)\right\rangle$, which, by (3.7), is identical to $\left\langle\xi_{i},\left[L_{-1}, \varphi_{i j k}^{\alpha}(z, \xi)\right] \xi_{k}\right\rangle$.

c) .If $\xi$ is an eigenstate of $L_{0}$ with eigenvalue $h_{\xi}$, then $\delta_{z}\left(L_{0}\right) \xi=z L_{-1} \xi+L_{0} \xi$ $=z L_{-1} \xi+h_{\xi} \cdot \xi$ gives, by application of (3.7) and (3.8) the announced result.

d) In this case, $\xi$ is a so-called primary state, and since $\delta_{z}\left(L_{n}\right) \xi=z^{n+1} L_{-1} \xi$ $+z^{n}(n+1) L_{0} \xi$, (3.10) follows easily. $\varphi_{i j k}^{\alpha}(z, \xi)$ is a primary field.

e) Assume that the contrary was true, i.e. that there is a nontrivial linear combination of $\varphi_{i j k}^{\alpha}$ 's, $N_{i j k} \geqq 1$, such that their sum vanishes. Then there is a nontrivial linear combination, with

$$
0=\sum_{\alpha=1}^{N_{i j k}} a_{\alpha}\left\langle\xi_{i}^{(\alpha)}, P_{i j k}^{\alpha}(z)\left(\xi \otimes \xi_{k}\right)\right\rangle,
$$

for all $\xi_{i}^{(\alpha)}, \xi, \xi_{k}$. This, however, means that $P_{i j k}^{\alpha}(z)=0, \forall \alpha$, contradicting the assumption that $N_{i j k} \neq 0$.

We have thus learned that infinitesimally, i.e. for $z \in \mathscr{U}_{\varepsilon}(1)$, the chiral vertices are scaling fields, i.e. they are translation and, if $\xi$ is chosen appropriately, scale 
covariant. We now define $\varphi_{i j k}^{\alpha}(z, \xi)$, for $z \in \mathbb{C}^{*}$, by analytic continuation. This can be done easily, and in a manner which gives a $z$-independent domain of definition. For $z \in \mathscr{U}_{\varepsilon}(1)$, and $\xi_{i}, \xi$, and $\xi_{k}$ eigenstates of $L_{0}$, we have, by (3.9),

$$
\left\langle\xi_{i}, \varphi_{i j k}^{\alpha}(z, \xi) \xi_{k}\right\rangle=z^{H_{i}-H_{J}-H_{k}} z^{n}\left\langle\xi_{i}, \varphi_{i j k}^{\alpha}(1, \xi) \xi_{k}\right\rangle,
$$

where the integer $n$ depends on $\xi, \xi_{i}, \xi_{k}$. Thus, formula (3.12) permits a (multivalued) extension of $\left\langle\xi_{i}, \varphi_{i j k}^{\alpha}(z, \xi) \xi_{k}\right\rangle$ from $\mathscr{U}_{\varepsilon}(1)$ to $\mathbb{C}^{*}$. And, by linearity, this defines a (multivalued) analytic continuation of $\varphi_{i j k}^{\alpha}(z, \xi)$ to $\mathbb{C}^{*}$, which preserves (3.7) $-(3.11)$.

$(\mathscr{A}, \mathscr{L})$ is a rational pair. This enables us to relate vectors in $\mathscr{H}_{j}$ to chiral vertices, as $z \rightarrow 0$.

\section{Lemma 4.}

a)

$$
\begin{gathered}
N_{j 11}=\delta_{j 1} . \\
N_{i j 1}=\delta_{i j} . \\
\lim _{z \rightarrow 0}\left(\varphi_{j j 1}(z, \xi) \Omega\right)=v_{j j 1} \cdot \xi,
\end{gathered}
$$

where $v_{j j 1}$ is a normalization constant, $v_{j j 1} \neq 0$.

Proof.

a) Clearly, $\Delta_{z}\left(L_{n}\right)(\Omega \otimes \Omega)=0$, for $n=0, \pm 1$. Thus, $\mathscr{H}_{1} \otimes \mathscr{H}_{1}$ contains a Möbius invariant state, and this is compatible with (2.27) and (2.28), iff $N_{111} \geqq 1$.(2.34) gives $N_{111}=1$, meaning that $1=1^{*}$. Next, suppose that $\varphi_{j 11}^{\alpha}(z) \neq 0$. Thus there are $L_{0^{-}}$ eigenstates $v_{j}, v_{j}=$ invariant vector in $\mathscr{H}_{j}$, and $\xi, \xi^{\prime}$ such that $\left\langle v_{j}, \varphi_{j 11}^{\alpha}(z, \xi) \xi^{\prime}\right\rangle \neq 0 . \xi^{\prime}$ can be reached from $\Omega$ by a polynomial in the generators, and since $\varphi_{j 11}^{\alpha}(z, \xi)$ is linear in $\xi$, this nonvanishing matrix element can be written as

$$
\left\langle v_{j}, \varphi_{j 11}^{\alpha}(z, \xi) \xi^{\prime}\right\rangle=\sum_{i} p_{i}(z)\left\langle v_{j}(i), \varphi_{j 11}^{\alpha}(z, \xi(i)) \Omega\right\rangle .
$$

Here, for each $i, p_{i}(z)$ is a polynomial in $z, z^{-1}$, and $v_{j}(i)$ and $\xi(i)$ are invariant vectors in $\mathscr{H}_{j}$ and some eigenvectors of $L_{0}$ in $\mathscr{H}_{1}$, respectively. Since $L_{-1} \Omega=L_{+1} v_{j}(i)=0$, $\left\langle v_{j}(i), \varphi_{j 11}^{\alpha}(z, \xi(i)) \Omega\right\rangle$ is independent of $z$. But this matrix element is proportional to $z^{H_{j}-h_{\xi(i)}}$, therefore it is zero, or $H_{j}=h_{\xi(i)}$. Since at least one of these matrix elements must, by hypothesis, be nonzero, $H_{j}=h_{\xi(i)}$, for some $i$. Thus, $H_{j} \in \mathbb{N}$, and the selection rule (2.35) yields $N_{j 11}=0$, if $j \neq 1$.

b) Let $v_{j}$ be an invariant vector in $\mathscr{H}_{j}$. As $z \rightarrow 0$, we have $\Delta_{z}\left(\psi_{r, s}\right)\left(v_{j} \otimes \Omega\right) \rightarrow \psi_{r, s} v_{j} \otimes \Omega$, if we remember (2.32), for $s \geqq 0$. We conclude that the selection rule $\mathscr{H}_{j} \subset \mathscr{H}_{j} \otimes \underset{z}{\mathscr{H}_{1}}$ is natural.

Now consider the decomposition of $\mathscr{H}_{j} \otimes \mathscr{H}_{1} \otimes \mathscr{H}_{1}$. Assume that $\mathscr{H}_{j} \otimes \mathscr{H}_{1}$ $=\bigoplus_{m=1}^{M} \mathscr{H}_{i(m)}, M>1$, where multiplicities can appear. The associativity of the tensor product means that

$$
\begin{aligned}
& \mathscr{H}_{j} \otimes \underset{z}{ } \mathscr{H}_{1} \otimes_{w} \mathscr{H}_{1}=\mathscr{H}_{j} \underset{z}{\otimes} \mathscr{H}_{1} \\
& =\bigoplus_{m=1}^{M} \mathscr{H}_{i(m)}, \quad \text { but also }=\bigoplus_{m=1}^{M}\left(\mathscr{H}_{i(m)} \underset{w}{\otimes} \mathscr{H}_{1}\right)=\bigoplus_{n=1}^{N} \mathscr{H}_{k(n)} .
\end{aligned}
$$


Therefore $\quad N=M ; \quad$ thus, $\quad$ for $\quad$ each $\quad m, \quad \mathscr{H}_{i(m)} \otimes \mathscr{H}_{1}=\mathscr{H}_{m^{\prime}}$. Since $j \in\{i(m)\}: \mathscr{H}_{j} \otimes \mathscr{H}_{1}=\mathscr{H}_{j^{\prime}}$, but, since $\mathscr{H}_{j} \otimes \mathscr{H}_{1} \supset \mathscr{H}_{j}$, we finally find, for all $j$ and all $z$ :

$$
\mathscr{H}_{j} \otimes \mathscr{H}_{1}=\mathscr{H}_{j} .
$$

c) By (3.14), there is only one field $\varphi_{j j 1}(z, \xi): N_{j j 1}=1$. Let $\left\{\xi_{n}^{a} \mid n \in \mathbb{N}, a=1,2,3, \ldots\right\}$ be a basis of vectors in the eigenspace of $\varrho_{j}\left(L_{0}\right)$ with eigenvalue $H_{j}+n$. Since $\lim _{z \rightarrow 0}\left(\delta_{z}\left(\psi_{r, s}\right)\right)=\psi_{r, s}$, for $s \geqq 1-h_{r}$, and because $h_{r} \geqq 1$ (apart from the identity operator), and $\psi_{r, 0} \Omega=0$, for $h_{r} \geqq 1$, we get

$$
\lim _{z \rightarrow 0}\left\{L_{0}\left(\varphi_{j j 1}\left(z, \xi_{n}^{a}\right) \Omega\right)\right\}=\left(H_{j}+n\right) \cdot \varphi_{j j 1}\left(0, \xi_{n}^{a}\right) \Omega
$$

and if $A \xi_{n}^{a}=\sum_{b}(A)_{b}^{a} \xi_{n}^{b}$, for $A \in \mathscr{A}_{0}$ (which, by (2.32), is generated by $\left\{\psi_{r, 0}\right\}$ ), we have

$$
\lim _{z \rightarrow 0}\left\{A\left(\varphi_{j j 1}\left(z, \xi_{n}^{a}\right) \Omega\right)\right\}=\sum_{b}(A)_{b}^{a} \varphi_{j j 1}\left(0, \xi_{n}^{b}\right) \Omega .
$$

Thus, the vector $\varphi_{j j 1}\left(0, \xi_{n}^{a}\right) \Omega$ transforms under $\mathscr{A}_{0}$ precisely as $\xi_{n}^{a}$, and therefore is proportional to it (because $\mathscr{A}_{0}$ acts irreducibly on $\left.\operatorname{span}_{\mathbb{C}}\left\{\xi_{n}^{a}\right\}\right) . \varphi_{j j 1}(0, \xi)$ is linear in $\xi$, so the constant of proportionality does not depend on $\xi$. Last, if this constant were zero, $\lim _{z \rightarrow 0}\left\langle\xi_{j}, \varphi_{i j 1}(z, \xi) \Omega\right\rangle=0$, for all $\xi_{j}$, $\xi$, which contradicts $N_{j j 1}=1$.

In order to get rid of the normalization constants $v_{j j 1}$, we consider a specific "gauge", i.e. we change the normalization of $\varphi_{j j 1}$, such that

$$
v_{j j 1}=1 \text {. }
$$

Lemma 5. $\varphi_{j 1 i}^{\alpha}(z, \Omega)$ is a primary field. It commutes with the action of $\mathscr{A}$, if $h_{r} \leqq 1$, $\forall \psi_{r} \neq T$.

Proof.

$$
\left[\psi_{r, s}, \varphi_{j 1 i}^{\alpha}(z, \Omega)\right]=0, \forall s, \quad \text { if } \quad h_{r} \leqq 1 .
$$

This follows from (2.32). Also, $\frac{d}{d z} \varphi_{j 1 i}^{\alpha}(z, \Omega)=0$.

We wish to generalize this behaviour of $\varphi_{j 1 i}^{\alpha}(z, \Omega)$ for the cases where there are higher spin currents. We then assume

$$
\delta_{z}\left(\psi_{r, s}\right) \Omega=0, \text { for all } r, s .
$$

We will see later on that $\varphi_{j 1 i}^{\alpha}=\delta_{i j} \cdot \varphi_{j 1}$, i.e. $N_{j 1 i}=\delta_{i j}$. Equation (3.18), or, more generally, the requirement (3.19) entail

$$
\varphi_{j 1 j}(z, \Omega)=v_{j 1 j} \cdot P_{j},
$$

where the constant $v_{j 1 j}$ is readily gauged to 1 , and $P_{j}$ is the orthogonal projection from the chiral Hilbert space

onto $\mathscr{H}_{j}$.

$$
\mathscr{H}_{\mathscr{L}}:=\underset{i \in \mathscr{L}}{\oplus} \mathscr{H}_{i}
$$


We introduce the conformal blocks, i.e. the $n$-point correlation functions of the holomorphic chiral vertices:

$$
B_{i_{0}, \ldots, i_{n}}^{j_{1}, \ldots, j_{n}}(\alpha, \xi, \mathbf{z}):=\left\langle\Omega, \varphi_{i_{0} j_{1} i_{1}}^{\alpha_{1}}\left(z_{1}, \xi_{1}\right) \ldots \varphi_{i_{n-1} j_{n} i_{n}}^{\alpha_{n}}\left(z_{n}, \xi_{n}\right) \Omega\right\rangle
$$

$\mathbf{z}=\left(z_{1}, \ldots, z_{n}\right), \xi=\left(\xi_{1}, \ldots, \xi_{n}\right), \alpha=\left(\alpha_{1}, \ldots, \alpha_{n}\right)$, and clearly $i_{0}=i_{n}=1$. In (3.22), we take $\left|z_{1}\right|>\left|z_{2}\right|>\ldots>\left|z_{n}\right|$, and the vertices $\varphi_{i_{k-1} j_{k} i_{k}}^{\alpha_{k}}\left(z_{k}, \xi_{k}\right)$ are defined on $-\pi<\arg z_{k}<\pi$ by analytic continuation from 1 to $z_{k}$. In order to simplify our arguments, one might assume that

$$
\varphi_{i j k}(1, \xi) \cdot \lambda^{-L_{0}}
$$

is a bounded operator, for $\lambda>1$. Under this condition the holomorphic conformal blocks "exist" as single-valued analytic functions on

$$
K_{n}^{>}:\left\{\mathbf{z} \in \mathbb{C}^{n}|| z_{1}|>\ldots>| z_{n} \mid,-\pi<\arg z_{j}<\pi\right\} .
$$

We used quotation marks since, again, domain problems, which affect $\xi$, are not dealt with. The statement is rigorously true at least when all $\xi_{k}, 1 \leqq k \leqq n$, are finite linear combinations of eigenvectors of $L_{0}$ (supposed to be in the domain of definition of $\varphi_{i_{k-1} j_{k} i_{k}}^{\alpha_{k}}$ ).

Under certain conditions we can prove that the conformal blocks are linearly independent analytic functions. The next lemmas are meant to prepare the grounds for Proposition 9, where this fact is stated with more precision.

Lemma 6. For each vector $\xi_{k} \in \mathscr{H}_{k}$ there is at least one vector $\xi_{j} \in \mathscr{H}_{j}, \xi_{j}=\xi_{j}(i, \alpha)$, which fulfills

$$
P_{i j k}^{\alpha}(1)\left(\xi_{j} \otimes \xi_{k}\right) \neq 0
$$

if $P_{i j k}^{\alpha}(1) \neq 0$.

Proof. Assume that the contrary were true. Then there exist some $\xi_{k} \in \mathscr{H}_{k}$ and $(\alpha, i)$, such that $P_{i j k}^{\alpha}(1) \neq 0$, and $P_{i j k}^{\alpha}(1)\left(\xi_{j}^{\prime} \otimes \xi_{k}\right)=0$, for all $\xi_{j}^{\prime}$. But then, $P_{i j k}^{\alpha}(1)\left(\xi_{j}^{\prime} \otimes \psi_{r, s} \xi_{k}\right)$ $=\psi_{r, s}\left(P_{i j k}^{\alpha}(1)\left(\xi_{j}^{\prime} \otimes \xi_{k}\right)\right)-P_{i j k}^{\alpha}(1)\left(\delta_{1}\left(\psi_{r, s}\right) \xi_{j}^{\prime} \otimes \xi_{k}\right)=0$, thus $P_{i j k}^{\alpha}(1)\left(\xi_{j}^{\prime} \otimes \xi_{k}^{\prime}\right)=0$, for all $\xi_{k}^{\prime}, \xi_{j}^{\prime}$. This contradicts the assumption that $P_{i j k}^{\alpha}(1) \neq 0$.

It will be proved in Sect. 4 that $N_{1 j j^{*}}=1$. Let us accept this for the moment. Then we may drop the index $\alpha$ from $\varphi_{1 j j^{* *}}^{\alpha}$ We have

\section{Lemma 7.}

a) Let $v_{j^{*}}$ be an invariant vector in $\mathscr{H}_{j^{*}}$ Then there is $\xi_{j} \in \mathscr{H}_{j}$, such that $\left\langle\Omega, \varphi_{1 j j^{*}}\left(1, \xi_{j}\right) v_{j^{*}}\right\rangle \neq 0$.

b) Assume, that $\varphi_{1 j j^{*}}(z, \xi)$ and $\varphi_{j^{*} j^{*} 1}(w, \eta)$ are quasi-primary, and $\left\langle\Omega, \varphi_{1 j j^{*}}(z, \xi) \varphi_{j^{*} j^{*} 1}(w, \eta) \Omega\right\rangle \neq 0$. Then $H_{j}=H_{j^{*}}+n, n \in \mathbb{Z}$.

c) If $v_{i} \in \mathscr{H}_{i}$ is invariant and $\alpha$ given, there is $\xi \in \mathscr{H}_{j}$ and $v_{k} \in \mathscr{H}_{k}$, $v_{k}$ invariant, with

$$
\left\langle v_{i}, \varphi_{i j k}^{\alpha}(1, \xi) v_{k}\right\rangle \neq 0 \text {. }
$$

The proof is easy and will not be reported here. Next, we prove the following easy lemma. 
Lemma 8. There is a sequence $\xi$, such that

$$
B_{\mathbf{i}}^{\mathbf{j}}(\alpha, \xi, \mathbf{z}) \neq 0,
$$

if $\mathbf{j}, \mathbf{i}, \boldsymbol{\alpha}$ are given, and $\varphi_{i_{k-1} j_{k} i_{k}}^{\alpha_{k}} \neq 0, \forall k$.

Proof. Suppose the lemma is false. Then $0=\left\langle\Omega, \varphi_{1 j_{1} i_{1}}^{\alpha_{1}}\left(z_{1}, \xi_{1}\right) \ldots \varphi_{i_{n-1} j_{n} 1}^{\alpha_{n}}\left(z_{n}, \xi_{n}\right) A \Omega\right\rangle$, where $A$ is any polynomial in $\psi_{r, s}, s \leqq 0$, and for all $\xi$.

Corollary. For fixed $\mathbf{j}$, there is $\xi$ with

$$
B_{\mathbf{i}}^{\mathbf{j}}(\alpha, \xi, \mathbf{z}) \equiv 0 \Leftrightarrow \varphi_{i_{k-1} j_{k} i_{k}}^{\alpha_{k}}=0, \text { for some } k .
$$

Combining the previous results we conclude the following proposition.

Proposition 9. Assume, that $N_{i j k} \in\{0,1\}$, and that the selection rules (2.35) are augmented by the requirement, that $N_{i j k} \neq 0 \Rightarrow N_{i^{\prime} j k}=0$, if $\left(H_{i}-H_{i^{\prime}}\right) \in \mathbb{Z}$. Then, keeping $\mathbf{j}$ fixed, and if $\xi$ is as in (3.26), all (not a priori vanishing) conformal blocks $B \mathbf{i}(\xi, \mathbf{z})$ are nonzero and linearly independent analytic functions.

Proof. The remaining part of the proof has been established in Ref. [1].

Remark. As (3.12) clearly shows, for general $N_{i j k}$, Proposition 9 is false.

A different kind of linear independence applies to products of chiral vertices. This is what we are going to discuss next.

We take it for granted, that on $K_{n}^{>}$all the products $\varphi_{i_{0} j_{1} i_{1}}^{\alpha_{1}}\left(z_{1}, \cdot\right) \ldots \varphi_{i_{n-1} j_{n} i_{n}}^{\alpha_{n}}\left(z_{n}, \cdot\right)$, $\mathbf{i}, \mathbf{j}$, and $\alpha$ arbitrary, are densely defined multilinear, operator-valued holomorphic functions. In particular, this is assumed to hold for their vacuum expectation values.

Proposition 10. Keeping $\mathbf{j}, i_{0}, i_{n}$ and $n$ fixed, the nonzero products $\left\{\varphi_{i_{0} j_{1} i_{1}}^{\alpha_{1}}\left(z_{1}, \cdot\right) \ldots \varphi_{i_{n-1} j_{n} i_{n}}^{\alpha_{n}}\left(z_{n}, \cdot\right)\right\}$ are linearly independent. Equivalently, for given $\mathbf{k}$,

$$
\sum_{\boldsymbol{\beta}} \sum_{\mathbf{p}} a_{\mathbf{p}, \boldsymbol{\beta}} \cdot B_{\mathbf{p}}^{\mathbf{k}}(\boldsymbol{\beta}, \cdot \mathbf{z}) \equiv 0 \Leftrightarrow\left\{\begin{array}{l}
a_{\mathbf{p}, \boldsymbol{\beta}}=0, \\
B_{\mathbf{p}}^{\mathbf{k}}(\boldsymbol{\beta}, \cdot, \mathbf{z})=0 .
\end{array} \text { or },\right.
$$

Proof. As usual, the proof is formal in the sense that domain problems are neglected. In the case of the two-point block we have, by (3.15), $a_{j^{*}} \cdot\left\langle\Omega, \varphi_{1 j j^{*}}(z, \xi) \xi^{\prime}\right\rangle=0, \forall\left(\xi, \xi^{\prime}\right)$. This implies $a_{j^{*}}=0$. The 3-point block gives $\sum_{\alpha} a_{\alpha} \cdot\left\langle\Omega, \varphi_{1 j j^{*}}(z, \xi) \varphi_{j^{*} k l}^{\alpha}\left(w, \xi^{\prime}\right) \xi^{\prime \prime}\right\rangle=0, \quad \forall\left(\xi, \xi^{\prime}, \xi^{\prime \prime}\right)$. Since $\varphi_{j^{*} k l}^{\alpha}\left(w, \xi^{\prime}\right) \xi^{\prime \prime} \hat{=} P_{j^{*} k l}^{\alpha}(w)$ $\left(\xi^{\prime} \otimes \xi^{\prime \prime}\right)$, we can always prepare a linear combination $\sum_{\mu} b_{\mu}^{\beta} \cdot \xi_{\mu}^{\prime} \otimes \xi_{\mu}^{\prime \prime} \equiv v^{\beta}$, defined by $P_{j^{*} k l}^{\alpha}\left(v^{\beta}\right)=\delta_{\alpha, \beta} \cdot P_{j^{*} k l}^{\beta}\left(v^{\beta}\right)$. Because $\mathscr{H}_{j^{*}} \cong$ closure $\left\{P_{j^{*} k l}^{\beta}\left(v^{\mu}\right) \mid\right.$ any $\left.v^{\beta}\right\}$, and since

$$
\begin{aligned}
0 & =\sum_{\mu} b_{\mu}^{\beta} \sum_{\alpha} a_{\alpha}\left\langle\Omega, \varphi_{1 j j^{*}}(z, \xi) \varphi_{j^{*} k l}^{\alpha}\left(w, \xi_{\mu}^{\prime}\right) \xi_{\mu}^{\prime \prime}\right\rangle \\
& =a_{\beta} \cdot\left\langle\Omega, \varphi_{1 j j^{*}}(z, \xi) P_{j^{*} k l}^{\beta}\left(v^{\beta}\right)\right\rangle, \forall\left(\xi, v^{\beta}\right),
\end{aligned}
$$

we get (3.27).

For $(n \geqq 4)$-point blocks, the proof is analogous.

In principle, scale and translation covariance permit us to continue analytically the $n$-point blocks $B_{\mathbf{i}}^{\mathbf{j}}(\boldsymbol{\alpha}, \xi, \mathbf{z})$ from $K_{n}^{>}$to $M_{n}, M_{n} \equiv\left\{\mathbf{z} \in \mathbb{C}^{n} \mid \mathbf{z} \equiv\left(z_{1}, \ldots, z_{n}\right)\right.$ and $z_{i}$ 
$\neq z_{j}$, for $\left.i \neq j\right\}$. The resulting functions are single-valued on the universal covering, $\tilde{M}_{n}$, of $M_{n}$, but in general multi-valued on $M_{n}$ [1]. In order to describe more precisely this analytic continuation we address the 4-point blocks. They are translation-invariant and, for suitable arguments $\xi$, also scale-covariant multilinear functionals on $\mathscr{H}_{j_{1}} \otimes \ldots \otimes \mathscr{H}_{j_{4}}$. Since they are linearly independent (by (3.27)), we might impose the constraint that they form a basis in the linear space of all quadrilinear, translation and scale covariant functionals on $\mathscr{H}_{j_{1}} \otimes \ldots \otimes \mathscr{H}_{j_{4}}$. This constraint is rather powerful, and it allows for a proof of formula (3.28) below. However, this completeness assumption is considerably stronger than assuming directly the validity of Eq. (3.28). Because (3.28) is what we really aim at we assume:

As an operator equation on some domain dense in $\mathscr{H}_{j} \otimes \mathscr{H}_{l} \otimes \mathscr{H}_{m}$, and for $z \neq w$, the analytic continuation along the paths $\gamma_{ \pm}, \mathscr{A}_{ \pm}$, of the product $\varphi_{i j k}^{\alpha}(z, \cdot) \varphi_{k l m}^{\beta}(w, \cdot)$ is given by

$$
\mathscr{A}_{ \pm}\left(\varphi_{i j k}^{\alpha}\left(z, \xi_{j}\right) \varphi_{k l m}^{\beta}\left(w, \xi_{l}\right)\right)=\sum_{\gamma, \delta, k^{\prime}} R^{ \pm}(i j l m)_{k \alpha \beta}^{k^{\prime} \gamma \delta}\left(\varphi_{i l k^{\prime}}^{\gamma}\left(z, \xi_{l}\right) \varphi_{k^{\prime} j m}^{\delta}\left(w, \xi_{j}\right)\right),
$$

for all $\xi_{j}$, $\xi_{l}$. The paths $\gamma_{ \pm}$are depicted in Fig. 1 .

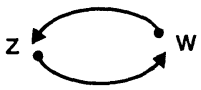

Fig. 1

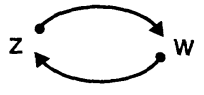

$\gamma-$

The braid matrices $R^{+}(i j l m)$ and $R^{-}(i j l m)$ are $\mathbb{C}$-number matrices, independent of $\xi_{j}$ and $\xi_{l}$.

It is evident from Proposition 10, that the matrices $R^{ \pm}(i j l m)$ may be considered as linear mappings from a linear space $W_{i l j m}$ into $W_{i j l m}$, which have, respectively, the dimensions $\sum_{k^{\prime}} N_{i l k^{\prime}} \cdot N_{k^{\prime} j m}$ and $\sum_{k} N_{i j k} \cdot N_{k l m}$.

We finish this chapter with a definition.

Definition. A triple $(\mathscr{A}, \mathscr{L}, \mathscr{R})$ is called rational, if

a) $(\mathscr{A}, \mathscr{L})$ is rational.

b) $\Omega$ obeys (3.19).

c) The $R^{ \pm}(i j l m)$ matrices, indicated by $R$, satisfy (3.28).

Examples. The only fully worked out example of a rational triple is the unitary discrete series: $\mathscr{A}=$ Virasoro algebra, $\mathscr{L}=\mathscr{L}(m)$. The explicit calculation of the $R^{ \pm}$-matrices was performed by a Coulomb gas representation of the minimal models [6]. Another example is the $s \hat{u}_{k}(2)$ current algebra [7-9]. In Ref. [9], the existence of the $R$-matrices has been proved, and the spin-1/2 $R$-matrices $R(\cdot 1 / 21 / 2 \cdot)$ have been computed.

\section{Chiral Sectors III: Braid Group on $S^{2}$}

The aim of the present section is to derive some of the consequences of Eq. (3.28), i.e. of the existence of the braid matrices, which stem exclusively from considering the analytic continuation of $n$-point blocks. In particular, the multiplicities $N_{i j k}$ 
will be shown to form a set of commuting matrices, and a relation between the conformal dimensions $H_{i}$ and the braid matrices will emerge.

Upon requiring that the $n$-point blocks are defined over $S^{2}$, we will see that the braid matrices obey a very peculiar relation, which, at this stage of the development, might seem to be independent, and not already implied by (3.28).

To begin with, we prove

\section{Lemma 11.}

$$
\begin{aligned}
& R^{+}(i j l m) R^{-}(\text {iljm })=\left.1\right|_{W_{i j l m}}, \\
& R^{-}(i j l m) R^{+}\left({ }_{i j m}\right)=\left.1\right|_{W_{i j l m}} .
\end{aligned}
$$

Proof. The general method to prove relations such as (4.1) consists of performing the analytic continuation of $n$-point blocks (or monomials in vertex operators) from $\left(z_{1}, \ldots, z_{n}\right) \equiv \mathbf{z}$ to $\left(z_{\sigma(1)}, \ldots, z_{\sigma(n)}\right) \equiv \mathbf{z}^{\sigma}$, where $\sigma$ is an element of the symmetric group on $n$ letters, $S_{n}$. The analytic continuation is performed along two different, but isotopic paths in $M_{n}$. There is an isomorphism between such paths in $M_{n}$ on one hand, and braids with $n$ strings on $\mathbb{E}^{2}[10]$ on the other hand. Namely, two paths in $M_{n}$ are isotopic if and only if the same is true for the corresponding braids. Usually, we will talk about paths, but will picture them as "coloured" braids.

The linear independence of the products $\varphi_{i j k}^{\alpha}(z, \cdot) \varphi_{k l m}^{\beta}(w, \cdot), z \neq w$, (cf. 3.27), and the fact that the paths $\gamma_{1}$ and $\gamma_{2}$ in Fig. 2 are isotopic to the trivial path yield

$$
\begin{aligned}
\mathscr{A}_{\gamma_{1}}\left(\varphi_{i j k}^{\alpha}(z, \cdot) \varphi_{k l m}^{\beta}(w, \cdot)\right) & =\left(R^{+}(i j l m) R^{-}(i l j m)\right)_{k \alpha \beta}^{k^{\prime \prime} \gamma \delta} \cdot \varphi_{i j k^{\prime \prime}}^{\gamma}(z, \cdot) \varphi_{k^{\prime \prime} l m}^{\delta}(w, \cdot) \\
& \stackrel{!}{=} \varphi_{i j k}^{\alpha}(z, \cdot) \varphi_{k l m}^{\beta}(w, \cdot),
\end{aligned}
$$
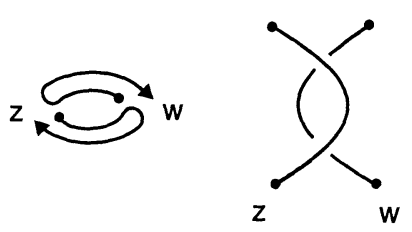

path

braid
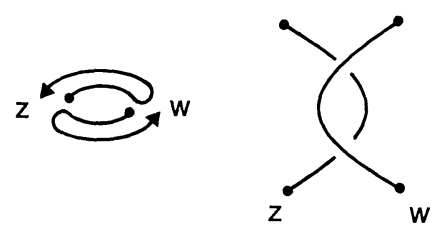

braid

path

Fig. 2

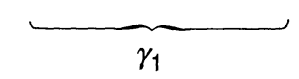

which is fulfilled iff (4.1) is obeyed.

Similarly, $\mathscr{A}_{\gamma_{2}}$ leads to the remaining half of Lemma 11.

Next, since $R^{+}(i j l m) R^{-}($iljm $)=1$ and $R^{+}($iljm $) R^{-}(i j l m)=1$, we get

$$
\operatorname{dim}\left(W_{i j l m}\right)=\operatorname{dim}\left(W_{i l j m}\right) ;
$$

and (4.2) follows from (4.1), and vice-versa.

We now introduce a graphical notation of equations, such as (4.1) and (4.2), in terms of "coloured" braids. To this end we identify $\varphi_{i j k}^{\#}$ with the string of Fig. 3, and in this language there is a $1-1$ correspondence between (4.1), (4.2) and the Reidemeister moves of Fig. 4,

Fig. 3

$$
\left.\varphi_{i j k}^{\#} \sim i\right|_{j} k
$$


We define the matrices $N_{j}$ by

$$
\left(N_{j}\right)_{i k}:=N_{i j k}
$$
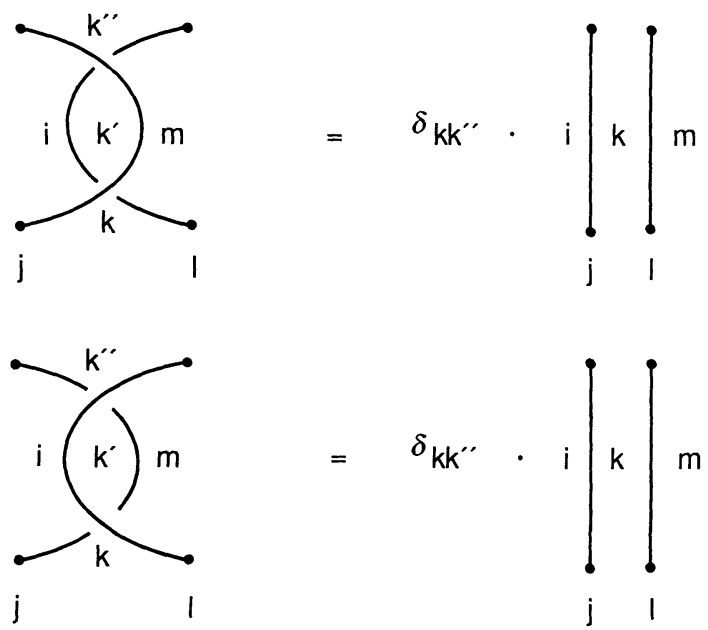

Fig. 4

and their "transposed" $\tilde{N}_{j}$ are given by

$$
\left(\tilde{N}_{j}\right)_{i k}=\left(N_{j}\right)_{k^{*} i^{*}}
$$

\section{Proposition 12.}

a)

b)

c)

d)

$$
\begin{gathered}
N_{i j 1}=\delta_{i j} . \\
{\left[N_{j}, N_{l}\right]=0 .} \\
N_{i j k}=N_{i k j}, \quad N_{i 1 j}=\delta_{i j} . \\
N_{j}=\tilde{N}_{j} .
\end{gathered}
$$

Proof.

a) We just recalled Eq. (3.14), for completeness.

b) This is the master relation. It follows from (4.3):

$$
N_{j} N_{l}=\sum_{k} N_{i j k} N_{k l m}=\operatorname{dim}\left(W_{i j l m}\right)=\operatorname{dim}\left(W_{i l j m}\right)=\sum_{k^{\prime}} N_{i l k^{\prime}} N_{k^{\prime} j m}=N_{l} N_{j} .
$$

c) Consider (4.10), and put $m=1$. Using (4.6) we get $N_{i j k}=N_{i k j}$; put $k=1$, and we have (4.8). In particular, we see that since $\left.N_{1 j j^{*}}=N_{1 j^{*} j}, j^{*}\right)^{*}=j$, and thus $*: \mathscr{L} \rightarrow \mathscr{L}$ is an involution.

d) Consider (4.10), and put $i=1$. We get $N_{1 j j^{*}} N_{j^{*} l m}=N_{1 l^{*}} N_{l^{*} j m}$. By assumption (2.34), $N_{1 j j^{*}}=N_{1 l l^{*}}=1$, for all $j$ and $l$, thus

$$
N_{j^{* l m}}=N_{l^{*} j m} .
$$

We have now $N_{i j k}=N_{j^{*} *^{*} k}$, since $*^{2}=1$, and therefore $N_{i j k}=N_{k^{*} j i^{*}}$

With regard to the matrices $N_{j}$, the simplest case is the one in which all $N_{j}$ are matrices over the set $\{0,1\}$. Recently, matrices over the natural numbers have been 
classified in [11]. There is an A-D-E classification of all matrices over $\{0,1\}$ with euclidean operator norm smaller than 2. Indeed, these norms must equal $2 \cdot \cos \left(\frac{\pi}{q}\right), q=2,3, \ldots$

Example. Assume that $j^{*}=j$, for all $j \in \mathscr{L}$. Also, $|\mathscr{L}|=n$, i.e. $1,2, \ldots, n \in \mathscr{L}$. We know from (4.8) that $N_{1}=\operatorname{diag}(1, \ldots, 1)$ is the $n \times n$ unit matrix. We suppose that the representation $\mathscr{H}_{2}$ obeys $s u(2)$ spin $\frac{1}{2}$ selection rules, i.e.

$$
N_{2}=\left(\begin{array}{cccccc}
0 & 1 & 0 & \ldots & & \\
1 & 0 & 1 & 0 & \ldots & \\
0 & 1 & 0 & 1 & 0 & \ldots \\
\vdots & 0 & 1 & 0 & 1 & \vdots \\
& \vdots & & \ddots & & 1 \\
& & \ldots & 0 & 1 & 0
\end{array}\right) .
$$

Thus, $\left\|N_{2}\right\|=2 \cos \left(\frac{\pi}{n+1}\right)$, and its eigenvalues are $\lambda_{j}=2 \cos \left(\frac{j \pi}{n+1}\right)$, $j=1, \ldots, n$. The components of the eigenvector $v_{j}$ are $v_{j k}=\sin \left(k \cdot \frac{j \pi}{n+1}\right)$. The matrices $N_{1}, \ldots, N_{n}$ are symmetric and commute, thus can be diagonalized simultaneously. Therefore, all

$$
N_{l} \sim\left(\begin{array}{ccc}
\lambda_{1}^{(l)} & & \\
& \cdots & \\
& & \lambda_{n}^{(l)}
\end{array}\right),
$$

and since we know that $\left(N_{l}\right)_{1 j}=\delta_{l j}$, and because also the orthogonal transformation which makes all $N_{l}$ simultaneously diagonal is known, we can calculate the eigenvalues $\lambda_{j}^{(l)}$, and thus get $N_{l}, \forall l$. We obtain

$$
\lambda_{k}^{(l)}=\frac{\sin \left(\frac{k l \pi}{n+1}\right)}{\sin \left(\frac{k \pi}{n+1}\right)}, \quad l=1, \ldots, n,
$$

From this result the selection rules of one half of the chiral sector in the minimal models, or those of $s \hat{u}_{n-1}(2)$, follows:

$$
\mathscr{H}_{i} \otimes \mathscr{H}_{j}=\bigoplus_{k=|i-j|+1}^{\min \{i+j-1,2 n+1-i-j\}} \mathscr{H}_{k} .
$$

If $N_{i j k} \in\{0,1\}$, then some braid matrices can easily be calculated by hand. Suppose, that $\varphi_{i j k}(z, \cdot) \neq 0$. Then $\left\langle\xi_{i}, \varphi_{i j k}\left(z, \xi_{j}\right) \varphi_{k k 1}\left(0, \xi_{k}\right) \Omega\right\rangle \neq 0$, for some vectors $\xi_{i} \in \mathscr{H}_{i}, \xi_{j} \in \mathscr{H}_{j}$, and $\xi_{k} \in \mathscr{H}_{k}$, which are eigenstates of $L_{0}$. This matrix element equals (cf. (3.12)) $z^{H_{i}-H_{j}-H_{k}} \cdot z^{n} \cdot\left\langle\xi_{i}, \varphi_{i j k}\left(1, \xi_{j}\right) \xi_{k}\right\rangle$, and thus, analytically continuing in anti-clockwise orientation $z \rightarrow 0$ and $0 \rightarrow z$ along $\gamma$, we get

$$
\begin{aligned}
\mathscr{A}_{\gamma}\left(\left\langle\xi_{i}, \varphi_{i j k}\left(z, \xi_{j}\right) \xi_{k}\right\rangle\right) & =R^{+}(i j k 1)_{k}^{j} \cdot\left\langle\xi_{i}, \varphi_{i k j}\left(z, \xi_{k}\right) \xi_{j}\right\rangle \\
& =R^{+}(i j k 1)_{k}^{j} \cdot z^{H_{i}-H_{J}-H_{k}} z^{n} \cdot\left\langle\xi_{i}, \varphi_{i k j}\left(1, \xi_{k}\right) \xi_{j}\right\rangle \\
& \stackrel{!}{\equiv}\left(z e^{i \pi}\right)^{H_{i}-H_{J}-H_{k}+n}\left\langle\xi_{i}, \varphi_{i j k}\left(1, \xi_{j}\right) \xi_{k}\right\rangle .
\end{aligned}
$$


We can always rescale the chiral vertex $\varphi_{i j k}$, or $\varphi_{i k j}$, such that for the above triple of vectors $\xi_{i}, \xi_{j}, \xi_{k}$

$$
\left\langle\xi_{i}, \varphi_{i k j}\left(1, \xi_{k}\right) \xi_{j}\right\rangle=(-1)^{n}\left\langle\xi_{i}, \varphi_{i j k}\left(1, \xi_{j}\right) \xi_{k}\right\rangle .
$$

In this gauge, we have

\section{Lemma 13.}

a)

b)

$$
\begin{gathered}
R^{ \pm}(i j l 1)_{l}^{j}=e^{ \pm i \pi\left(H_{i}-H_{j}-H_{l}\right)} . \\
R^{ \pm}(i j 1 m)_{m}^{i}=1, \text { if } \quad N_{i j m} \neq 0 .
\end{gathered}
$$

Proof.

a) We notice that the existence of a matrix $R(i j l m)$ implies that (4.14) must remain true for any other triple of vectors which are eigenstates of $L_{0}$ and for which the matrix element is nonzero.

b) (4.16) is obviously correct for any $N_{i j k}$, since

$$
\begin{aligned}
\varphi_{m 1 m}(z, \Omega) & \equiv \mathbb{1} \quad \text { (cf. (3.20)), i.e. } \\
R^{ \pm}(i j 1 m)_{m \alpha}^{i \beta} & =\delta_{\alpha, \beta} . \quad \square
\end{aligned}
$$

The proof of Lemma 13 parallels the one of

\section{Lemma 13'.}

a)

b)

$$
\begin{gathered}
\sum_{\beta} R^{ \pm}(i j l 1)_{l \alpha}^{j \beta} R^{ \pm}(i l j 1)_{j \beta}^{l \alpha^{\prime}}=\delta_{\alpha \alpha^{\prime}} \cdot e^{ \pm 2 \pi i\left(H_{i}-H_{j}-H_{l}\right)}, \\
R^{ \pm}(i j 1 m)_{m \alpha}^{i \beta}=\delta_{\alpha, \beta} .
\end{gathered}
$$

We notice that $\left(4.15^{\prime}\right)$ does not depend on a specific normalization of the vertices.

The conformal blocks transform, for $\xi$ which are quasiprimary vectors, covariantly under the action of the Möbius group, which is the automorphism group of $S^{2}$. It is thus natural that we wish to interpret the theory under consideration as a theory, which is defined over the 2-sphere. In other words, $S^{2}$ is identified with $\mathbb{C} \cup\left\{\infty\right.$ \} by some conformal mapping $f: S^{2} \rightarrow \mathbb{C} \cup\{\infty\}$, and we think of a $n$-point block $B_{\mathbf{i}}^{\mathbf{i}}(\boldsymbol{\alpha}, \xi, \mathbf{z}), \mathbf{z} \equiv\left(z_{1}, \ldots, z_{n}\right)$, as a function on $S^{2}$, symbolized by $(n+1)$ punctures $f^{-1}\left(z_{1}\right), \ldots, f^{-1}\left(z_{n}\right), f^{-1}(\infty) . f^{-1}(\infty)$ must be included because although the $n$-point block can be continued analytically to $M_{n}$, the point at infinity is, in general, not a point of holomorphy for the conformal blocks. The analytic continuation of the conformal blocks, which is determined by $R^{ \pm}(i j l m)$, must be consistent with the geometry of the surface on which the analytic continuation is performed. It will become handy to define the phases

$$
D_{i j k}^{ \pm}:=e^{\mp 2 \pi i\left(H_{i}-H_{j}-H_{k}\right)} .
$$

Proposition 14. The braid matrices satisfy

$$
R^{+}(i j l m) R^{-}(\text {iljm })=\left.\mathbb{1}\right|_{W_{i j l m}} .
$$

b) The Yang-Baxter-equation (YBE):

$$
\begin{aligned}
& \sum_{k^{\prime}, \alpha^{\prime}, \beta^{\prime}, \beta^{\prime \prime}} R^{+}(i j l m)_{k \alpha \beta}^{k^{\prime} \alpha^{\prime} \beta^{\prime}} R^{+}\left(k^{\prime} j n p\right)_{m \beta^{\prime} \gamma}^{m^{\prime} \beta^{\prime \prime} \gamma^{\prime \prime}} R^{+}\left(i l n m^{\prime}\right)_{k^{\prime} \alpha^{\prime} \alpha^{\prime \prime} \beta^{\prime \prime}}^{k^{\prime \prime} \beta^{\prime \prime \prime}} \\
& =\sum_{k^{\prime}, \gamma^{\prime}, \beta^{\prime}, \beta^{\prime \prime}} R^{+}(k \ln p)_{m \beta \gamma}^{k^{\prime} \beta^{\prime} \gamma^{\prime}} R^{+}\left(i j n k^{\prime}\right)_{k \alpha \beta^{\prime}}^{k^{\prime \prime} \alpha^{\prime \prime} \beta^{\prime \prime}} R^{+}\left(k^{\prime \prime} j l p\right)_{k^{\prime} \beta^{\prime \prime} \gamma^{\prime}}^{m^{\prime} \gamma^{\prime \prime \prime} \gamma^{\prime \prime}} .
\end{aligned}
$$


c) The theory is defined over $S^{2}$, iff

$$
\sum_{k^{\prime} \alpha^{\prime} \beta^{\prime}} R^{+}(i j l m)_{k \alpha \beta}^{k^{\prime} \alpha^{\prime} \beta^{\prime}} D_{k^{\prime} j m}^{-} R^{+}(i l j m)_{k^{\prime} \alpha^{\prime} \beta^{\prime}}^{k^{\prime \prime} \beta^{\prime \prime}}=\delta_{k k^{\prime \prime}} \delta_{\alpha \alpha^{\prime \prime}} \delta_{\beta \beta^{\prime \prime}} \cdot D_{i j k}^{-}
$$

Proof.

a) Equation (4.19) is Lemma 11.

b) The YBE expresses the associativity of analytic continuation: the paths $\gamma_{1}$ and $\gamma_{2}$ in Fig. 5 are isotopic. The linear independence of (the nonvanishing) products $\varphi_{i j k}^{\alpha}(z, \cdot) \varphi_{k l m}^{\beta}(w, \cdot) \varphi_{m n p}^{\gamma}(u, \cdot)$, or, rather, of $\varphi_{i n k^{\prime \prime}}^{\alpha^{\prime \prime}}(z, \cdot) \varphi_{k^{\prime \prime} l m^{\prime}}^{\beta^{\prime \prime \prime}}(w, \cdot) \varphi_{m^{\prime} j p}^{\gamma^{\prime \prime}}(u, \cdot)$, finally gives (4.20).
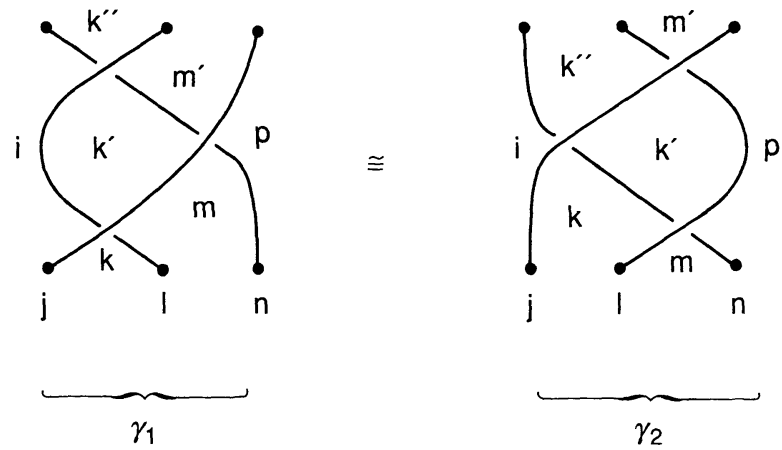

Fig. 5

$\gamma_{1}$

$\gamma_{2}$

c) When we consider $n$-point blocks on the sphere, we have to impose the consistency condition that the paths $\gamma$ and $\gamma^{\prime}$ in Fig. 6 (path picture) and Fig. 7 (braid picture) are isotopic, where $x_{j}=f^{-1}\left(z_{j}\right)$ and $x_{\infty}=f^{-1}(\infty)$.

Fig. 6

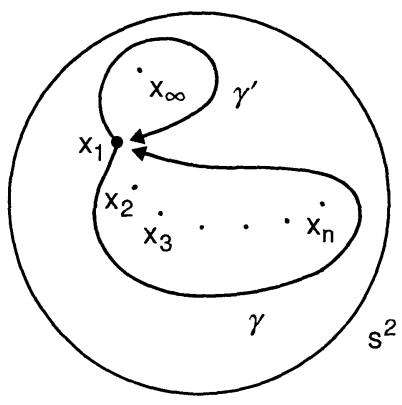

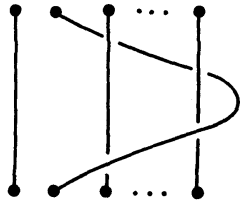

$\infty z_{1} \quad z_{2} \quad z_{n}$

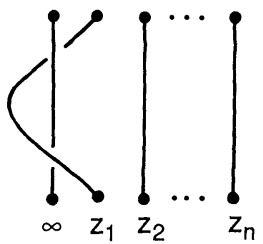

Fig. 7

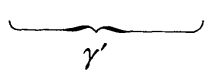


Without loss of generality we may evaluate the analytic continuation of $n$-point blocks whose arguments $\xi$ are eigenstates of $L_{0}$ (since these form a basis in $\left.\mathscr{H}_{j_{1}} \otimes \ldots \otimes \mathscr{H}_{j_{n}}\right)$, and we are allowed to assume that e.g. $\left|z_{1}\right|>\left|z_{2}\right|>\ldots>\left|z_{n}\right|$. The analytic continuation of the $n$-point blocks in $z_{1}$, along $\gamma^{\prime}$, is performed by letting $z_{1} \rightarrow \infty$, replacing $z_{1}=\frac{1}{w_{1}}, w_{1} \rightarrow w_{1} e^{-2 \pi i}$, and going back to the original position of $z_{1}$.

Starting with $n=2$, we obtain a special case of (4.21):

$$
R^{+}\left(1 j^{*} j 1\right)_{j}^{j^{*}} R^{+}\left(1 j j^{*} 1\right)_{j^{*}}^{j}=D_{1 j j^{*}}^{-},
$$

which follows also from $\left(4.15^{\prime}\right)$.

Next, the 3-point block yields

$$
\sum_{\gamma} R^{+}\left(j^{*} i k 1\right)_{k \beta}^{i \gamma} R^{+}\left(j^{*} k i 1\right)_{i \gamma}^{k \delta}=D_{1 i i^{*}}^{-} \cdot \sum_{\alpha} R^{-}(1 j i k)_{j^{*} \beta}^{i^{*} \alpha} R^{-}(1 i j k)_{i^{*} \alpha}^{j^{*} \delta}
$$

With the help of $\left(4.15^{\prime}\right)$ we find

$$
\sum_{\alpha} R^{-}(1 j i k)_{j^{*} \beta}^{i^{*} \alpha} R^{-}(1 i j k)_{i^{*} \alpha}^{*^{*} \alpha}=\delta_{\beta \beta^{\prime}} \cdot D_{k j^{*} i^{*}}^{+},
$$

again a special case of Eq. (4.21).

Let us pause for a moment. If $N_{i j k} \in\{0,1\}$, then we can partially fix the normalization of the vertices by demanding (4.14). However, (4.14) does not fix all the normalizations completely; and Eq. (4.24) suggests - and in fact we could have verified it directly along with the computation of (4.15) - that (4.14) leaves us the freedom to gauge the chiral vertices, such that (4.15) is true and also

$$
R^{ \pm}(1 j l m)_{j^{*}}^{l^{*}}=e^{ \pm i \pi\left(H_{m}-H_{\left.j^{*}-H_{l} *\right)}\right.} .
$$

Finally, the consistency equation which derives from the 4-point block can be brought to a simple form, upon using (4.23). Namely, we get

$$
\begin{aligned}
& \sum_{k^{\prime} \alpha^{\prime} \beta^{\prime} \beta^{\prime \prime}} R^{+}\left(j^{*} i l m\right)_{k \alpha \beta}^{k^{\prime} \alpha^{\prime} \beta^{\prime}}\left(\sum_{\gamma} R^{+}\left(k^{\prime} i m 1\right)_{m \beta^{\prime}}^{i \gamma} R^{+}\left(k^{\prime} m i 1\right)_{i \gamma}^{m \beta^{\prime \prime}}\right) R^{+}\left(j^{*} \lim \right)_{k^{\prime} \alpha^{\prime} \beta^{\prime \prime}}^{k^{\prime \prime} \beta^{\prime \prime}} \\
& \quad=\delta_{k k^{\prime \prime}} \delta_{\beta \beta^{\prime \prime \prime}}\left(\sum_{\gamma} R^{+}\left(j^{*} i k 1\right)_{k \alpha}^{i \gamma} R^{+}\left(j^{*} k i 1\right)_{i \gamma}^{k \alpha^{\prime \prime}}\right) .
\end{aligned}
$$

Combining (4.26) with $\left(4.15^{\prime}\right)$ gives precisely (4.21).

Simple algebra shows that (4.26) is responsible for the fact that there is no new relation stemming from the $n$-point blocks, for $n \geqq 5$.

Define generators $\tau_{0}^{2}, \tau_{1}, \ldots, \tau_{n-1}$, which act on the $n$-point blocks as follows. For $i=1, \ldots, n-1$,

$$
\tau_{i}(B:(\cdot, \cdot, \mathbf{z})):=\mathscr{A}_{\gamma_{i, i+1}}(B:(\cdot, \cdot, \mathbf{z})),
$$

where $\mathscr{A}_{\gamma_{1, i+1}}$ stands for the analytic continuation along the path $\gamma_{i, i+1}$, which by definition interchanges $z_{i}$ and $z_{i+1}$ in anticlockwise orientation, and which keeps fixed $z_{1}, \ldots, z_{i-1}, z_{i+2}, \ldots, z_{n}$. Thus, in a more formal notation, $\tau_{i}$ is represented by a braid matrix:

$$
\tau_{i}=\underbrace{1 \otimes \ldots \otimes 1}_{i-1} \otimes R_{i, i+1}^{+} \otimes \underbrace{1 \otimes \ldots \otimes 1}_{n-i-1} .
$$


Similarly,

$$
\tau_{i}^{-1}=\underbrace{1 \otimes \ldots \otimes 1}_{i-1} \otimes R_{i, i+1}^{-} \otimes \underbrace{1 \otimes \ldots \otimes 1}_{n-i-1} .
$$

The generator $\tau_{0}^{2}$ is defined to be the diagonal matrix

$$
\tau_{0}^{2}\left(\left\langle\Omega, \varphi_{1 i i^{*}}\left(z_{1}, \cdot\right) \ldots \Omega\right\rangle\right):=D_{1 i^{*}}^{-} \cdot\left\langle\Omega, \varphi_{1 i i^{*}}\left(z_{1}, \cdot\right) \ldots \Omega\right\rangle
$$

or, more formally,

$$
\tau_{0}^{2}=D^{-} \otimes \underbrace{1 \otimes \ldots \otimes 1}_{n-1} .
$$

In this writing, we can reformulate (4.19)-(4.21) as follows.

Proposition 15. The chiral field theory is defined over $S^{2}$, iff the braid matrices provide us with a linear representation of the modified braid groups $\widetilde{B}_{n+1}\left(S^{2}\right)$, which are defined by the generators $\tau_{0}^{2}, \tau_{1}, \tau_{2}, \ldots, \tau_{n-1}$, and the relations:

a) the usual braid relations on $\mathbb{E}^{2}$ :

$$
\begin{aligned}
\tau_{i} \tau_{j} & =\tau_{j} \tau_{i}, \quad|i-j| \geqq 2 \\
\tau_{i} \tau_{i+1} \tau_{i} & =\tau_{i+1} \tau_{i} \tau_{i+1}
\end{aligned}
$$

b) the extra relations from $S^{2}$ :

$$
\begin{gathered}
\tau_{0}^{2} \tau_{j}=\tau_{j} \tau_{0}^{2}, \quad j \geqq 2 \\
\tau_{1} \tau_{2} \ldots \tau_{n-2}\left(\tau_{n-1}\right)^{2} \tau_{n-2} \ldots \tau_{2} \tau_{1}=\tau_{0}^{2} .
\end{gathered}
$$

Let us remind the reader once more that the very existence of the braid matrices $R^{ \pm}(i j l m)$ implies that they obey (4.32), but that, so far, (4.33) looks like an additional constraint imposed upon them.

Sometimes, symmetries may relate $\left\{\varphi_{i j k}\right\}$ and $\left\{\varphi_{k j^{*} i}\right\}$, and this entails a set of symmetries of the braid matrices. First, recall that $\sum_{k} N_{i j k} N_{k l m}=\sum_{k^{\prime}} N_{i l k^{\prime}} N_{k^{\prime} j m}$. Put $m=j^{*}, l=i$, and we have $\sum_{k} N_{i j k} N_{k j^{*} i}=\sum_{k^{\prime}} N_{i i k^{\prime}} N_{k^{\prime} j j^{*}}$. For any $i$ and $j$ the right-hand side is nonzero (since $k^{\prime}=1$ gives a nonvanishing contribution), and thus there is at least one $k$ with $N_{i j k} \neq 0$ and $N_{k j^{*} i} \neq 0$. Assume that for all $(i, j, k) N_{i j k}=N_{k j^{* i}}$ (which means, in particular, that $\sum_{k^{\prime}} N_{i i k^{\prime}} N_{k^{\prime} j j^{*}}=$ sum of squares). Assume that there is an antilinear, $z$-dependent map $C(z): \mathscr{H}_{j} \rightarrow \mathscr{H}_{j^{*}}$, and a normalization of the chiral vertices such, that

$$
\left(\varphi_{i j k}^{\alpha}(z, \xi)\right)^{*}=\varphi_{k j^{*} i}^{\alpha}\left(\frac{1}{z^{*}}, C(z) \xi\right) .
$$

Clearly, $C(z)$ must satisfy

$$
C(z) \delta_{z}\left(\psi_{r, s}\right)=\delta_{1 / z^{*}}\left(\psi_{r,-s}\right) C(z) .
$$


For Virasoro algebras and $\mathscr{H}_{j^{*}}=\mathscr{H}_{j}$, at least the leading terms of $C(z)$ are easily calculated:

$$
C(z)=z^{*-2 H_{j}} v_{j} \cdot\left\langle, v_{j}\right\rangle+\ldots,
$$

where $v_{j}$ is the primary vector in $\mathscr{H}_{j}$.

Lemma 16. Under the hypothesis (4.34), the braid matrices exhibit the symmetries

$$
\left(R^{+}(i j l m)_{k \alpha \beta}^{k^{\prime} \alpha^{\prime} \beta^{\prime}}\right)^{*}=R^{-}\left(m l^{*} j^{*} i\right)_{k \beta \alpha}^{k^{\prime} \beta^{\prime} \alpha^{\prime}} .
$$

We know already, and we have actually used several times, that the chiral vertices are gauge dependent. I.e., we can rescale

$$
\varphi_{i j k}^{\alpha} \mapsto \lambda_{i j k}^{\alpha} \cdot \varphi_{i j k}^{\alpha},
$$

where $\lambda_{i j k}^{\alpha} \in \mathbb{C}$. So far, we have chosen a specific gauge only for $\varphi_{j j 1}$ and $\varphi_{j 1 j}$ (cf. Sect. 3), if $N_{i j k}$ is arbitrary; and in the case $N_{i j k} \in\{0,1\}$, we can choose a gauge also for $\varphi_{i j k}$, such that (4.15) and (4.25) hold true.

It is easy to check

Lemma 17. The braid relations (4.19)-(4.21) are gauge invariant under

$$
R^{ \pm}(i j l m)_{k \alpha \beta}^{k^{\prime} \alpha^{\prime} \beta^{\prime}} \mapsto \frac{\lambda_{i j k}^{\alpha} \cdot \lambda_{k l m}^{\beta}}{\lambda_{i l k^{\prime}}^{\alpha^{\prime}} \cdot \lambda_{k^{\prime} j m}^{\beta^{\prime}}} \cdot R^{ \pm}(i j l m)_{k \alpha \beta}^{k^{\prime} \alpha^{\prime} \beta^{\prime}}
$$

Remark. Lemma 17 then says that (4.19)-(4.21) do not depend on the normalization chosen for some vertices; as should be clear already from the derivation of these formulae.

\section{Chiral Sectors IV: Chiral Fusion}

The main purpose of this chapter is to show that the chiral vertices $\varphi_{i j k}^{\alpha}(z, \cdot)$ and $\varphi_{k l m}^{\beta}(w, \cdot)$ can be fused. A common interpretation of this fusion is that it represents a short-distance expansion of the product $\varphi_{i j k}^{\alpha}(z, \cdot) \varphi_{k l m}^{\beta}(w, \cdot)$, as $z \rightarrow w$. This shortdistance expansion is a Laurent series in $(z-w)$ around $z=w$, and the coefficients are operator-valued functions which are evaluated at some point close to $z$ and $w$, e.g. at $z$, or $w$, or $\frac{1}{2}(z+w)$. It turns out to be most convenient to choose $\frac{1}{2}(z+w)$, because this allows a treatment which is manifestly symmetric in $z$ and $w$.

As usual, the operator product expansion should be valid whenever inserted into a correlation function, and thus it is natural to expect that the radius of convergence of this infinite series is no larger than the minimum of all distances 2 $\left|z_{i}-\frac{z+w}{2}\right|$, where the points $z_{i}, i=3, \ldots, n$, denote the locations of the $(n-2)$ remaining chiral vertex operators in the $n$-point block. Chiral fusion may also be interpreted as a decomposition of the tensor product $\mathscr{H}_{j} \otimes \mathscr{H}_{l}$, subject to the restrictions that $\mathscr{H}_{k} \subset \mathscr{H}_{l} \otimes \mathscr{H}_{m}$ and $\mathscr{H}_{i} \subset \mathscr{H}_{j} \otimes \mathscr{H}_{k}$, and also $\mathscr{H}_{r} \subset \mathscr{H}_{j} \otimes \mathscr{H}_{l}$ and $\mathscr{H}_{i}$ C $\mathscr{H}_{r} \otimes \mathscr{H}_{m}$.

First, we will study the chiral fusion on the vacuum $\Omega$. Afterwards, the general case will be analyzed. 
Consider the vector $\varphi_{i j k}^{\alpha}(z, \cdot) \varphi_{k k 1}(w, \cdot) \Omega$ in $\mathscr{H}_{\mathscr{L}}$. Since the vacuum is translation invariant, it equals $e^{L-1 \frac{1}{2}(z+w)} \varphi_{i j k}^{\alpha}\left(\frac{z-w}{2}, \cdot\right) \varphi_{k k 1}\left(\frac{w-z}{2}, \cdot\right) \Omega$. Inserting a complete orthonormal set of vectors, $\eta_{i}^{N}$, of $\mathscr{H}_{i}$, and using the normalization (3.17), we get chiral fusion on the vacuum

$$
\begin{aligned}
\varphi_{i j k}^{\alpha}(z, \cdot) \varphi_{k k 1}(w, \cdot) \Omega= & \sum_{N} \varphi_{i i 1}\left(\frac{z+w}{2}, \eta_{i}^{N}\right) \Omega \\
& \cdot\left\langle\eta_{i}^{N}, \varphi_{i j k}^{\alpha}\left(\frac{z-w}{2}, \cdot\right) \varphi_{k k 1}\left(\frac{w-z}{2},\right) \Omega\right\rangle \\
\equiv & {\left[\varphi_{i i 1}(z, w ; \alpha ; \cdot, \cdot)\right] \Omega . }
\end{aligned}
$$

As announced, (5.1) is a power series in $(z-w)$, with $\frac{1}{2}(z+w)$ fixed.

Next, we wish to investigate the operator product $\varphi_{i j k}^{\alpha}(z, \cdot) \varphi_{k l m}^{\beta}(w, \cdot)$. We do this by computing $\varphi_{i j k}^{\alpha}(z, \cdot) \varphi_{k l m}^{\beta}(w, \cdot) \xi_{m}$, where $\xi_{m}$ is an arbitrary vector in $\mathscr{H}_{m}$. By (3.17), $\varphi_{m m 1}\left(0, \xi_{m}\right) \Omega=\xi_{m}$. The idea is, now, to "shift" the pair $\varphi_{i j k}^{\alpha}(z, \cdot) \varphi_{k l m}^{\beta}(w, \cdot)$ in $\varphi_{i j k}^{\alpha}(z, \cdot) \varphi_{k l m}^{\beta}(w, \cdot) \varphi_{m m 1}\left(0, \xi_{m}\right) \Omega$ to the right, in order to make it act on the vacuum $\Omega$; we may then employ the chiral fusion (5.1). Commuting back through $\varphi_{m m 1}\left(0, \xi_{m}\right)$ we will have obtained chiral fusion in its general, symmetric form.

Note that

$$
\begin{gathered}
\mathscr{A}_{\gamma}^{-1} \mathscr{A}_{\gamma}\left(\varphi_{i j k}^{\alpha}(z, \cdot) \varphi_{k l m}^{\beta}(w, \cdot) \varphi_{m m 1}\left(0, \xi_{m}\right) \Omega\right) \\
=\varphi_{i j k}^{\alpha}(z, \cdot) \varphi_{k l m}^{\beta}(w, \cdot) \varphi_{m m 1}\left(0, \xi_{m}\right) \Omega,
\end{gathered}
$$

where $\gamma$ is any path. We apply (5.2) for a path $\gamma$ as indicated in Fig. 8 or in Fig. 9.

Fig. 8

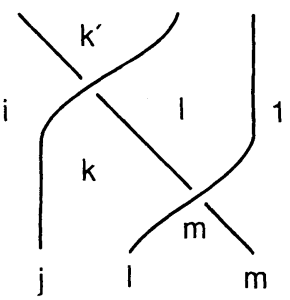

Fig. 9

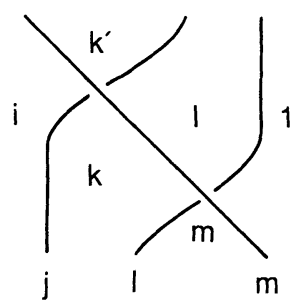

We discuss in detail the analytic continuation in the case where $\gamma$ is given by Fig. 8 ; the case of Fig. 9 is similar. Using (5.1) and the summation convention, we have

$$
\begin{aligned}
& \mathscr{A}_{\gamma}\left(\varphi_{i j k}^{\alpha}(z, \cdot) \varphi_{k l m}^{\beta}(w, \cdot) \varphi_{m m 1}\left(0, \xi_{m}\right) \Omega\right) \\
& \quad=R^{+}(k \ln 1)_{m \beta}^{l \beta^{\prime}} R^{+}(i j m l)_{k \alpha \beta^{\prime}}^{k^{\prime} \alpha^{\prime} \beta^{\prime \prime}} \varphi_{i m k^{\prime}}^{\alpha^{\prime}}\left(z, \xi_{m}\right)\left[\varphi_{k^{\prime} k^{\prime} 1}\left(w, 0 ; \beta^{\prime \prime} ; \cdot, \cdot\right)\right] \Omega .
\end{aligned}
$$

In writing Eq. (5.3) we have assumed, implicitly, that the short-distance expansion in (5.3) makes sense for $\left|z-\frac{w}{2}\right|>\left|\frac{w}{2}\right|$. Furthermore, it is a fundamental assumption that

the fused vector $\varphi_{i m k^{\prime}}^{\alpha^{\prime}}\left(z, \xi_{m}\right)\left[\varphi_{k^{\prime} k^{\prime} 1}\left(w, 0 ; \beta^{\prime \prime} ; \cdot, \cdot\right)\right] \Omega$ can be continued analytically along any path $z \mapsto \mathscr{A}(z), w \mapsto \mathscr{A}(w), 0 \mapsto \mathscr{A}(0)$, as long as $\left|\mathscr{A}(z)-\frac{1}{2}(\mathscr{A}(w)+\mathscr{A}(0))\right|$ 
$>\left|\mathscr{A}(w)-\frac{1}{2}(\mathscr{A}(w)+\mathscr{A}(0))\right|$. Moreover, in these cases the analytic continuation of this vector is given by the analytic continuation of

$$
\varphi_{i m k^{\prime}}^{\alpha^{\prime}}\left(z, \xi_{m}\right), \varphi_{k^{\prime} k^{\prime} 1}\left(\frac{w}{2}, \eta_{k^{\prime}}^{N}\right) \text { and }\left\langle\eta_{k^{\prime}}^{N}, \varphi_{k^{\prime} j l}^{\beta^{\prime \prime}}\left(\frac{w}{2}, \cdot\right) \varphi_{l l 1}\left(-\frac{w}{2}, \cdot\right) \Omega\right\rangle \text {. }
$$

Therefore, the analytic continuation of this vector can again be calculated with the help of braid matrices. This can be understood as follows. The analytic continuation

$\mathscr{A}_{\gamma^{-1}}$ along $\gamma^{-1}$ is split up into a sequence of paths whose union is isotopic to $\gamma^{-1}: \gamma^{-1}=\gamma_{1} \cup \gamma_{2} \cup \gamma_{3}$ (see Fig. 10). $\mathscr{A}_{\gamma_{1}}(w)=y$ and $\mathscr{A}_{\gamma_{1}}(0)=x$, such that $|z|>|y|$ and $y=-x$, which is always possible since $|z|>0$.
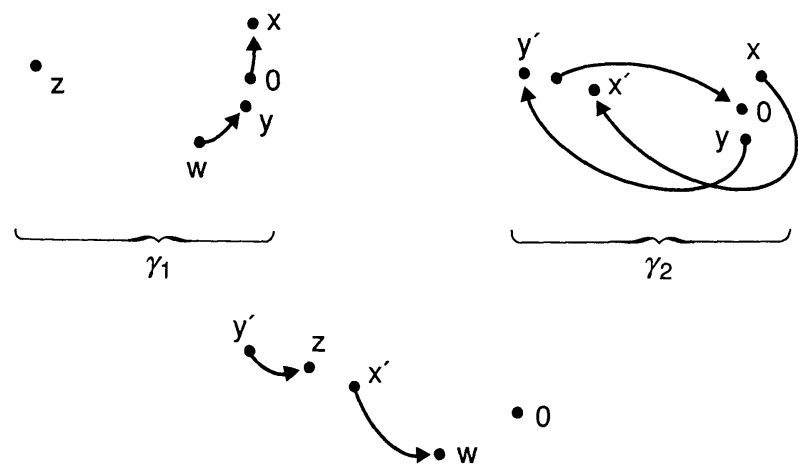

Fig. 10

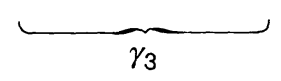

Also, $\mathscr{A}_{\gamma_{2}}$ has to obey the restriction that $\mathscr{A}_{\gamma_{2}}(x)$ does not circle around $\mathscr{A}_{\gamma_{2}}(y)$, because otherwise $\gamma^{-1}$ would not be isotopic to $\gamma_{1} \cup \gamma_{2} \cup \gamma_{3}$. We have $\frac{1}{2}\left(x^{\prime}+y^{\prime}\right)=z$, and in the end we get

$$
\begin{aligned}
& \mathscr{A}_{\gamma^{-1}}\left(\varphi_{i m k^{\prime}}^{\alpha^{\prime}}\left(z, \xi_{m}\right)\left[\varphi_{k^{\prime} k^{\prime} 1}\left(w, 0 ; \beta^{\prime \prime} ; \cdot, \cdot\right)\right] \Omega\right) \\
& \quad=R^{-}\left(i m k^{\prime} 1\right)_{k^{\prime} \alpha^{\prime}}^{m \alpha^{\prime \prime}}\left[\varphi_{i k^{\prime} m}^{\alpha^{\prime \prime}}\left(z, w ; \beta^{\prime \prime} ; \cdot, \cdot\right)\right] \varphi_{m m 1}\left(0, \xi_{m}\right) \Omega,
\end{aligned}
$$

if $\frac{1}{2}|z-w|<\frac{1}{2}|z+w|$. For this range of $z$ and $w$, and upon defining the fusion matrix

$$
F_{i j k l m r}^{\alpha \beta \gamma \beta^{\prime \prime}}:=\sum_{\alpha^{\prime}, \beta^{\prime}} R^{+}(k \operatorname{lm} 1)_{m \beta}^{l \beta^{\prime}} R^{+}(i j m l)_{k \alpha \beta^{\prime}}^{\alpha^{\prime} \beta^{\prime \prime}} R^{-}(i m r 1)_{r \alpha^{\prime}}^{m \gamma},
$$

the combination of (5.2), (5.3), and (5.6) yields

$$
\varphi_{i j k}^{\alpha}(z, \cdot) \varphi_{k l m}^{\beta}(w, \cdot) \xi_{m}=\sum_{r, \gamma, \beta^{\prime \prime}} F_{i j k l m r}^{\alpha \beta \gamma \beta^{\prime \prime}}\left[\varphi_{i r m}^{\gamma}\left(z, w ; \beta^{\prime \prime} ; \cdot, \cdot\right)\right] \xi_{m} .
$$

We remind the reader of the fact that the fusion process could have been performed by the use of the path in Fig. 9, too. This would amount to exchanging all " - " by "+" and vice-versa, in (5.8). We collect our results in

Proposition 18. Chiral vertex operators can be fused; i.e.

$$
\varphi_{i j k}^{\alpha}(z, \cdot) \varphi_{k l m}^{\beta}(w, \cdot)=\sum_{r, \gamma, \beta^{\prime \prime}} F_{i j k l m r}^{\alpha \beta \gamma \beta^{\prime \prime}}\left[\varphi_{i r m}^{\gamma}\left(z, w ; \beta^{\prime \prime} ; \cdot, \cdot\right)\right],
$$

whenever inserted into a n-point block. 
The fusion matrices, $F$, are determined by the braid matrices,

$$
\begin{aligned}
F_{i j k l m r}^{\alpha \beta \gamma \beta^{\prime \prime}} & =\sum_{\alpha^{\prime}, \beta^{\prime}} R^{+}(k \operatorname{lm} 1)_{m \beta}^{l \beta^{\prime}} R^{+}(i j m l)_{k \alpha \beta^{\prime}}^{r \alpha^{\prime} \beta^{\prime \prime}} R^{-}(i m r 1)_{r \alpha^{\prime}}^{m \gamma} \\
& =\sum_{\mu, v} R^{-}(k \operatorname{lm} 1)_{m \beta}^{l v} R^{-}(i j m l)_{k \alpha v}^{r \mu \beta^{\prime \prime}} R^{+}(i m r 1)_{r \mu}^{m \gamma} .
\end{aligned}
$$

We introduce a graphical notation, in order to accommodate braiding and fusion in one picture. The fusion of $\varphi_{i j k}^{\alpha}(z, \cdot)$ and $\varphi_{k l m}^{\beta}(w, \cdot)$ is symbolized in Fig. 11, neatly reflecting the symmetry in $z$ and $w$. On the other hand, the contents of (5.9) and (5.10) is displayed in Fig. 12.

We define the restricted group of gauge transformations to be the group of all rescalings $\varphi_{i j k}^{\alpha} \mapsto \lambda_{i j k}^{\alpha} \cdot \varphi_{i j k}^{\alpha}$, which obey

$$
\lambda_{j j 1}=1, \quad \forall j \in \mathscr{L} .
$$

Thus, the normalization (3.17) is preserved by the restricted gauge transformations. During the calculation of (5.8) we have always employed the gauge (3.17), and so we obtain

\section{Lemma 19.}

a) Under a restricted gauge transformation the fusion matrix transforms as

$$
F_{i j k l m r}^{\alpha \beta \gamma \mu} \mapsto F_{i j k l m r}^{\alpha \beta \gamma \mu} \frac{\lambda_{i j k}^{\alpha} \cdot \lambda_{k l m}^{\beta}}{\lambda_{i r m}^{\gamma} \cdot \lambda_{r j l}^{\mu}} .
$$

b) If (3.17) is not imposed, formulae (5.9) and (5.10) are supplemented by $v_{l l 1}$ and $v_{r r 1}$ in such a way that (5.12) holds true for an arbitrary gauge transformation.

c) Taken together, Eqs. (5.9) and (5.10) imply (4.21); i.e. the chiral field theory is automatically defined over the two-sphere.

Fig. 11

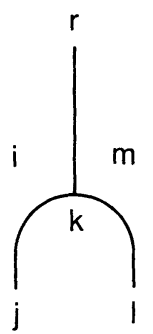

Fig. 12
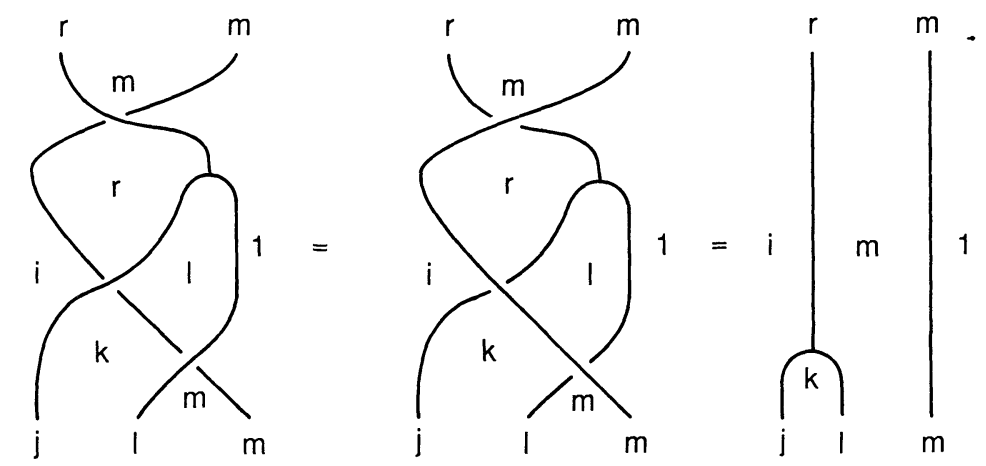
It is remarkable that the existence of braid matrices, plus the compatibility assumption (5.4), mean that the theory is really defined over $S^{2}$.

Apart from the pure braid relations of Chap. 4, new relations for the braid matrices can be derived by examining the consequences of chiral fusion. Such relations are obtained by considering the effect of the combined action of $R$ (analytic continuation) and $F$ (chiral fusion) on products of holomorphic chiral vertices, always keeping in mind the domain of validity of the short distance expansion.

\section{Proposition 20.}

a) The $R$ matrices obey

$$
\sum_{k^{\prime} \alpha^{\prime} \beta^{\prime}} R^{ \pm}(i j l m)_{k \alpha \beta}^{k^{\prime} \alpha^{\prime} \beta^{\prime}} F_{i l k^{\prime} j m r}^{\alpha^{\prime} \beta^{\prime} \gamma \mu}=\sum_{\varrho} R^{ \pm}(r j l 1)_{l \varrho}^{j \mu} F_{i j k l m r}^{\alpha \beta \gamma \varrho}
$$

b) In the notation (5.19), the braid matrices satisfy

c)

$$
\begin{gathered}
\sum_{k^{\prime} \alpha^{\prime} \beta^{\prime}} M^{ \pm}(i j l m)_{k \alpha \beta}^{k^{\prime} \alpha^{\prime} \beta^{\prime}} F_{i j k^{\prime} l m r}^{\alpha^{\prime} \beta^{\prime} \gamma \mu}=D_{r j l}^{\mp} \cdot F_{i j k l m r}^{\alpha \beta \gamma \mu} . \\
\sum_{m^{\prime}, \mu, v, \varrho} R^{ \pm}(k \ln p)_{m \beta \gamma}^{m^{\prime} \mu v} R^{ \pm}\left(i j n m^{\prime}\right)_{k \alpha \mu}^{k^{\prime} \delta^{\prime} \varrho} F_{k^{\prime} j m^{\prime} l p r}^{\varrho v \gamma^{\prime} \beta^{\prime \prime}}=\sum_{\delta} R^{ \pm}(i r n p)_{m \delta \gamma}^{k^{\prime} \delta^{\prime} \gamma^{\prime}} F_{i j k l m r}^{\alpha \beta \delta \beta^{\prime \prime}} .
\end{gathered}
$$

Proof. It may be helpful to first write down the explicit form of $\left[\varphi_{i r m}^{\gamma}\left(z, w ; \beta^{\prime \prime} ; \cdot, \cdot\right)\right]$. We have

$$
\begin{aligned}
{\left[\varphi_{i r m}^{\gamma}\left(z, w ; \beta^{\prime \prime} ; \cdot, \cdot\right)\right]=} & \sum_{N} \varphi_{i r m}^{\gamma}\left(\frac{z+w}{2}, \eta_{r}^{N}\right) \\
& \cdot\left\langle\eta_{r}^{N}, \varphi_{r j l}^{\beta^{\prime \prime}}\left(\frac{z-w}{2}, \cdot\right) \varphi_{l l 1}\left(\frac{w-z}{2}, \cdot\right) \Omega\right\rangle .
\end{aligned}
$$

a) Consider the analytic continuation of the product $\varphi_{i j k}^{\alpha}(z, \cdot) \varphi_{k l m}^{\beta}(w, \cdot)$ along the path $\gamma$ in Fig. 13. For $z$ and $w$ sufficiently close together, we may either fuse the two fields before or after the analytic continuation, and the two expressions thus obtained must agree. The first alternative gives

Fig. 13

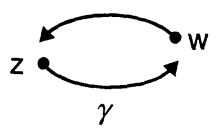

$$
\begin{aligned}
& \mathscr{A}_{\gamma}\left(\varphi_{i j k}^{\alpha}(z, \cdot) \varphi_{k l m}^{\beta}(w, \cdot)\right)=\sum_{r, \gamma, \beta^{\prime \prime}} F_{i j k l m r}^{\alpha \beta \gamma \beta^{\prime \prime}} \sum_{N} \varphi_{i r m}^{\gamma}\left(\frac{z+w}{2}, \eta_{r}^{N}\right) \\
& \cdot \sum_{\sigma} R^{+}(r j l 1)_{l \beta^{\prime \prime}}^{j \sigma}\left\langle\eta_{r}^{N}, \varphi_{r l j}^{\sigma}\left(\frac{z-w}{2}, \cdot\right) \varphi_{j j 1}\left(\frac{w-z}{2}, \cdot\right) \Omega\right\rangle,
\end{aligned}
$$

while the second alternative yields

$$
\begin{aligned}
& \mathscr{A}_{\gamma}\left(\varphi_{i j k}^{\alpha}(z, \cdot) \varphi_{k l m}^{\beta}(w, \cdot)\right)=\sum_{k^{\prime}, \alpha^{\prime}, \beta^{\prime}, \gamma^{\prime}, s, v} R^{+}(i j l m)_{k \alpha \beta}^{k^{\prime} \alpha^{\prime} \beta^{\prime}} F_{i l k^{\prime} j m s}^{\alpha^{\prime} \beta^{\prime} \gamma^{\prime} v} \\
& \cdot \sum_{N} \varphi_{i s m}^{\gamma^{\prime}}\left(\frac{z+w}{2}, \eta_{s}^{N}\right) \cdot\left\langle\eta_{s}^{N}, \varphi_{s l j}^{v}\left(\frac{z-w}{2},\right) \varphi_{j j 1}\left(\frac{w-z}{2}, \cdot\right) \Omega\right\rangle .
\end{aligned}
$$

A little thought shows that $(5.17)=(5.18)$ iff the relation $(5.13)$ is fulfilled. 
In our pictorial language of fused braids, the equality $(5.17)=(5.18)$, or its algebraic counterpart (5.13), have a very simple representation at least if $N_{i j k} \in\{0,1\}$. This is shown in Fig. 14, where we have chosen the gauge (4.14).

Fig. 14
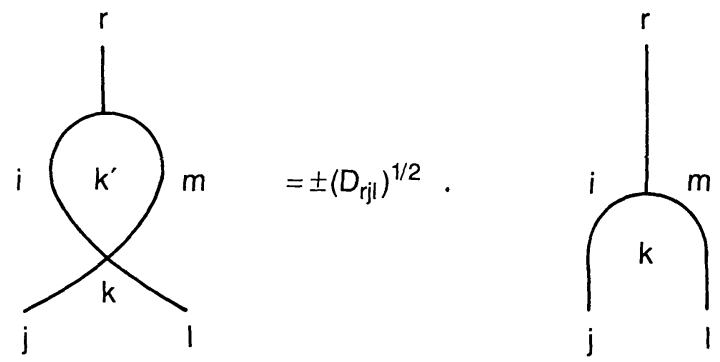

b) Let us iterate once the analytic continuation which led to (5.17) and (5.18). We obtain again a gauge independent algebraic equation, which is necessary and sufficient. Denoting by $M^{ \pm}(i j l m)_{k \alpha \beta}^{k^{\prime \prime} \alpha^{\prime \prime} \beta^{\prime \prime}}$ the monodromy matrix

$$
M^{ \pm}(i j l m)_{k \alpha \beta}^{k^{\prime \prime} \alpha^{\prime \prime} \beta^{\prime \prime}}=\sum_{k^{\prime} \alpha^{\prime} \beta^{\prime}} R^{ \pm}(i j l m)_{k \alpha \beta}^{k^{\prime} \alpha^{\prime} \beta^{\prime}} R^{ \pm}(i l j m)_{k^{\prime} \alpha^{\prime} \beta^{\prime}}^{k^{\prime \prime} \beta^{\prime \prime} \beta^{\prime \prime}},
$$

we find

$$
\sum_{k^{\prime \prime} \alpha^{\prime \prime} \beta^{\prime \prime}} M^{+}(i j l m)_{k \alpha \beta}^{k^{\prime \prime} \alpha^{\prime \prime} \beta^{\prime \prime}} F_{i j k^{\prime \prime l m r}}^{\alpha^{\prime \prime} \beta^{\prime \prime} \gamma \mu}=D_{r j l}^{-} F_{i j k l m r}^{\alpha \beta \gamma \mu}
$$

c) The proof of (5.15) parallels the one of Proposition 18. Anyway, we will offer some comments. Choose points $z, w, u$ which are sufficiently close together, and which obey $|z-w|<|z-u+w-z|,|w-u|<|w-z+u-z|$. We wish to compute the braid-fusion relation which is depicted in Fig. 15.

Fig. 15
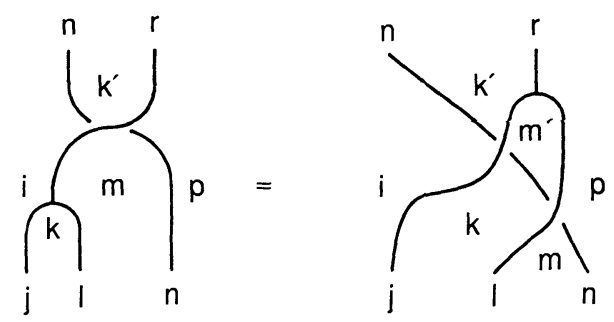

On the one hand, we first fuse $\varphi_{i j k}^{\alpha}(z, \cdot) \varphi_{k l m}^{\beta}(w, \cdot)$, and choose a path $\gamma=\gamma^{\prime} \cup \gamma^{\prime \prime}$ as in Fig. 16 in order to continue analytically the power series $\left[\varphi_{i r m}^{\delta}(z, w\right.$; $\left.\left.\beta^{\prime \prime}, \cdot, \cdot\right)\right] \varphi_{m n p}^{\gamma}(u, \cdot)$.

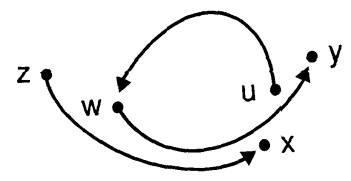

Fig. 16

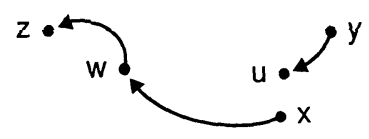

$\gamma^{\prime \prime}$ 
We get

$$
\begin{aligned}
\mathscr{A}_{\gamma}\left(\varphi_{i j k}^{\alpha}(z, \cdot) \varphi_{k l m}^{\beta}(w, \cdot) \varphi_{m n p}^{\gamma}(u, \cdot)\right)= & \sum_{\substack{r, \delta, \beta^{\prime \prime} \\
k^{\prime}, \delta^{\prime}, \gamma^{\prime}}} R^{+}(i r n p)_{m \delta \gamma}^{k^{\prime} \delta^{\prime} \gamma^{\prime}} F_{i j k l m r}^{\alpha \beta \delta \beta^{\prime \prime}} \\
& \cdot \varphi_{i n k^{\prime}}^{\delta^{\prime}}(z, \cdot)\left[\varphi_{k^{\prime} r p}^{\gamma^{\prime}}\left(w, u ; \beta^{\prime \prime}, \cdot, \cdot\right)\right] .
\end{aligned}
$$

On the other hand, if we continue analytically before fusing, we have, because the product $\varphi^{\alpha} \varphi^{\beta} \varphi^{\gamma}$ is analytic at all non-coincidence points, and because $\gamma$ clearly is isotopic to $\gamma^{\prime \prime \prime}$ in Fig. 17,

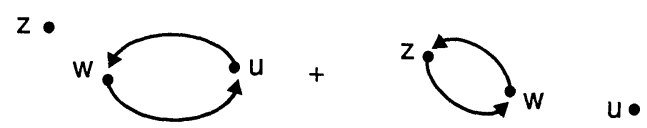

Fig. 17

$$
\gamma^{\prime \prime \prime}
$$

$$
\begin{aligned}
\mathscr{A}_{\gamma}\left(\varphi_{i j k}^{\alpha}(z, \cdot) \varphi_{k l m}^{\beta}(w, \cdot) \varphi_{m n p}^{\gamma}(u, \cdot)\right)= & \sum_{\substack{m^{\prime}, \mu, v, \delta^{\prime} \\
\varrho, k^{\prime}, \gamma^{\prime}, \beta^{\prime}, r}} R^{+}(k \ln p)_{m \beta \gamma}^{m^{\prime} \mu v} R^{+}\left(i j n m^{\prime}\right)_{k \alpha \mu}^{k^{\prime} \delta^{\prime} \varrho} \\
& \cdot F_{k^{\prime} j m^{\prime} l p r}^{o \gamma \beta^{\prime} \beta^{\prime \prime}} \varphi_{i n k^{\prime}}^{\delta^{\prime}}(z, \cdot)\left[\varphi_{k^{\prime} r p}^{\gamma^{\prime}}\left(w, u ; \beta^{\prime \prime}, \cdot, \cdot\right)\right] .
\end{aligned}
$$

A comparison of (5.21) and (5.22) gives (5.15), as a necessary and sufficient condition.

The analysis of the associativity of chiral fusion appears to be less rewarding.

What is not difficult is to realize, that the associativity constrains the possible 4-point blocks. This is briefly explained now. In the domain $|z-w|<|w-u+z-u|$, $|w-u|<|w-z+u-z|$ it is legitimate to perform the fusion processes which are sketched in Fig. 18.

Fig. 18
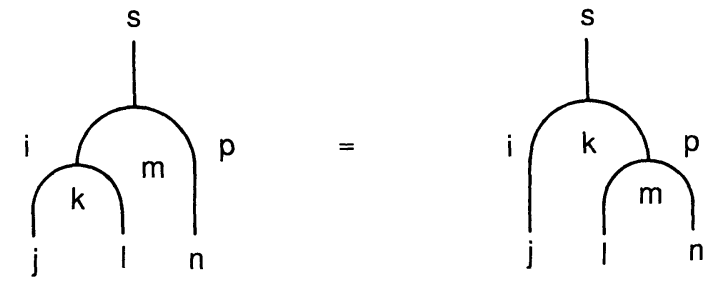

It is useful to note that chiral fusion can be described in various equivalent ways. Skipping all "superfluous" indices, we have e.g. $\varphi(z, \cdot) \varphi(w, \cdot) \Omega=\sum_{N} \varphi\left(w, \eta^{N}\right)$ $\left\langle\eta^{N}, \varphi(z-w, \cdot) \varphi(0, \cdot) \Omega\right\rangle$; and assuming compatibility of chiral fusion (in this form) with analytic continuation we arrive at

$$
\varphi(z, \cdot) \varphi(w, \cdot)=\sum F \sum_{N} \varphi\left(w, \eta^{N}\right)\left\langle\eta^{N}, \varphi(z-w, \cdot) \varphi(0, \cdot) \Omega\right\rangle .
$$

The $F$-matrix here is exactly the same as the $F$-matrix in the symmetric fusion; it is given by (5.9). It appears to be most economic to discuss the associativity of chiral fusion in this "asymmetric" presentation. 
It is easily checked that asymmetric fusion gives

$$
\begin{aligned}
\varphi_{i j k}^{\alpha}(z, & \cdot) \varphi_{k l m}^{\beta}(w, \cdot) \varphi_{m n p}^{\gamma}(u, \cdot)=\sum F_{k l m n p r}^{\beta \gamma \delta \mu} F_{i j k r p s}^{\alpha \delta \varepsilon v} \varphi_{i s p}^{\varepsilon}\left(u, \eta_{s}^{N}\right) \\
& \cdot\left\langle\eta_{s}^{N}, \varphi_{s j r}^{v}(z-u, \cdot) \varphi_{r l n}^{\mu}(w-u, \cdot) \varphi_{n n 1}(0, \cdot) \Omega\right\rangle, \\
= & \sum F_{i j k l m a}^{\alpha \beta \sigma e} F_{i a m n p b}^{\sigma \gamma \varepsilon^{\prime} v^{\prime}} \cdot \varphi_{i b p}^{\varepsilon^{\prime}}\left(u, \eta_{b}^{N}\right)\left\langle\eta_{b}^{N}, \varphi_{b a n}^{v^{\prime}}\left(w-u, \eta_{a}^{N}\right) \varphi_{n n 1}(0, \cdot) \Omega\right\rangle \\
& \cdot\left\langle\eta_{a}^{N}, \varphi_{a j l}^{\varrho}(z-w, \cdot) \varphi_{l l 1}(0, \cdot) \Omega\right\rangle .
\end{aligned}
$$

Therefore, $(5.23)=(5.24)$ for each $\varepsilon=\varepsilon^{\prime}, s=b$, and $\eta_{b}^{N}=\eta_{s}^{N}$ separately. Since $\varphi_{\text {san }}^{v^{\prime}}\left(0, \eta_{a}^{N}\right) \varphi_{n n 1}(u-w, \cdot)=\sum_{v^{\prime \prime}} R^{+}(\operatorname{san} 1)_{n v^{\prime}}^{a v^{\prime \prime}} \varphi_{s n a}^{v^{\prime \prime}}(u-w, \cdot) \varphi_{a a 1}\left(0, \eta_{a}^{N}\right)$, and if $\eta_{s}^{N}$ is an invariant vector $\xi_{s}$, we find (in a sloppy notation)

$$
\begin{aligned}
& \sum_{r, v, \mu} F_{k l m n p r} F_{i j k r p s}\left\langle\xi_{s}, \varphi_{s j r}^{v}(z-u, \cdot) \varphi_{r l n}^{\mu}(w-u, \cdot) \varphi_{n n 1}(0, \cdot) \Omega\right\rangle \\
& \quad=\sum_{a, v^{\prime \prime}, \varrho} F_{i j k l m a} F_{i a m n p s} R^{+}(\operatorname{san} 1)_{n}^{a}\left\langle\xi_{s}, \varphi_{s n a}^{v^{\prime \prime}}(u-w, \cdot) \varphi_{a j l}^{\varrho}(z-w, \cdot) \varphi_{l l 1}(0, \cdot) \Omega\right\rangle .
\end{aligned}
$$

Thus, the associativity connects the 4-point blocks $\left\langle\xi_{s}, \varphi_{s j r}(x, \cdot) \varphi_{r l n}(y, \cdot)\right.$ $\left.\varphi_{n n 1}(0, \cdot) \Omega\right\rangle$ and $\left\langle\xi_{s}, \varphi_{s n a}(0, \cdot) \varphi_{a j l}(x, \cdot) \varphi_{l l 1}(y, \cdot) \Omega\right\rangle$.

We have found a set of algebraic equations which are satisfied by the braid matrices. We are interested in finding out whether they are interrelated and whether they are complete. By definition they would be complete if any combined action of $R$ and $F$ on any $n$-point block does not give new, independent algebraic relations.

With regard to the first problem, a heuristic argument involving braids suggests to compare the YBE to Eq. (5.13). Indeed, it is an easy exercise in algebra to check, that if we project the YBE, (4.20), from the right to the vacuum, i.e. setting $p:=1$ in (4.20), and if we apply the definition of the fusion matrix, (5.9), we obtain precisely Eq. (5.13). Next, it is also evident that (4.2) follows from (4.1). We have already shown that the extra braid relation on $S^{2},(4.21)$, is a consequence of the existence of chiral fusion (5.9), (5.10). Last, (5.14) is just the square of (5.13), by using $\left(4.15^{\prime}\right)$; and the $R^{-}$-version of (5.13) $(5.15)$ follows easily from the $R^{+}$-version by taking inverses.

We have thus obtained

Lemma 21. The braid and fusion relations follow algebraically from

a) $R_{i, i+1}^{+} R_{i, i+1}^{-}=\mathbb{1}$.

b) $R_{i, i+1}^{+} R_{i+1, i+2}^{+} R_{i, i+1}^{+}=R_{i+1, i+2}^{+} R_{i, i+1}^{+} R_{i+1, i+2}^{+}$.

c) $R_{i, i+1}^{+} F_{i, i+1}=F_{i+1, i+2} R_{i, i+1}^{+} R_{i+1, i+2}^{+}$.

(The notation is as in Proposition 15, and the writing of the fusion matrix has been adjusted to that succinct notation.)

Let us turn our attention to the question of completeness. There is a $1-1$ correspondence between the combined action of analytic continuation and fusion on a $n$-point block and its representation in terms of a (fused) coloured braid. If two such actions are isotopic they result in an equation relating braid and fusion matrices. Isotopy means that one (fused) braid can be deformed such as to yield the other braid. The deformation process can be broken down into (generalized) 
Reidemeister-moves. These basic constituents of any deformation are displayed in Figs. 18, 19. However, we recognize immediately that, apart from the associativity of chiral fusion (Fig. 19a), all moves are already encoded by the equations of Lemma 21. We get

Fig. 18

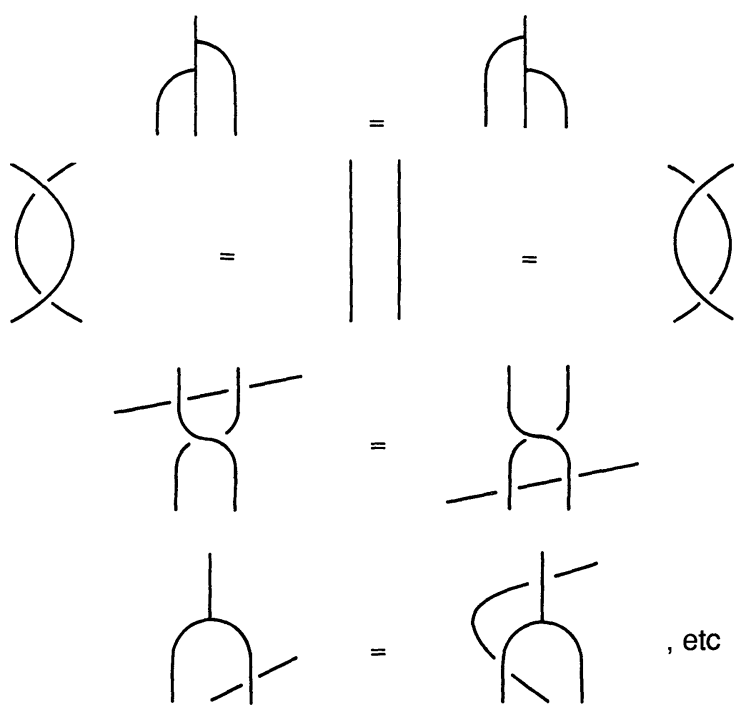

Fig. 19a

\section{Proposition 22.}

a) The equations in Lemma 21, and

b) the associativity equation for chiral fusion are complete, in the sense that no other consistency relations are generated by the combined action of analytic continuation and chiral fusion on n-point blocks.

This result has also been proven in ref. [5].

We wish to point out that, in general, the consistency relation stemming from two isotopic coloured braids can be written down immediately, using the rule that

$$
\begin{aligned}
& \mathrm{j} /\left.\mathrm{k}\right|_{\mathrm{j}} ^{\mathrm{k}^{\prime} / \mathrm{m}} \Leftrightarrow R^{+}(i j l m)_{k \alpha \beta}^{k^{\prime} \alpha^{\prime} \beta^{\prime}} \\
& F_{i j k l m r}^{\alpha \beta \gamma \beta^{\prime \prime}} .
\end{aligned}
$$

The braid-fusion relation (5.15) has important applications in a theory where there is an index, say $2, \in \mathscr{L}$, such that for all $j \in \mathscr{L}$ there is a $m \geqq 2$ with $\mathscr{H}_{j} \subset \mathscr{H}_{2}^{\otimes m}$. Then the knowledge of all $R(\cdot 2 n \cdot)$ and $R(\ldots 1)$ seems to be sufficient to compute the whole set of braid matrices. Actually, we claim that it is enough to know $R(\cdot 22 \cdot), R(\ldots 1)$, in order for a cleverly chosen fusion scheme to provide us with all the braid matrices. This conjecture has been verified explicitly for the case of the minimal models in [6]. 


\section{Chiral Sectors V: Spectrum of the Braid Matrices}

For a given set of indices $\mathrm{ijlm}$ we define the vectors $v(i j l m)^{r \mu \nu}$ with components $v(i j l m)_{k \alpha \beta}^{r \mu \nu}$ by

$$
v(i j l m)_{k \alpha \beta}^{r \mu \nu}:=F_{i j k l m r}^{\alpha \beta \mu \nu} .
$$

The number of these vectors is $\sum_{r} N_{i r m} \cdot N_{r j l}$, which, by (4.7) and (4.8), equals $\sum_{r} N_{i m r} N_{r j l}=\sum_{k} N_{i j k} N_{k l m} \equiv \operatorname{dim}\left(W_{i j l m}\right)$. Using definition (5.9) of the $F$-matrices and the fact that the braid matrices are invertible, it is not hard to verify that the vectors $v(i j l m)^{r \mu v}$ are linearly independent. Now consider Eq. (5.14). It reads, e.g. for " +",

$$
M^{+}(i j l m) v(i j l m)^{r \mu \nu}=D_{r j l}^{-} \cdot v(i j l m)^{r \mu \nu},
$$

thus yielding an eigenvalue equation for the monodromy matrices $M^{+}$and $M^{-}$. Since $M^{+}(i j l m)$ is a $\operatorname{dim}\left(W_{i j l m}\right) \times \operatorname{dim}\left(W_{i j l m}\right)$ square matrix, we get

Lemma 23. The monodromy matrix $\mathrm{M}^{+}($ijlm) has the spectral decomposition

$$
M^{+}(i j l m)=\sum_{r} P_{r} \cdot D_{r j l}^{-},
$$

where $P_{r}$ projects onto the $\left(N_{i m r} \cdot N_{r j l}\right)$-dimensional eigenspace corresponding to the eigenvalue $D_{r j l}^{-}$. The fusion vectors $v(i j l m)^{r \mu v}$ span the image of $P_{r}$.

This result has important applications. Consider Eq. (4.21):

$$
\sum_{k^{\prime} \alpha^{\prime} \beta^{\prime}} R^{+}(i j l m)_{k \alpha \beta}^{k^{\prime} \alpha^{\prime} \beta^{\prime}} D_{k^{\prime} j m}^{-} R^{+}(i l j m)_{k^{\prime} \alpha^{\prime} \beta^{\prime}}^{k^{\prime \prime} \alpha^{\prime \prime} \beta^{\prime \prime}}=\delta_{k k^{\prime \prime}} \delta_{\alpha \alpha^{\prime \prime}} \delta_{\beta \beta^{\prime \prime}} \cdot D_{i j k}^{-}
$$

Since we know the spectral decomposition of $\mathrm{M}^{+}$, one way to extract explicit information from (6.3) and (6.4) is to take the determinant of Eq. (6.4). We obtain

$$
\operatorname{det}\left(M^{+}(i j l m)\right) \cdot \prod_{s}\left(D_{s j m}^{-}\right)^{N_{i l s} N_{s j m}}=\prod_{p}\left(D_{i j p}^{-}\right)^{N_{i j p} N_{p l m}} .
$$

Taking into account (6.3), this can be rewritten as an equation among the conformal dimensions, $H_{q}$, as follows:

$$
\begin{aligned}
& \sum_{r} H_{r}\left(N_{i j r} N_{r l m}+N_{i l r} N_{r j m}+N_{i m r} N_{r j l}\right) \\
& \quad=\left(H_{i}+H_{j}+H_{l}+H_{m}\right) \cdot\left(\sum_{k} N_{i j k} N_{k l m}\right)+n,
\end{aligned}
$$

where $n \in \mathbb{Z}$ is an arbitrary integer.

Considering the determinant of Eq. (6.4) is inspired by Ref. [4], and, indeed, the resulting equation (6.5) coincides with Eq. (7) of Ref. [4]! In other words, we simply need to repeat the steps in Ref. [4] in order to prove that all conformal dimensions, $H_{q}$, are rational numbers. Anyhow, for the convenience of the reader we will review the proof of this statement. We introduce some notation: $d_{i j l m}:=\operatorname{dim}\left(W_{i j l m}\right), d_{i}:=d_{i i i^{*} i}$. Equation (4.6)-(4.9) mean that $d_{i j l m}=d_{i l j m}=d_{i m j l}$, and $d_{i} \geqq 1$. In equation (6.5) we put $i=j=m$, and $i^{*}=l$. In the known models of rational conformal field theory one finds always that $H_{i}=H_{i^{*}}$. At present we do not know whether this is already implied by the property of a triple $(\mathscr{A}, \mathscr{L}, \mathscr{R})$ to be rational. So let us assume that $H_{i}=H_{i *}, \forall i$. From (6.5) we get

$$
4 \cdot d_{i} \cdot H_{i}-\sum_{r} H_{r}\left(2 N_{i i r} N_{r i i^{*}}+N_{i i^{*}} N N_{r i i}\right)=0 \bmod 1
$$


which is a matrix equation

$$
\mathscr{M} \underline{H}=\underline{0} \bmod 1,
$$

where the matrix $\mathscr{M}$ has matrix elements

$$
\begin{aligned}
& \mathscr{M}_{k k}=4 \cdot d_{k}-3 N_{k k k} N_{k k k^{*}} \geqq 1, \\
& \mathscr{M}_{k r}=-2 N_{k k r} N_{r k k^{*}}-N_{k k^{*} r} N_{r k k}, \quad \text { if } r \neq k,
\end{aligned}
$$

and where $\underline{H} \equiv\left(H_{1}, \ldots, H_{n}\right)$ is the vector of the conformal dimensions associated with $\mathscr{H}_{1}, \ldots, \mathscr{H}_{n} \in \mathscr{L}, n=|\mathscr{L}|$.

It follows that

$$
\left|\mathscr{M}_{k k}\right|>\sum_{r \neq k}\left|\mathscr{M}_{k r}\right|
$$

because

$$
4 d_{k}>2 d_{k}+d_{k k^{*} k} \equiv 3 d_{k} .
$$

It has been observed in Ref. [4] that property (6.6) of $\mathscr{M}$ implies, that $\mathscr{M}$ cannot have a trivial eigenvalue, thus $\mathscr{M}$ is invertible. Since $\mathscr{M}$ has integral entries, $\mathscr{M}^{-1}$ is a matrix over $\mathbb{Q}$, therefore $\mathscr{M}^{-1}(\underline{0} \bmod 1)=\underline{H}$ yields

Proposition 24. Let $(\mathscr{A}, \mathscr{L}, \mathscr{R})$ be a rational triple. If

$$
H_{i}=H_{i^{*}}, \text { for all } i \in \mathscr{L} \text {, then } H_{i} \in \mathbb{Q} \text {. }
$$

It would be interesting to explore the full power of Eq. (6.5). In particular, there must be a strong relation between the possible set of conformal dimensions and the fusion rules $N_{i j k}$.

An immediate consequence of Proposition 24 is:

Corollary. All monodromies $M^{ \pm}($ijlm) have the property that

$$
M^{ \pm}(i j l m)^{n}=\left.\mathbb{1}\right|_{W_{i j l m}},
$$

for some $n \in \mathbb{N}$.

\section{Some Relations with Link Invariants and Quantum Groups}

7.1. Link Invariants. In this subsection we intend to indicate briefly how the structural equations which connect the $R$ and $F$ matrices of the holomorphic chiral sector of a conformal field theory can be used for the purpose of constructing link invariants. A more detailed presentation appears in [12]. Motivated by the representation theory of quantum groups, a similar approach has been proposed in [13].

A (n-component) link in $\mathbb{R}^{3}$ consists of $n$ non-intersecting and nonselfintersecting closed curves in $\mathbb{R}^{3}$. If $n=1$, it is called a knot. Two links are equivalent iff they can be deformed continously into each other. We can fix a plane in $\mathbb{R}^{3}$ and project the links onto it. Two links are equivalent iff their projections are equivalent, and the continuous deformations (transferred to the projections) can be viewed as a sequence of those moves which are shown in Fig. 21. These moves 
obviously have much in common with the generalized Reidemeister moves on a (fused) braid. A link invariant is a functional on the set of links, depending only on the equivalence classes of links.

Fig. 21a

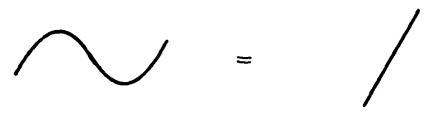

b

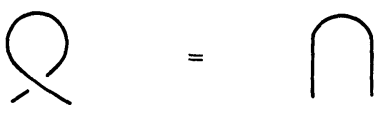

c

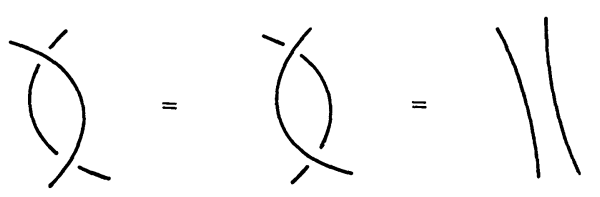

d

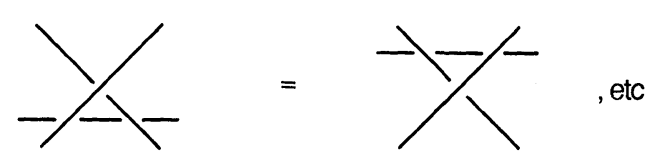

For more information on the theory of knots and links the reader is referred to Refs. $[10,14]$.

We construct a functional, $I(L, O, j), j \in \mathscr{L}$, on the set, $L$, of oriented links in $\mathbb{R}^{3}$ which possess the orientation $O$. (A link is oriented, if each of its strings is so; when drawing pictures, the orientation is indicated by arrows.) The invariant $I(L, O, j)$ is defined relative to a fixed projection plane, $S$, in $\mathbb{R}^{3}$, a fixed, distinguished direction, $\vec{e}$, on $S$, and an index $i \in \mathscr{L}$. The corresponding quantity $I(L, O, j ; S, \vec{e}, i)$ can be shown to be independent of $S, \vec{e}$, and $i$, and because this functional depends but on the equivalence class of the projected oriented links, what is thus obtained is an invariant of oriented links.

We choose a plane, $S$, in $\mathbb{R}^{3}$ and a unit vector $\vec{e} \in S$ which determines the fixed direction. The projection, $L_{S}$, of an oriented link, $L$, on $S$ obtains a marking as follows: a point on $L_{S}$ is marked by - if the tangent vector to $L_{S}$ at this point is orthogonal to $\vec{e}$ (see Fig. 22). Such a marked graph is similar to a vacuum to vacuum Feynman diagram, where annihilation and creation, respectively.

and denote pair Purely for reasons of notational simplicity let us consider a chiral field theory with the properties

a) $N_{i j k} \in\{0,1\}$,

b) $v_{i 1 i}=1$,

c) $j=j^{*}, \forall j \in \mathscr{L}$.

Part b) implies that $R^{ \pm}(\cdot 1 \cdot \cdot)=R^{ \pm}(\cdot \cdot 1 \cdot)=\mathbb{1}$. 
Fig. 22
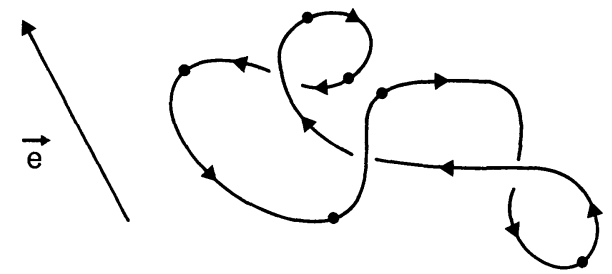

Next, the braid-fusion relation $R^{ \pm}(i j l m)_{k k^{\prime}} F_{k^{\prime} l i l k^{\prime} 1}=R^{\mp}\left(k^{\prime} l j k\right)_{i m} F_{m l k l m 1}$, which can be deduced from (5.15), shows that if for some $l, k^{\prime}, i$ the fusion matrix element $F_{k^{\prime} l i l k^{\prime} 1}=0$, then, for all $k, j, m, R^{\mp}\left(k^{\prime} l j k\right)_{i m} F_{m l k l m 1}=0$. In particular, $F_{l l 1 l l 1}=0$. This, however, would mean that $\sum_{n} a_{n}\left(\varphi_{l l 1}\left(z, \xi_{n}\right) \varphi_{1 l l}\left(z-1, \chi_{n}\right)\right)=0$, for all $z$, where the coefficients $a_{n}$ represent any linear combination of vectors $\xi_{n}$, $\chi_{n}$ such that $\sum_{n} a_{n}\left(\varphi_{r l l}\left(\frac{1}{2}, \xi_{n}\right) \varphi_{l l 1}\left(-\frac{1}{2}, \chi_{n}\right) \Omega\right)=0$ for all $r \neq 1$. Therefore, $F_{l l 1 l l 1}$ cannot possibly vanish, and we see that

d) the numbers $F_{k^{\prime} l i l k^{\prime} 1}$ are nonzero (if they are defined at all).

Now, the link invariant $I(L, O, j) \equiv I(L, O, j ; S, \vec{e}, i)$ is constructed as described in the sequel.

Take the marked diagram $L_{S}$, and transform it into a marked, coloured graph by assigning the index $j$ to its lines, arbitrary labels $k_{l}, k_{l} \in \mathscr{L}$, to its bounded surfaces and the index $i$ to its unique unbounded surface (cf. Fig. 23).

Fig. 23

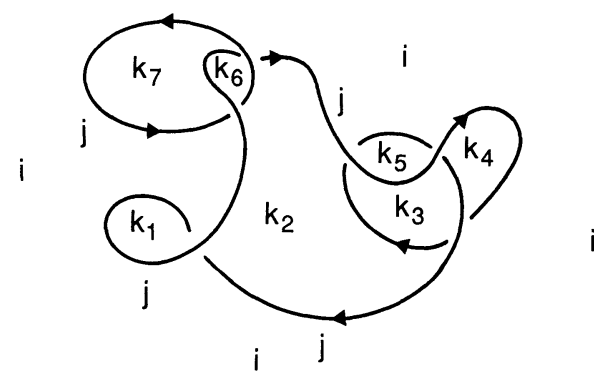

To the building blocks of $L_{S}$ we assign $R$ - and $F$-matrix elements as listed below:
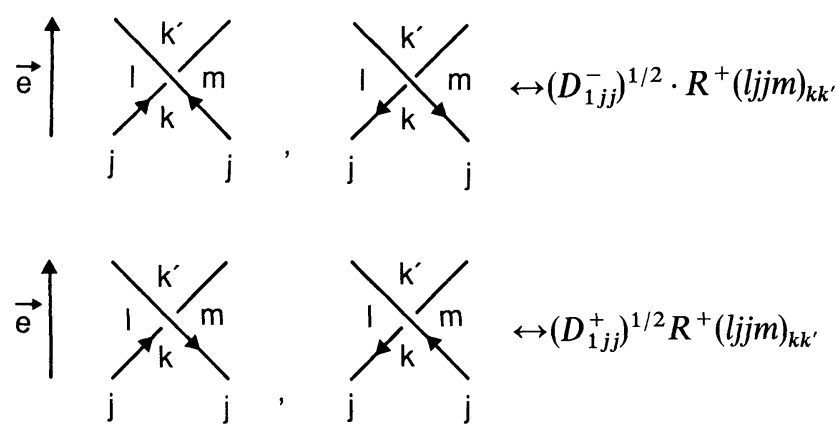

$\leftrightarrow\left(D_{1 j j}^{+}\right)^{1 / 2} R^{+}(l j j m)_{k k^{\prime}}$ 


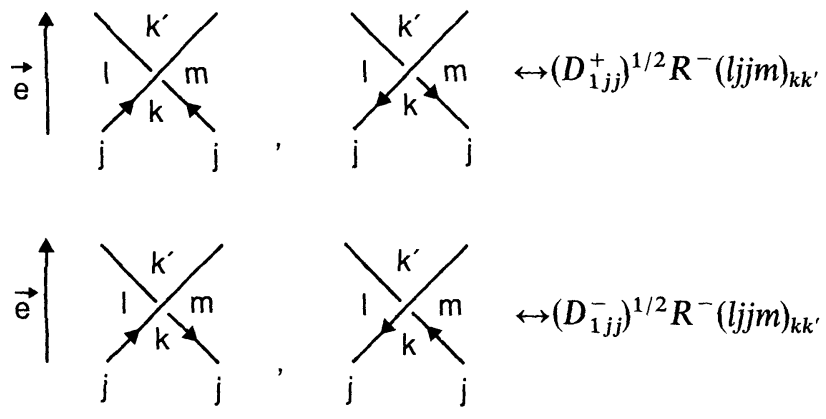

$$
\overrightarrow{\mathrm{e}}
$$

The rules (7.3)-(7.6) and the properties (7.1)-(7.2), together with the structure equations for $R$ and $F$, make it possible to check the relations (summation over internal indices is understood, and $\vec{e}$ is taken to be $\uparrow$ ):
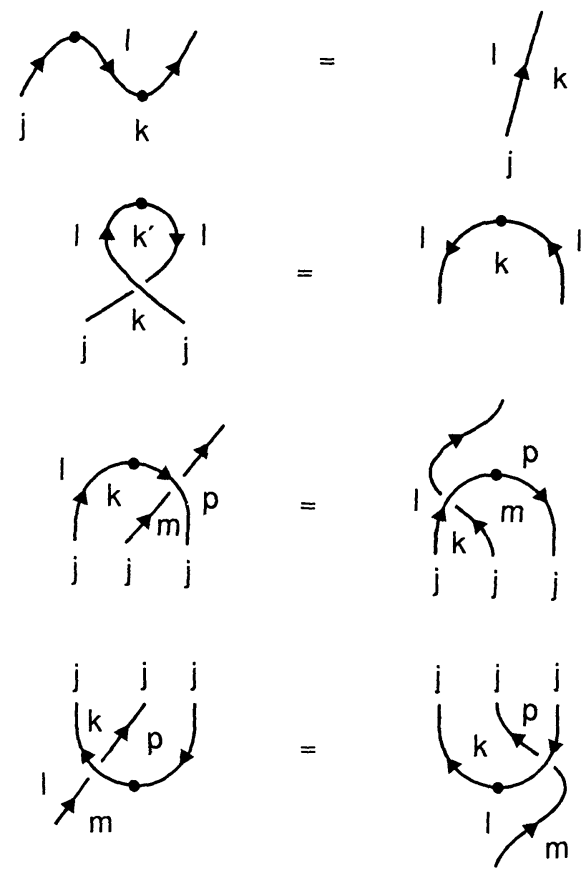

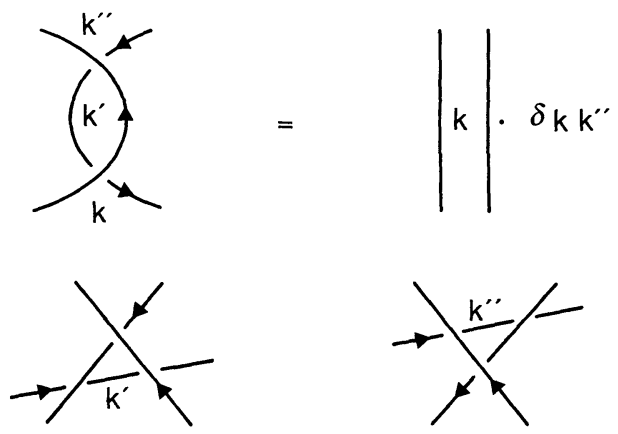

For example, the braid-fusion relation which is relevant to prove (7.8) is Eq. (5.13): $\sum_{k^{\prime}} R^{+}(l j j)_{k k^{\prime}} F_{l j k^{\prime} j l 1}=\left(D_{1 j j}^{-}\right)^{1 / 2} F_{l j k j l 1}$.

The identities (7.7)-(7.12) imply that, for instance,

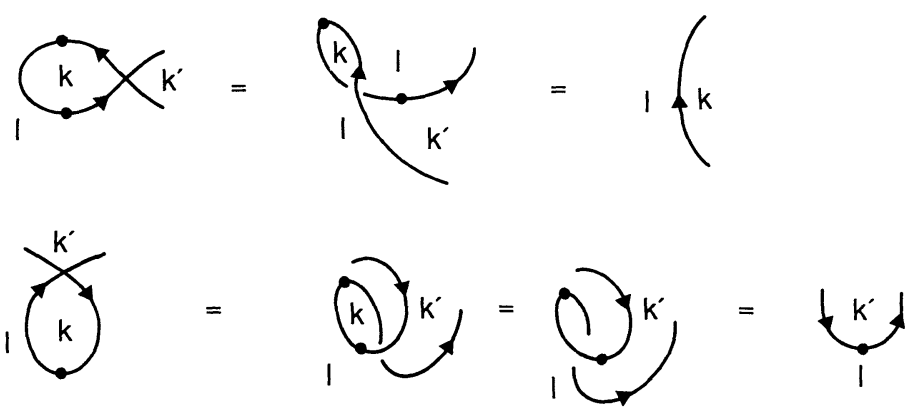

Finally, the link invariant $I(L, O, j)$ is defined by

$$
I(L, O, j ; S, \vec{e}, i):=\sum_{\left\{k_{l}\right\}} \Pi\left(D^{1 / 2} R\right)^{ \pm} \Pi F^{ \pm 1},
$$

where in this symbolic notation the right-hand side of $(7.13) \mathrm{m}(\mathrm{an}$ - the sum over all possible configurations of internal indices, $\left\{k_{l}\right\}$, of the product of all $D^{1 / 2} \cdot R$-matrix elements (arising from crossings) and of all $F$-matrix elements (stemming from fusion or pair creation).

As mentioned earlier, it is not difficult to prove that (7.13) does not depend on $i$, and the same can be shown for $\vec{e}$ and $S$. Furthermore, (7.13) is obviously invariant under all gauge transformations which respect (7.1).

7.2. On Vertex-SOS, Quantum Groups, etc. Suppose that we are given a Hopf algebra, $\mathscr{A}$, with comultiplication $\Delta: \mathscr{A} \rightarrow \mathscr{A} \otimes \mathscr{A}$, and a countable set of irreducible representations of $\mathscr{A}$ on the representation spaces $V_{i}, i \in I$. Assume that, for given $j$, $k$, the multiplicity, $N_{i j k}, N_{i j k}<\infty$, of the representation $i$ in the tensor product representation $j \otimes k$, is non-zero only for a finite set of indices $i$. Furthermore we assume that there are linear operators $R_{j k}: V_{j} \otimes V_{k} \rightarrow V_{k} \otimes V_{j}$ which commute with $\mathscr{A}: \Delta(A) R_{j k}=R_{j k} \Delta(A)$. To simplify the notation in the following, we assume now that $N_{i j k} \in\{0,1\}$. 
Associated to the tensor product decomposition there are projections (which are unique up to a multiplicative constant) $P_{i j k}: V_{j} \otimes V_{k} \rightarrow V_{i}^{2}$, which are characterized by the commutation property $A P_{i j k}=P_{i j k} \Delta(A)$, for all $A \in \mathscr{A}$. This implies that $P_{i k j} R_{j k}=\mu_{i j k} \cdot P_{i j k}$, where $\mu_{i j k} \in \mathbb{C}$. Similar considerations apply to the linear space of those projections which realize the tensor product decomposition $V_{j} \otimes V_{l} \otimes V_{m}$ $\rightarrow V_{i}$. Such projections, denoted by $P_{i j l m}$, obey $A P_{i j l m}=P_{i j l m}(\Delta \otimes 1) \Delta(A), A \in \mathscr{A}$. Obviously, $\left\{P_{i j k} P_{k l m} \mid k \in I\right\}$ and $\left\{P_{i l k^{\prime}} P_{k^{\prime} j m}\left(R_{j l} \otimes 1\right) \mid k^{\prime} \in I\right\}$ and $\left\{P_{i r m} P_{r j l} \mid r \in I\right\}$ form three different bases in the space of projections $P_{i j l m}$. Therefore, there are linear dependences

$$
\begin{gathered}
P_{i j k} P_{k l m}\left(R_{l j} \otimes 1\right)=\sum_{k^{\prime}} r(i j l m)_{k k^{\prime}} P_{i l k^{\prime}} P_{k^{\prime} j m}, \\
P_{i j k} P_{k l m}=\sum_{r} f_{i j k l m r} P_{i r m} P_{r j l},
\end{gathered}
$$

which define the Vertex $\rightarrow$ SOS transformation of the $R$-matrix: $R_{l j} \otimes 1 \rightarrow r(i j l m)_{k k^{\prime}}$, and the "fusion" of projections in (7.15). Assuming the $V_{s}, s \in I$, to be separable Hilbert spaces, we can rewrite (7.14) and (7.15) in terms of vertex operators $\varphi_{i j k}$ which are defined by $\left\langle x_{i}, \varphi_{i j k}\left(x_{j}\right) x_{k}\right\rangle:=\left\langle x_{i}, P_{i j k}\left(x_{j} \otimes x_{k}\right)\right\rangle$, where $x_{s} \in V_{s}$. We obtain

$$
\begin{aligned}
& \varphi_{i j k} \otimes \varphi_{k l m}\left(R_{l j}\left(x_{l} \otimes x_{j}\right)\right)=\sum_{k^{\prime}} r(i j l m)_{k k^{\prime}} \varphi_{i l k^{\prime}}\left(x_{l}\right) \varphi_{k^{\prime} j m}\left(x_{j}\right), \\
& \varphi_{i j k}\left(x_{j}\right) \varphi_{k l m}\left(x_{l}\right)=\sum_{r} f_{i j k l m r} \sum_{N_{r}} \varphi_{i r m}\left(x_{N_{r}}\right)\left\langle x_{N_{r}}, \varphi_{r j l}\left(x_{j}\right) x_{l}\right\rangle,
\end{aligned}
$$

where $\left\{x_{N_{r}} \in V_{r}\right\}$ is an orthonormal basis of $V_{r}$. It is not hard to check that these $r$ and $f$-matrices obey the algebraic relations of the $R^{+}$- and $F$-matrices which were derived in Sects. 4 and 5.

There are known examples of Hopf algebras $\mathscr{A}$ with universal $R$-matrix: the quantum deformations, $U_{q}(g)$, of the universal enveloping algebra of affine or classical Lie-algebras $g[13,22]$. As the deformation parameter, $q$, approaches the rational points on the unit circle, the representation theory for $U_{q}(g)$ becomes rather intricate and in fact has not been solved so far. One expects, however, that a careful examination of this problem shows that there are only a finite number of (finite-dimensional) irreducible representations. Thus, if $q^{m}=1$, the representation theory of rational chiral algebras (as discussed in Sect. 2) is likely to parallel the representation theory for $U_{q}(g)$. In particular, we conjecture that the $s \hat{u}_{k}(n)-\mathrm{WZW}$ theory corresponds to $U_{q}(s l(n)), q=e^{\frac{2 \pi i}{k+n}}$.

We are thus led to conjecture that there is a $1-1$ correspondence among chiral algebras and quantum groups, the SOS-form of the quantum group $R$-matrix, $r(i j l m)_{k k^{\prime}}$, being equal to $R^{+}(i j l m)_{k k^{\prime}}$. In particular, the multiplicities $N_{i j k}$ for the chiral algebra equal those of some quantum group.

Let us mention that the Vertex-SOS transformation of quantum group $R$-matrices has been studied also in Ref. [23].

In the remainder of this section we show that the above conjecture (on the relation between rational conformal field theories and quantum groups) and the Vertex-SOS transformation of the quantum group $R$-matrices permit the cons-

\footnotetext{
${ }^{2}$ Notice that we do not mean that the tensor product $V_{j} \otimes V_{k}$ is completely reducible in terms of $V_{s}$, $s \in I$
} 
truction of new vertex operators, $V_{j j^{\prime}}(z, \cdot, \cdot)$, whose commutation relations are described by the Vertex-version of the $R$-matrices. ${ }^{3}$

Thus, we assume that for a given RCFTh with vertex operators ${ }^{4} \varphi_{i j k}(z, \cdot)$ and corresponding $R$-matrices $R^{+}(i j l m)_{k p}$ and multiplicities $N_{i j k} \in\{0,1\}$ there is a quantum group, $U_{q}$, with vertex operators $\psi_{i j k}(\cdot)$, with SOS $R$-matrices $r(i j l m)_{k p}$ $=R^{+}(i j l m)_{k p}$ and which has the same $N_{i j k}$. Assume that we can find coefficients $D_{i j k}^{i^{\prime} j^{\prime} k^{\prime}} \in \mathbb{C}, i, j, k, i^{\prime}, j^{\prime}, k^{\prime} \in I$, such that (cf. (8.10))

$$
\sum_{k, k^{\prime}} R^{+}(i j l m)_{k p} R^{-}\left(i^{\prime} j^{\prime} l^{\prime} m^{\prime}\right)_{k^{\prime} p^{\prime}} D_{i j k}^{i^{\prime} j^{\prime} k^{\prime}} D_{k l m}^{k^{\prime} l^{\prime} m^{\prime}}=D_{i l p}^{i^{\prime} l^{\prime} p^{\prime}} D_{p j m}^{p^{\prime} j^{\prime} m^{\prime}} .
$$

We note that (cf. Sect. 8) if $j=j^{*}, \forall j \in I$, and if there is a gauge such that $R^{+}(i j l m)_{k p}$ $=R^{+}(m l j i)_{k p}$, then a solution of $(7.18)$ is given by

$$
D_{i j k}^{i^{\prime} j^{\prime} k^{\prime}}=\delta_{i i^{\prime}} \delta_{j j^{\prime}} \delta_{k k^{\prime}} \cdot\left(F_{i j k j i 1}\right)^{-1} .
$$

We notice also that (7.19) is indeed a solution for the minimal models and for $s \hat{u}_{k}(2)$ current-algebra. $\mathrm{\vdash} U \mathrm{t}_{\mathrm{t}}{ }^{\prime}{ }_{j}$ and $x \in V_{j^{\prime}}$, where $V_{j^{\prime}}$ is the quantum group representation corresponding to $\mathscr{H}_{j}$, we define

$$
V_{j j^{\prime}}(z, \xi, x):=\sum_{\substack{i i^{\prime} \\ k k^{\prime}}} D_{i j k}^{i^{\prime} j^{\prime} k^{\prime}} \varphi_{i j k}(z, \xi) \psi_{i^{\prime} j^{\prime} k^{\prime}}(x)
$$

Computing the statistics of the vertices $V_{j j^{\prime}}$, we get $(\gamma$ is a path exchanging $z$ and $w$ in positive orientation)

$$
\begin{aligned}
& \mathscr{A}_{\gamma}\left(V_{j j^{\prime}}(z, \cdot, \cdot) V_{l l^{\prime}}(w, \cdot, \cdot)\right) \\
& \quad=\sum_{\substack{i i^{\prime} \\
m m^{\prime} \\
p k^{\prime}}}\left(\sum_{k} D_{i j k}^{i^{\prime} j^{\prime} k^{\prime}} D_{k l m}^{k^{\prime} l^{\prime} m^{\prime}} R^{+}(i j l m)_{k p}\right) \psi_{i^{\prime} j^{\prime} k^{\prime}}(\cdot) \psi_{k^{\prime} l^{\prime} m^{\prime}}(\cdot) \varphi_{i l p}(z, \cdot) \varphi_{p j m}(w, \cdot),
\end{aligned}
$$

which, using (7.18), equals

$$
=\sum_{\substack{i i^{\prime} \\ m m^{\prime} \\ p p^{\prime}}}\left(\sum_{k^{\prime}} R^{+}\left(i^{\prime} l^{\prime} j^{\prime} m^{\prime}\right)_{p^{\prime} k^{\prime}} \psi_{i^{\prime} j^{\prime} k^{\prime}}(\cdot) \psi_{k^{\prime} l^{\prime} m^{\prime}}(\cdot)\right) D_{i l p}^{i_{i}^{\prime} l^{\prime} p^{\prime}} D_{p j m}^{p^{\prime} j^{\prime} m^{\prime}} \varphi_{i l p}(z, \cdot) \varphi_{p j m}(w, \cdot) .
$$

Since $R^{+}=r$, the Vertex-SOS transformation (7.16) can be applied. This gives

$$
=\sum_{\substack{i i^{\prime} \\ p p^{\prime} \\ m m^{\prime}}} D_{i l p}^{i^{\prime} l^{\prime} p^{\prime}} D_{p j m}^{p^{\prime} j^{\prime} m^{\prime}} \varphi_{i l p}(z, \cdot) \varphi_{p j m}(w, \cdot) \psi_{i^{\prime} l^{\prime} p^{\prime}} \otimes \psi_{p^{\prime} j^{\prime} m^{\prime}}\left(R_{j^{\prime} l^{\prime}}(\cdot \otimes \cdot)\right) .
$$

The result is written most easily using an orthonormal basis $e_{\alpha}^{j^{\prime}} \otimes e_{\beta}^{l^{\prime}} \in V_{j^{\prime}} \otimes V_{l^{\prime}}$. We find

$$
\mathscr{A}_{\gamma}\left(V_{j j^{\prime}}\left(z, \cdot, e_{\alpha}^{j^{\prime}}\right) V_{l l^{\prime}}\left(w, \cdot, e_{\beta}^{l^{\prime}}\right)\right)=\sum_{\gamma \delta}\left(R_{j^{\prime} l^{\prime}}\right)_{\gamma \delta, \alpha \beta} V_{l l^{\prime}}\left(z, \cdot, e_{\gamma}^{l^{\prime}}\right) V_{j j^{\prime}}\left(w, \cdot, e_{\delta}^{j^{\prime}}\right),
$$

where $\left\langle e_{\gamma}^{l^{\prime}} \otimes e_{\delta}^{j^{\prime}}, R_{j^{\prime} l^{\prime}} e_{\alpha}^{j^{\prime}} \otimes e_{\beta}^{j^{\prime}}\right\rangle \equiv\left(R_{j^{\prime} l^{\prime}}\right)_{\gamma \delta, \alpha \beta}$.

\footnotetext{
${ }^{3}$ The construction of the operators $V_{j j^{\prime}}$ is rather similar to the construction of local fields in terms of a holomorphic and an antiholomorphic chiral field theory

${ }^{4}$ In order to keep the notation as simple as possible we assume here that the multiplicities $N_{i j k}$ are either zero or one
} 


\section{Conformal Field Theory of Local Bose Fields over $S^{2}$}

Suppose that we are given two rational chiral field theories, $(\mathscr{A}, \mathscr{L}, \mathscr{R})$ and $(\overline{\mathscr{A}}, \overline{\mathscr{L}}, \overline{\mathscr{R}})$ for short. We wish to combine them, or rather their chiral vertices, in such a way that they provide us with a set of relatively local bose fields over $S^{2}$. This combination process, called local fusion, is not some a priori and uniquely prescribed method, but rather we propose one specific approach which is motivated by the holomorphic factorization properties which a conformal field theory should exhibit. Let us add that two chiral sectors can successfully be subjected to this kind of local fusion iff their braid matrices obey the algebraic relation (8.10). For example, if for some $j \in \mathscr{L}$ there is no $\bar{j} \in \overline{\mathscr{L}}$ with $\left(H_{j}-H_{\bar{j}}\right) \in \mathbb{Z}$, then this local fusion is impossible. It is an important, but as yet unsolved, problem to give explicit criteria which would guarantee that $(\mathscr{A}, \mathscr{L}, \mathscr{R})$ and $(\overline{\mathscr{A}}, \overline{\mathscr{L}}, \overline{\mathscr{R}})$ can be fused.

Let $z$ and $\bar{z}$ be independent complex variables, $z \in \mathbb{C}, \bar{z} \in \mathbb{C}$. The chiral vertices, $\varphi_{i j k}^{\alpha}(z, \cdot)$, of the holomorphic chiral sector $(\mathscr{A}, \mathscr{L}, \mathscr{R})$, and those of the antiholomorphic sector $(\overline{\mathscr{A}}, \overline{\mathscr{L}}, \overline{\mathscr{R}})$, i.e. $\varphi_{i j \bar{\alpha}}^{\bar{\alpha}}(\bar{z}, \cdot)$, are combined into local fields, according to the Ansatz:

$$
\phi_{j \bar{j}}^{\alpha}(z \bar{z}, \cdot):=\sum_{i, \bar{i}, k, \bar{k}, \beta, \bar{\beta}} \tilde{C}\left((\overline{j j \alpha})_{i k \beta}^{i \bar{k} \bar{\beta}} \varphi_{i j k}^{\beta}(z, \cdot) \varphi_{i \bar{j} \bar{k}}^{\bar{\beta}}(\bar{z}, \cdot) .\right.
$$

Let us explain the meaning of this definition. First, the fields $\phi_{J}^{\alpha}(z \bar{z}, \cdot) \equiv \phi_{j j}^{\alpha}(z \bar{z}, \cdot)$ are operator-valued fields on $S^{2} \times S^{2}$, which furnish maps

$$
\phi_{J}^{\alpha}: \mathscr{H}_{j} \otimes \mathscr{H}_{\mathscr{L}} \otimes \mathscr{H}_{j} \otimes \mathscr{H}_{\mathscr{L}} \rightarrow \mathscr{H}_{\mathscr{L}} \otimes \mathscr{H}_{\bar{L}} .
$$

The objects $\widetilde{C}(j \bar{j} \alpha)_{i k \beta}^{i k \bar{\beta}}$ are $\mathbb{C}$-number coefficients which determine the bilinear combination of the chiral vertices. The index $\alpha$ on $\phi_{J}^{\alpha}$ is meant to distinguish among linearly independent local fields, i.e. we require that the fusion coefficients $\widetilde{C}\left(j \bar{j} \alpha_{p}\right), p=1, \ldots, n$, encode fields which satisfy

$$
\sum_{p=1}^{n} \lambda_{p} \phi_{J}^{\alpha_{p}}(z \bar{z}, \cdot)=0 \Leftrightarrow \lambda_{p}=0, \forall p
$$

Clearly, the maximal number of linearly independent combinations $\phi_{J}^{\alpha}$, for fixed $J$, is finite.

Finally, postulating bose field locality says that on the Euclidean section of $S^{2}$ $\times S^{2}$, i.e. at the points $\left(z, \bar{z}=z^{*}\right) \in S^{2} \times S^{2}$, the fields $\phi_{J}^{\alpha}$ form a set of relatively local bose fields.

It should be stressed that requiring this trivial commutation relation among all the fields $\phi_{J}^{\alpha}$ does not indicate a limit beyond which one cannot reach using this formalism. In fact, any representation of the braid group other than the trivial one would have been wonderful, too, apart from the calculational complications which we had wanted to evade.

Let us take a break for a moment and think about the question what the "physical" Hilbert space is. By definition, this is the Hilbert space, $\hat{\mathscr{H}}$, which is associated to the local conformal field theory $(\mathscr{A} \otimes \overline{\mathscr{A}}, \widetilde{C})$. If the fusion of the chiral 
sectors is sufficiently uncomplicated then it is easy to describe $\hat{\mathscr{H}}$ explicitly. For, supposing that

a) per index $J \hat{=}(j, \bar{j})$ there is at most one local field;

b) for each field $\phi_{J}$ the fusion coefficients $\widetilde{C}(j \bar{j})_{j 1}^{\bar{j}}$ and $\tilde{C}(j \bar{j})_{1 j^{*}}^{\bar{i} j^{*}}$ are nonzero; (8.4)

c) let $\hat{\mathscr{L}}$ be the index set of the local fields, thus here $\mathscr{\mathscr { L }} \subset \mathscr{L} \times \overline{\mathscr{L}}$.

If $\widetilde{C}(j \bar{j})_{i k}^{i \bar{k}} \neq 0$, then $(i, \bar{i}) \in \hat{\mathscr{L}}$ and $(k, \bar{k}) \in \hat{\mathscr{L}}$;

then the domain of definition (as well as the range) of the local fields is

$$
\hat{\mathscr{H}}:=\underset{(j, \hat{j}) \in \hat{\mathscr{L}}}{\oplus}\left(\mathscr{H}_{j} \otimes \mathscr{H}_{j}\right) .
$$

Clearly, $\quad \hat{\mathscr{H}} \subset\left(\mathscr{H}_{\mathscr{L}} \otimes \mathscr{H}_{\overline{\mathscr{L}}}\right), \quad\left\{\lim _{z \rightarrow 0} \lim _{\bar{z} \rightarrow 0} \phi_{J}(z \bar{z}, \xi \bar{\xi}) \Omega \mid \xi \in \mathscr{H}_{j}, \quad \bar{\xi} \in \mathscr{H}_{\bar{j}} ; \quad \Omega \equiv \Omega_{\mathscr{L}} \otimes \Omega_{\overline{\mathscr{L}}}\right\}$ $=\mathscr{H}_{j} \otimes \mathscr{H}_{\hat{j}}$. Also, $\phi_{J}$ maps $\hat{\mathscr{H}}$ into $\hat{\mathscr{H}}$, and the two point function $\left\langle\Omega, \phi_{J^{*}}(z \bar{z}, \cdot) \phi_{J}(w \bar{w}, \cdot) \Omega\right\rangle$ is nontrivial. Most notably, two local sectors $\mathscr{H}_{i} \otimes \mathscr{H}_{\bar{i}}$ and $\mathscr{H}_{j} \otimes \mathscr{H}_{j}$ are orthogonal iff there are corresponding, linearly independent fields $\phi_{I}$ and $\phi_{J}$ which generate them as $\phi_{I}: \mathscr{H}_{1} \otimes \mathscr{H}_{\overline{1}} \rightarrow \mathscr{H}_{i} \otimes \mathscr{H}_{\bar{i}}, \phi_{J}: \mathscr{H}_{1} \otimes \mathscr{H}_{\overline{1}} \rightarrow \mathscr{H}_{j} \otimes \mathscr{H}_{\bar{j}}$. We conclude this discussion by noting that among the examples, where the structure (8.4) is present, are the $\left(A_{p^{\prime}-1}, A_{p-1}\right)$ and $\left(D_{2 \varrho+1}, A_{p-1}\right)$ series of the Cappelli-Itzykson-Zuber classification of modular invariant minimal models [15].

In general, (8.4) is not fulfilled. The reconstruction of $\hat{\mathscr{H}}$ in these cases proceeds by an application of Osterwalder-Schrader reconstruction. We choose a basis of $L_{0}$-eigenstates, $\xi_{i}^{N}$, in $\mathscr{H}_{i}$, and put $\bar{z}=z^{*}$. On the space, $\mathscr{I}_{1}$, of finite sequences of Schwartz test functions over the unit disk one defines the non-negative inner product

$$
\langle f, g\rangle_{1}:=\sum_{n, m} \sum_{\mu, v} \int \prod_{i=1}^{n} d^{2} z_{i} \prod_{j=1}^{m} d^{2} w_{j} G_{\boldsymbol{\mu}, \boldsymbol{v}}(\mathbf{z}, \mathbf{w}) \cdot f_{\boldsymbol{\mu}}(\mathbf{z})^{*} g_{\mathbf{v}}(\mathbf{w}) \text {. }
$$

Here, $G_{\mu, v}(\mathbf{z}, \mathbf{w}) \equiv\left\langle\phi_{J_{1}}^{\alpha_{1}}\left(z_{1} z_{1}^{*}, \xi_{j_{1}}^{N_{j_{1}}} \xi_{j_{1}}^{\bar{N}_{j_{1}}}\right) \ldots \phi_{J_{n}}^{\alpha_{n}}(\ldots) \Omega, \phi_{I_{1}}^{\beta_{1}}\left(w_{1} w_{1}^{*}, \xi_{i_{1}}^{N_{i_{1}} \xi_{i_{1}}^{N_{i_{1}}}} \ldots \Phi_{I_{m}}^{\beta_{m}}(\ldots) \Omega\right\rangle\right.$, and $\boldsymbol{\mu} \equiv\left(\mu_{1}, \ldots, \mu_{n}\right), \boldsymbol{v} \equiv\left(v_{1}, \ldots, v_{m}\right)$, where $\mu_{1} \equiv\left(J_{1}, \alpha_{1}, N_{j_{1}}, \bar{N}_{j_{1}}\right), \ldots, v_{1} \equiv\left(I_{1}, \beta_{1}, N_{i_{1}}\right.$, $\left.\bar{N}_{\bar{i}_{1}}\right), \ldots$. The test functions $f_{\boldsymbol{\mu}}(\mathbf{z}) \equiv f_{\boldsymbol{\mu}}\left(z_{1}, \ldots, z_{n}\right)$ and $g_{\mathbf{v}}(\mathbf{w})$ have support in $\left(D_{1}\right)^{n} \backslash$ diagonal and $\left(D_{1}\right)^{m} \backslash$ diagonal, $D_{1} \equiv$ unit disk. Finally, $f \in \mathscr{I}_{1}$ stands for a sequence of test functions $f=\left(f_{\boldsymbol{\mu}^{(1)}}, f_{\boldsymbol{\mu}^{(2)}}, \ldots\right)$, where apart from a finite number of them all the $f_{\mu^{(k)}}$ are zero.

Denoting by $\mathscr{N}_{1}$ the space of zero norm vectors in $\mathscr{J}_{1}$, one is led to define

$$
\hat{\mathscr{H}}:=\left(\mathscr{I}_{1} / \mathscr{N}_{1}\right)^{-} .
$$

There is an action of the chiral algebras $\mathscr{A} \otimes \overline{\mathscr{A}}$ on $\hat{\mathscr{H}}$, defined by

$$
\langle f, A \otimes \bar{A} g\rangle_{1}:=\sum_{n, m} \sum_{\mu, v} \int G_{\mu, v}^{A \otimes \bar{A}}(\mathbf{z}, \mathbf{w}) \cdot f_{\mu}(\mathbf{z}) * g_{v}(\mathbf{w}),
$$

where $G_{\mu, v}^{A \otimes \bar{A}}(\mathbf{z}, \mathbf{w})=\left\langle\phi_{J_{1}}^{\alpha_{1}}(\ldots) \ldots \Omega, A \otimes \bar{A} \phi_{I_{1}}^{\beta_{1}}(\ldots) \ldots \Omega\right\rangle$. In particular, $L_{-1} g_{\mathbf{v}}(\mathbf{w})$ $=-\sum_{j=1}^{m} \frac{\partial}{\partial w_{j}} g_{v}(\mathbf{w}) ;$ and the vectors $g \equiv g_{v}\left(\right.$ with $v=\left(J, \alpha, N_{j}, \bar{N}_{j}\right)$, and $\xi_{j}^{N_{j}}$ and $\xi_{j}^{\bar{N}_{j}}$ invariant vectors) are invariant if $g_{v}(w)=\delta^{(2)}(w, 0) \cdot g_{v}(w)$. We have not worked out all the details, but it is expected that

$$
\hat{\mathscr{H}} \simeq \underset{(j, \bar{j}, \alpha) \in \overline{\mathscr{L}}}{\oplus}\left(\mathscr{H}_{j} \otimes \mathscr{H}_{\mathrm{j}}\right)
$$


After this digression we resume the discussion of the general properties of a local conformal field theory over $S^{2}$. We prove

Proposition 25. Locality (as bose fields) is equivalent to the following equation for the fusion coefficients:

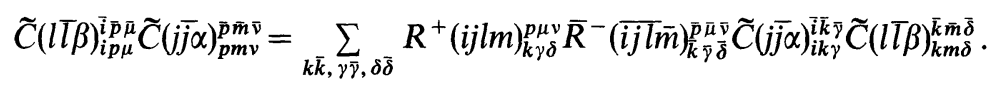

Proof. Take euclidean points $z, \bar{z}=z^{*}$ and $w, \bar{w}=w^{*}$, and consider the analytic continuation of $\phi_{J}^{\alpha}(z \bar{z}, \cdot) \phi_{L}^{\beta}(w \bar{w}, \cdot)$ along the euclidean path $\gamma_{E}$ which exchanges $z$ and $w$ in anticlockwise orientation (Fig. 24). Requiring bose field locality is equivalent
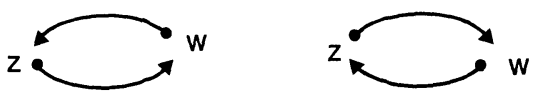

Fig. 24

$\gamma_{E}$

to demand that $\mathscr{A}_{\gamma}\left(\phi_{J}^{\alpha}(z \bar{z}, \cdot) \phi_{L}^{\beta}(w \bar{w}, \cdot)\right)=\phi_{L}^{\beta}(z \bar{z}, \cdot) \phi_{J}^{\alpha}(w \bar{w}, \cdot)$. Writing out the former and latter operator products in terms of chiral vertices we see that $(8.10)$ is the necessary and sufficient condition to guarantee locality.

If we iterate $(8.10)$ once, we obtain the eigenvalue equation

$$
v(J \alpha, L \beta, i m)=v(J \alpha, L \beta, i m)\left(M^{+}(i j l m) \otimes \bar{M}^{-}(\overline{i j l} \bar{m})\right),
$$

where the vectors $v(J \alpha, L \beta, i m)$ have components

$$
v(J \alpha, L \beta, i m)_{p \mu \nu}^{\bar{p} \bar{\mu} \bar{\nu}} \equiv \widetilde{C}(j \bar{j} \alpha)_{i p \mu}^{\bar{i} \bar{p} \bar{\mu}} \widetilde{C}(l T \beta)_{p m \nu}^{\bar{p} \bar{m} \bar{\nu}} .
$$

We assume henceforth that

$$
\phi_{J}^{\alpha} \neq 0 \Rightarrow \widetilde{C}(j \overline{j \alpha})_{j 1}^{j \overline{1}} \neq 0 .
$$

(The reverse of (8.13) is, of course, always true.)

Now putting $m=1, \bar{m}=\overline{1}$ in (8.10) reveals that

$$
\widetilde{C}(l T \beta)_{i j \mu}^{\bar{i} \bar{\mu}} \widetilde{C}(j \overline{j \alpha})_{j 1}^{\bar{j} \overline{1}}=\sum_{\gamma \bar{\gamma}} R^{+}(i j l 1)_{l \gamma}^{j \mu} \bar{R}^{-}(\overline{i j l 1})_{\bar{l} \bar{\gamma}}^{\bar{j} \tilde{C}} \widetilde{C}(\overline{j \alpha})_{i l \gamma}^{\bar{i} \bar{\gamma} \tilde{C}} \widetilde{C}(l \bar{l} \beta)_{l 1}^{\bar{I} \overline{1}} .
$$

In particular, if $\phi_{J} \neq 0$ and $\phi_{L}^{\cdot} \neq 0$, then $\tilde{C}(j \bar{j} \cdot)_{i j}^{i \bar{i} \cdot}=0$ iff $\widetilde{C}(l T \cdot)_{i j}^{i \bar{j}}=0$.

Equation (8.14) has an important application. Namely, it allows us to formulate the short-distance expansion among the local fields in a transparent way. This is what we are going to show next.

Take two local fields, $\phi_{J}^{\alpha}$ and $\phi_{L}^{\beta}$. Writing them in terms of the chiral constituents, and employing chiral fusion, yields

$$
\begin{aligned}
& \phi_{J}^{\alpha}(z \bar{z}, \cdot) \phi_{L}^{\beta}(w \bar{w}, \cdot) \\
& =\sum_{\substack{i m r \gamma \sigma \\
\bar{i} \bar{m} \bar{r} \bar{\sigma} \bar{\sigma}}}\left\{\sum_{\substack{k \mu \nu \\
k \bar{\mu} \bar{\nu}}} \widetilde{C}(j \bar{j} \alpha)_{i k \mu}^{i \bar{k} \bar{\mu}} \tilde{C}(l \bar{l} \beta)_{k m \nu}^{\bar{k} \bar{m} \bar{\nu}} F_{i j k l m r}^{\mu \nu \gamma \sigma} \bar{F}_{i \bar{j} \bar{k} l \bar{m} \bar{r}}^{\bar{\mu} \bar{v} \bar{\gamma} \bar{r}}\right\} \\
& \cdot\left[\varphi_{i r m}^{\gamma}(z, w ; \sigma, \cdot, \cdot)\right]\left[\varphi_{i \bar{r} \bar{m}}^{\bar{\gamma}}(\bar{z}, \bar{w} ; \bar{\sigma}, \cdot, \cdot)\right] \text {. }
\end{aligned}
$$


If we now remember the definition of the chiral fusion matrix in terms of the braid matrix, i.e. Eq. (5.9), and if we apply (8.14), what we end up with is

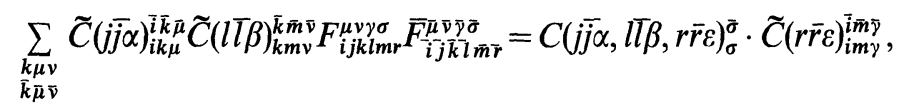

where the structure constant of the operator product expansion (OPE) is given by

$$
C(j \overline{j \alpha}, l \bar{l} \beta, r \bar{r} \varepsilon)_{\sigma}^{\bar{\sigma}}=\widetilde{C}(\overline{j j \alpha})_{r l \sigma}^{\bar{r} \bar{l} \bar{\sigma}} \cdot \frac{\widetilde{C}(l \bar{l} \beta)_{l 1}^{\bar{l} \overline{1}}}{\widetilde{C}(r \bar{r} \varepsilon)_{r 1}^{\bar{r} 1}} .
$$

The OPE thus has the form, $\varepsilon \equiv \varepsilon(r, \tilde{r})$,

$$
\begin{aligned}
& \phi_{J}^{\alpha}(z \bar{z}, \cdot) \phi_{L}^{\beta}(w \bar{w}, \cdot)=\sum_{\substack{r \sigma \\
\bar{r} \bar{\sigma}}} C(\overline{j j \alpha}, l \pi \beta, r \bar{r} \varepsilon)_{\sigma}^{\bar{\sigma}} \\
& \cdot \sum_{N, \bar{N}} \phi_{r \bar{r}}^{\varepsilon}\left(\frac{z+w}{2}, \frac{\bar{z}+\bar{w}}{2}, \eta_{r}^{N}, \eta_{\bar{r}}^{\bar{N}}\right) \cdot\left\langle\eta_{r}^{N}, \varphi_{r j l}^{\sigma}\left(\frac{z-w}{2}, \cdot\right)\right. \\
& \left.\cdot \varphi_{l l 1}\left(\frac{w-z}{2}, \cdot\right) \Omega\right\rangle \cdot\left\langle\eta_{\bar{r}}^{\bar{N}}, \varphi_{\bar{r} \bar{j} l}^{\bar{\sigma}}\left(\frac{\bar{z}-\bar{w}}{2}, \cdot\right) \varphi_{\bar{l} \bar{l} \overline{1}}\left(\frac{\bar{w}-\bar{z}}{2}, \cdot\right) \Omega\right\rangle .
\end{aligned}
$$

Equation (8.14) also provides us with the symmetry

$$
C(\overline{j \alpha} \alpha, l \bar{l} \beta, r \bar{r} \varepsilon)_{\sigma}^{\bar{\sigma}}=\sum_{\varrho \bar{\varrho}} R^{+}(i l j 1)_{j \varrho}^{l \sigma} \bar{R}^{-}(\overline{i l j 1})_{j \bar{\varrho}}^{\bar{l} \bar{\sigma}} C(l \bar{l} \beta, j \overline{j \alpha}, r \bar{r} \varepsilon)_{\varrho}^{\bar{\varrho}} .
$$

In order to derive all the symmetries of the OPE coefficients it is quite useful to state the assumption

$$
\phi_{J}^{\alpha} \neq 0 \Rightarrow \widetilde{C}(j \overline{j \alpha})_{1}^{\overline{1} \bar{j}^{*}} \neq 0 .
$$

In analogy to (8.14) we thus get

$$
\begin{aligned}
& \widetilde{C}\left(l^{*} T^{*} \beta\right)_{1 l}^{\overline{1} \bar{l}} \widetilde{C}\left(j^{*} \bar{j}^{*} \alpha\right)_{l m \nu}^{\bar{l} \bar{m} \bar{\nu}} \\
& \quad=\sum_{\gamma \bar{\gamma}} R^{+}\left(1 j^{*} l^{*} m\right)_{j \gamma}^{l v} \bar{R}^{-}\left(\overline{1} \bar{j}^{*} \bar{l}^{*} \bar{m}\right)_{j \bar{\gamma}}^{\bar{l} \bar{\nu}} \widetilde{C}\left(j^{*} \bar{j}^{*} \alpha\right)_{1 j}^{\overline{1} \bar{j}} \widetilde{C}\left(l^{*} \bar{l}^{*} \beta\right)_{j m \gamma}^{\bar{j} \bar{m} \bar{\gamma}},
\end{aligned}
$$

which links $\widetilde{C}\left(j^{*} \bar{j}^{*} \alpha\right)_{l m}^{\bar{l} \bar{m}}$. and $\widetilde{C}\left(l^{*} T^{*} \beta\right)_{j m}^{\bar{j} \bar{m}}$. Repeating those steps which led to (8.18), but now using (8.20) instead of (8.14), gives the remaining symmetry relations of the OPE coefficients. For example,

$$
\begin{aligned}
C\left(j^{*} \bar{j}^{*} \alpha^{*}, l \bar{l} \beta, r^{*} \bar{r}^{*} \varepsilon^{*}\right)_{\sigma}^{\bar{\sigma}}= & \sum_{\gamma \bar{\gamma}} R^{+}\left(1 j^{*} r l\right)_{j \gamma}^{r^{*} \sigma} \bar{R}^{-}\left(1 \bar{j}^{*} \bar{r} \bar{l}\right)_{j \bar{\gamma}}^{r^{*} \bar{\sigma}} \\
& \cdot \frac{\widetilde{C}\left(j^{*} \bar{j}^{*} \alpha^{*}\right)_{1 j}^{\overline{1} \bar{j}} \widetilde{C}(\overline{j \alpha})_{j 1}^{\bar{j} \overline{1}}}{\widetilde{C}(r \bar{r} \varepsilon)_{1 r^{*}}^{\overline{1} \bar{r}^{*}} \widetilde{C}\left(r^{*} \bar{r}^{*} \varepsilon^{*}\right)_{r^{*} 1}^{\bar{r}^{*} \overline{1}}} \\
& \cdot C(r \bar{r} \varepsilon, l \bar{l} \beta, j \overline{j \alpha})_{\gamma}^{\bar{\gamma}} .
\end{aligned}
$$

We summarize our results in

Proposition 26. On the set of local fields there is an operator product expansion, written down in (8.17). The OPE coefficients $C(j \overline{j \alpha}, l \bar{l} \beta, r \bar{r} \varepsilon)_{\sigma}^{\bar{\sigma}}$ enjoy the symmetries (8.18) and (8.21). 
If we restrict our attention (momentarily) to the case $j=j^{*}, \bar{j}=\bar{j}^{*}$, then it is easy to come up with the constraint

$$
\left(H_{j}-H_{\bar{j}}\right) \in \mathbb{Z} \text {. }
$$

The validity of (8.23) can be verified using (8.10), (8.13), and (8.19).

The local fields should be independent of normalization of the chiral vertices. The definition (8.1) then implies that under a gauge transformation

$$
\begin{aligned}
& \varphi_{i j k}^{\mu}(z, \cdot) \mapsto \lambda_{i j k}^{\mu} \cdot \varphi_{i j k}^{\mu}(z, \cdot), \\
& \varphi_{i \bar{j} \bar{k}}^{\bar{\mu}}(\bar{z}, \cdot) \mapsto \lambda_{i j \bar{k}}^{\bar{\mu}} \cdot \varphi_{i \bar{i} \bar{k}}^{\bar{\mu}}(\bar{z}, \cdot), \\
& \tilde{C}(\overline{j j \alpha})_{i k \mu}^{\bar{i} \bar{k} \bar{\mu}} \mapsto \frac{1}{\lambda_{i j k}^{\mu} \cdot \lambda_{i j \bar{k}}^{\bar{\mu}}} \cdot \widetilde{C}(j \overline{j \alpha})_{i k \mu}^{\bar{i} \bar{k} \bar{\mu}} .
\end{aligned}
$$

The formula (8.16) clearly shows that the OPE coefficient is gauge variant. Notice also that (8.16) was derived in the gauge (3.17). We can easily overcome this weakness by multiplying the right-hand side of (8.16) with normalization constants of the participating chiral vertices. For example, $\widetilde{C}(l \bar{l} \beta)_{l 1}^{\bar{l} \overline{1}}$ would be turned into $\left(\widetilde{C}(l \bar{l} \beta)_{l 1}^{\bar{l} \overline{1}} \cdot v_{l l 1} \cdot \bar{v}_{\bar{l} \overline{1} \overline{1}}\right)$, and so on.

The point is now that in simple circumstances the symmetries (8.18) and (8.21), combined with (8.10), allow us to compute the OPE coefficients explicitly. This happens if we consider chiral theories with $N_{i j k} \in\{0,1\}, N_{\bar{i} j \bar{k}} \in\{0,1\}$, and if $j=j^{*}$, $\forall j \in \mathscr{L}$, and $\bar{j}=\bar{j}^{*}, \forall \bar{j} \in \overline{\mathscr{L}}$. We look for a solution of (8.10) which obeys

a) per index pair $(j, \bar{j})$ there is at most one local field;

b) given $j$, at most one $\bar{j}$ is used to construct a local field.

To simplify things we normalize the local fields by requiring

$$
\left(\widetilde{C}(\bar{j})_{1 j}^{\overline{1}} \bar{j}_{1 j j} \bar{v}_{\overline{1} \bar{j} \bar{j}}\right) \cdot\left(\widetilde{C}(j \bar{j})_{j 1}^{\bar{j} \overline{1}} v_{j j 1} \bar{v}_{\bar{j} \bar{j} \overline{1}}\right)=1 .
$$

(This normalization is equivalent to the conventional normalization of primary fields in the case where $\mathscr{A} \simeq \overline{\mathscr{A}}=$ Virasoro algebra. For, in this special case, and if $\xi_{j}$ and $\xi_{\bar{j}}$ are the normed primary vectors of $\mathscr{H}_{j}$ and $\mathscr{H}_{\hat{j}}$, respectively, one usually requires $\left\langle\Omega, \phi_{j \bar{j}}\left(z \bar{z}, \xi_{j}, \xi_{\bar{j}}\right) \phi_{j \bar{j}}\left(w \bar{w}, \xi_{j}, \xi_{\bar{j}}\right) \Omega\right\rangle=1 \cdot(z-w)^{-2 H_{j}}(\bar{z}-\bar{w})^{-2 H_{\bar{j}}}$. If we define $v_{1 j j}:=\left\langle\Omega, \varphi_{1 j j}\left(1, \xi_{j}\right) \xi_{j}\right\rangle$, this normalization of the local fields leads to (8.26).)

Rewriting (8.10) by pulling $R^{+}$to the left-hand side, and setting $p=1, \bar{p}=\overline{1}$, gives

$$
R^{-}(i i j j)_{1}^{k} \cdot \widetilde{C}(i \bar{i})_{i 1}^{\bar{i} \overline{1}} \widetilde{C}(j \bar{j})_{1 j}^{\overline{1} \bar{j}}=\bar{R}^{-}(\overline{i j i j})_{\bar{k}}^{\overline{1}} \widetilde{C}(j \bar{j})_{i k}^{\bar{i} \bar{k}} \widetilde{C}(i \bar{i})_{k j}^{\bar{k}} \bar{j}
$$

Upon using the symmetries (8.21) and (8.18), and remembering that $\left(H_{j}-H_{\vec{j}}\right) \in \mathbb{Z}$, the definition of the OPE coefficients, (8.16), and the normalization (8.26) yield the gauge invariant OPE coefficients

$$
(C(i \bar{i}, j \bar{j}, k \bar{k}))^{2}=\frac{R^{-}(i i j j)_{1}^{k}}{\bar{R}^{-}(\overline{i j i j})_{\bar{k}}^{1}} \cdot v,
$$

where $v$ stands for a quotient of normalization constants $v_{i j k}, v_{i i 1}, \ldots$, such as to render the right-hand side of (8.27) invariant under rescalings of the chiral vertices.

For the left-right-symmetric minimal models the structure constants of the OPE have been calculated explicitly by Dotsenko and Fateev [16] by investigat- 
ing the monodromy behaviour of the 4-point correlation functions. Later on, Eq. (8.27) was used in [6] to rederive these results in a way which, in our understanding, is conceptually preferable.

Using results of Ref. [16], Fateev and Zamolodchikov [18] computed the structure constants for the left-right-symmetric $s \hat{u}_{k}(2)$-theories. rules:

In Sect. 7.1, link invariants have been constructed upon using the following

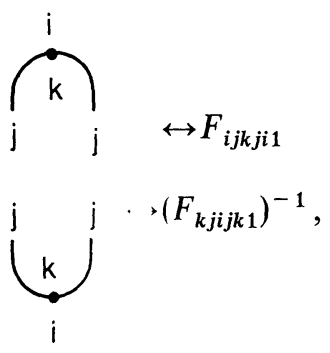

where we assumed $v_{m 1 m}=1, j=j^{*}$, and $N_{i j k} \in\{0,1\}$. This seems to be in rather sharp contrast to the rules which we formulated (for left-right-symmetric minimal models) in the second Ref. of [6]:

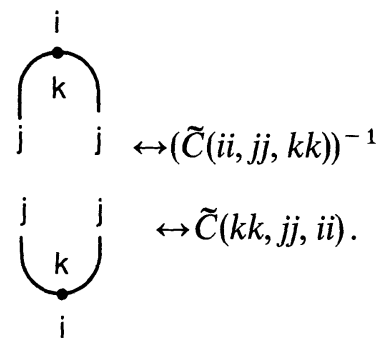

Let us explain why (8.29) and (8.28) are equivalent. By definition, the rule (8.29) applies to left-right-symmetric theories with $v_{m 1 m}=1, j=j^{*}, N_{i j k} \in\{0,1\}$, and $\widetilde{C}(i \bar{i}, j \bar{j}, k \bar{k})=\delta_{\overline{i i}} \delta_{\bar{j} j} \delta_{\bar{k} k} \widetilde{C}(i i, j j, k k)$, and it is valid in that gauge for the braid matrices in which $R^{+}(a b c d)_{e f}=R^{+}(d c b a)_{e f}$, which, by construction, exists at least for the minimal models [6]. In such a left-right-symmetric theory, the fusion coefficients $\widetilde{C}(i i, j j, k k)$ can be interpreted to serve the purpose of projecting $\mathscr{H}_{1}$ out of $\mathscr{H}_{j} \otimes \mathscr{H}_{j}$, which is also the purpose of $F_{i j k j i 1}$. This is the representation theoretic motivation to iterate the braid-fusion equation (5.15) with $r=1$ and $v_{a 1 a}=1$, which yields

$$
R^{+}(i j l m)_{k p} F_{i l p l i 1} F_{p j m j p 1}=R^{+}(i l j m)_{p k} F_{i j k j i 1} F_{k l m l k 1} .
$$

Comparing (8.30) with the locality equation (8.10), taking into account the symmetry of $R$, shows that a solution is

$$
\widetilde{C}(i i, j j, k k)=\left(F_{i j k j i 1}\right)^{-1} .
$$

There does not seem to exist a general method to deduce the value of the central extensions of the chiral algebras $\mathscr{A}$ and $\overline{\mathscr{A}}$, once one is given a set of $R$ - and $\bar{R}$-matrices which satisfy the polynomial Eqs. (4.19)-(4.21), (5.13)-(5.15) and the locality Eq. (8.10). However, if we make the additional assumption that the chiral 
field theories $(\overline{\mathscr{A}}, \overline{\mathscr{L}}, \overline{\mathscr{R}})$ and $(\mathscr{A}, \mathscr{L}, \mathscr{R})$ are consistent also on the torus, then it is shown in Ref. [4] that the representation of the mapping class group on the characters $\operatorname{Tr}_{j}\left(q^{L_{0}-c / 24}\right), j \in \mathscr{L}$, relates the conformal dimensions $H_{j}, j \in \mathscr{L}$, and the central extension, $c$, of the holomorphic Virasoro algebra. In particular, $c$ is a rational number, and the method just sketched allows for a determination of $c$ $\bmod 4$.

If we stick to theories on $S^{2}$, then at least if $\mathscr{A}=$ Virasoro algebra the knowledge of the 4-point block $\left\langle\xi_{i}, \varphi_{i i 1}\left(\xi_{i}, z\right) \varphi_{1 j j^{*}}\left(\xi_{j}, w\right) \xi_{j^{*}}\right\rangle$ is sufficient to compute the value of $c\left(\xi_{k}\right.$ is the unique primary vector in $\left.\mathscr{H}_{k}\right)$. Indeed, if we write $\left\langle\xi_{i}, \varphi_{i i 1}\left(\xi_{i}, z\right) \Omega\right\rangle=v_{i i 1}:=1$, and if $\left\langle\Omega, \varphi_{1 j j^{*}}\left(\xi_{j}, w\right) \xi_{j^{*}}\right\rangle:=w^{-2 H_{j}}$, assuming $H_{j}=H_{j^{*}}$, then the above 4-point block equals $w^{-2 H_{j}}\left(1-\frac{2}{c} H_{i} H_{j}\left(\frac{w}{z}\right)^{2}+O\left(\left(\frac{w}{z}\right)^{3}\right)\right)$.

Therefore, knowing the leading terms of the expansion of the 4-point block as $z \rightarrow \infty$ and $-\pi<\arg w<\pi$ gives immediately the value of $c$.

\section{Reconstruction of Chiral Field Theories from Quantum Groups}

The logic developed so far is: given suitable representations of a chiral algebra whose vertices transform under a linear representation of the braid group when being continued analytically, what can be said about the structure of the chiral field theory? Specifically, what kind of algebraic relations are obeyed by $R$, and can these relations be deduced from the representation theory of some quantum group (via a generalized Vertex-SOS transformation)?

But we may also reverse our point of view: We assume to be given a family of $R$-matrices which come, for example, from the representation theory of a quantum group, satisfying the algebraic equations of Sects. 4 and 5. Does there exist a chiral algebra and corresponding chiral vertices which reproduce the given $R$-matrices?

The answer, a solution of a generalized Riemann-Hilbert problem, is not known in general. However, there are some hints in the mathematical literature [17] that trying to solve this problem may not be a completely hopeless task. In fact, if the monodromy matrices $\mathrm{M}^{+}(\mathrm{ijlm})$ are sufficiently close to the identity then, according to Ref. [17], there is at least one (trivial) flat vector bundle over $\mathbb{C}^{n} \backslash\left\{\mathbf{z} \mid z_{i}=z_{j}\right.$, for some $\left.i \neq j\right\}$ whose monodromies are the given $M^{ \pm}$. Also, the connection 1-form $\Omega$ can be chosen to be $\Omega=\sum_{1 \leqq i<j \leqq n} d\left(\log \left(z_{i}-z_{j}\right)\right) \cdot \Omega_{i j}$, where $\Omega_{i j}$ are z-independent $\mathbb{C}$-number matrices.

Unfortunately, it is not obvious at all whether this candidate theory is satisfactory in the sense that the "conformal blocks" (i.e. the solutions to the parallel transport equation) have the required conformal properties; plus additional transformation properties which would make them transform under the action of a chiral algebra $\mathscr{A}$ which could be associated to the given monodromy matrices. For example, assume that there is a horizontal section $\vec{f}(\mathbf{z})$ which transforms covariantly under Möbius transformations $z_{i} \mapsto w_{i}\left(z_{i}\right): \prod_{i=1}^{n}\left(\frac{d w_{i}}{d z_{i}}\right)^{h_{i}} \vec{f}(\mathbf{w})$ $=\vec{f}(\mathbf{z})$. If we apply the parallel transport equation on this transformation law we find that a) translations and dilatations give no constraints at all, b) infinitesimal 
special conformal transformations are consistent with the form of the connection $\Omega$ iff, for each $i, 1 \leqq i \leqq n,\left(\sum_{j \neq i} \Omega_{i j}\right) \vec{f}(\mathbf{z})=-2 h_{i} \cdot \vec{f}(\mathbf{z})$. Here we defined, for $i>j$,
$\Omega_{i j}:=\Omega_{j i}$.

\section{References}

1. Felder, G., Fröhlich, J., Keller, G.: On the structure of unitary conformal field theory I: Existence of conformal blocks, ETH-preprint November 1988

2. Zamolodchikov, A.B.: Infinite additional symmetries in two-dimensional conformal quantum field theory. Theor. Math. Phys. 65, 1205 (1986)

3. Verlinde, E.: Fusion rules and modular transformations in $2 D$ conformal field theory. Nucl. Phys. B 300, 360 (1988)

4. Vafa, C.: Toward Classification of conformal field theories. Harvard preprint HUTP-88/A011

5. Moore, G., Seiberg, N.: Polynomial equations for rational conformal field theories. Phys. Lett. B 212, 451 (1988);

Naturality in conformal field theory. Nucl. Phys. B 313, 16 (1989);

Classical and quantum conformal field theory. IAS preprint IASSNS-HEP-88/39

6. Felder, G.: BRST approach to minimal models, ETH-preprint August 1988;

Felder, G., Fröhlich, J., Keller, G.: Braid matrices and structure constants for minimal conformal models. IAS preprint 1989

7. Knizhnik, V.G., Zamolodchikov, A.B.: Current algebra and Wess-Zumino model in two dimensions. Nucl. Phys. B 247, 83 (1984);

Gepner, D., Witten, E.: String theory on group manifolds. Nucl. Phys. B 278, 493 (1986)

8. Fröhlich, J.: Statistics of fields, the Yang-Baxter-Equation and the theory of knots and links, Cargèse Lectures 1987. 't Hooft, G. et al. (ed.). New York: Plenum Press 1988

Statistics and monodromy in two-dimensional quantum field theory, in "Differential geometric methods in theoretical physics." Bleuler, K., Werner M. (eds.). Dordrecht, Boston, London: Kluwer 1988

9. Tsuchiya, A., Kanie, Y.: Vertex operators in the conformal field theory of $\mathbb{P}^{1}$ and monodromy representations of the braid group. Lett. Math. Phys. 13, 303 (1987)

10. Birman, J.: Braids, links and mapping class groups. Ann. Math. Studies, vol. 82. Princeton, NJ: Princeton University Press 1974

11. Goodman, F., de la Harpe, P., Jones, V.: Dynkin diagrams and towers of algebras. Preprint Université de Genève, June 1986

12. Fröhlich, J., King, C.: Two-dimensional conformal field theory and three-dimensional topology, preprint ETH-TH/89-9

13. Reshetikhin, N.Yu.: Quantized universal enveloping algebras, the Yang-Baxter equation and invariants of links I, II, LOMI preprint E-4-87, E-17-87

14. Jones, V.: Hecke algebra representations of braid groups and link polynomials. Ann. Math. 126, 335 (1987)

15. Cappelli, A., Itzykson, C., Zuber, J.B.: Modular invariant partition functions. Nucl. Phys. B 280, 445 (1987);

The A-D-E Classification of minimal and $A_{1}^{(1)}$ conformal invariant theories. Commun. Math. Phys. 113, 1 (1987)

16. Dotsenko, V.S., Fateev, V.A.: Conformal algebra and multipoint correlation functions in $2 D$ statistical models. Nucl. Phys. B 240, 312 (1984);

Four-point correlation functions and operator algebra in $2 D$ conformal invariant theories with central charge $\leqq 1$. Nucl. Phys. B 251, 691 (1985);

Operator algebra of two-dimensional conformal theories with central charge $\leqq 1$. Phys. Lett. B 154, 291 (1985)

17. Kohno, T.: Hecke algebra representations of braid groups and classical Yang-Baxter equations, preprint;

Linear representations of braid groups and classical Yang-Baxter equations, Contemp. Math., vol. 78 (1988), “Braids, Santa Cruz, 1986," 339 
18. Fateev, V.A., Zamolodchikov, A.B.: Operator algebra and correlation functions in the twodimensional $s u(2) \times s u(2)$ chiral Wess-Zumino model. Sov. J. Nucl. Phys. 43, 657 (1986)

19. Belavin, A.A., Polyakov, A.M., Zamolodchikov, A.B.: Infinite conformal symmetry in twodimensional quantum field theory. Nucl. Phys. B 241, 333 (1984)

20. Friedan, D., Qiu, Z., Shenker, S.: Details of the non-unitarity proof for highest weight representations of the Virasoro algebra. Commun. Math. Phys. 107, 535 (1986);

Goddard, P., Kent, A., Olive, D.: Commun. Math. Phys. 103, 105 (1986)

21. Kastor, D., Martinec, E., Qiu, Z.: Phys. Lett. 200B, 434 (1988);

Bagger, J., Nemeschansky, D., Yankielowicz, S.: Phys. Rev. Lett. 60, 389 (1988);

Bowcock, P., Goddard, P.: Nucl. Phys. B 305, 685 (1988)

22. Jimbo, M.: Lett. Math. Phys. 10, 63 (1985);

Drinfel'd, V.G.: Quantum groups, Proc. ICM 798 (1987)

23. Pasquier, V.: Commun. Math. Phys. 118, 355 (1988)

24. Rehren, K.-H.: Commun. Math. Phys. 116, 675 (1988);

Rehren, K.-H., Schroer, B.: Einstein Causality and Artin Braids, preprint, FU Berlin, 1988

25. Gervais, J.-L., Neveu, A.: Nucl. Phys. B 238, 125 (1984)

Communicated by A. Jaffe

Received July 3, 1989; in revised form December 4, 1989 
